\title{
Sacred Technologies: \\ The evolution of religious cognitive niche
}

\author{
By
}

David J. Murphy

A thesis submitted to the Victoria University of Wellington in fulfillment of the requirements for the degree of Master of Arts in Religious Studies

School of Art History, Classics and Religious Studies

Victoria University of Wellington

2009 


\section{ABSTRACT:}

Most cognitive studies of religion adopt a modular theory of cognition. The 'space' that is studied is often the 'space between the ears'. Culture and religion are viewed as by-products of more entrenched features of our brains. Although this 'Standard Model' explains many intuitive expressions of religious belief, it has trouble explaining (a) the variability of religious systems crossculturally (b) the uses of material culture (i.e. symbolic structures etc) in transmitting religious concepts. The following thesis presents a 'wideware mind' hypothesis for religious cognition. I urge that while our internal cognitive architecture is causally relevant to religious cognition, the material artefacts of culture must be viewed as cognitive properties in their own right. Hence any causal account of religious cognition must acknowledge the external features of minds and how our neurological resources interact with the artefacts of our world. 


\section{ACKNOWLEDGEMENTS}

The author wishes to express his sincere thanks and appreciation to my primary advisor Dr Joseph Bulbulia and for giving me the opportunity to be involved in this field - it has been a real privilege. Without your enthusiasm and support this thesis would have never been completed. Also, many thanks to my secondary advisor Dr Michael Radich, whose help and input on this thesis have been invaluable.

Credit is also due to Aliki Kalliabetsos, administrator at the Religious Studies Department - you're amazing (enough said). I would also like to thank the very gifted academic staff at the Religious Studies Department here at Victoria University. It has been a real pleasure to work in such a supportive and friendly environment.

Recognition also goes to Professor Kim Sterelny from the Philosophy Department at Victoria University. You're work and contribution to the field of Philosophy of Biology has greatly benefited this thesis - cheers.

Thanks also to the Culture and Cognition reading group at Victoria University. The enthusiasm from all who are involved has made it a remarkable forum for discussion and debate.

Lastly, my most special thanks go to my friends and family for their support during the writing of this work: my Mother, John Rogers, Erin Maaskant, Daniel Dowling and my wonderful partner Emily Joliffe. Thank you. 


\section{Table of Contents}

TITLE 1

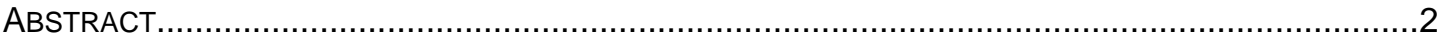

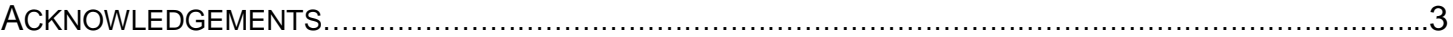

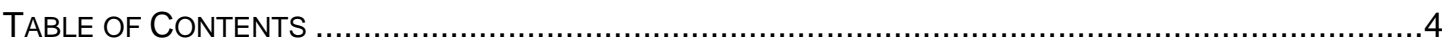

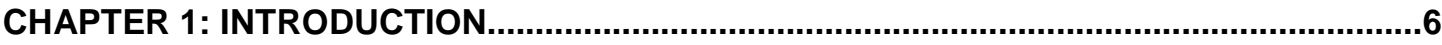

1.1.1. Religious Cognition and Material Culture .........................................................6

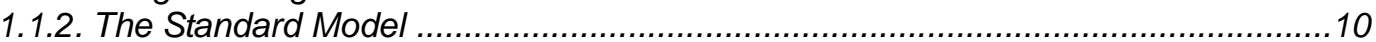

1.1.3. Problems with the Standard Model............................................................... 14

1.1.4. Minds and Artefacts........................................................................... 19

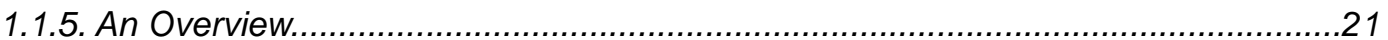

CHAPTER 2: EVOLUTIONARY PSYCHOLOGY: AN OVERVIEW......................................29

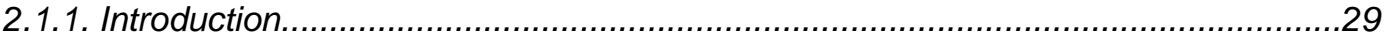

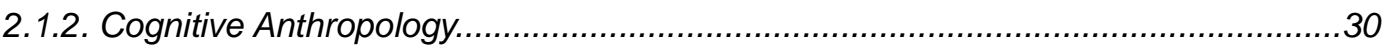

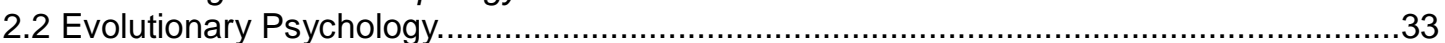

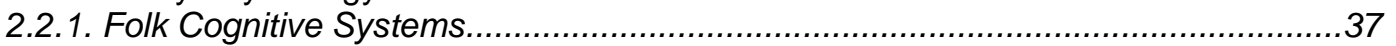

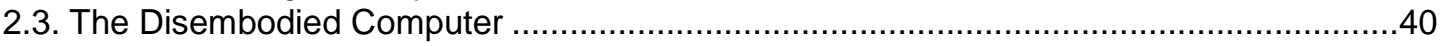

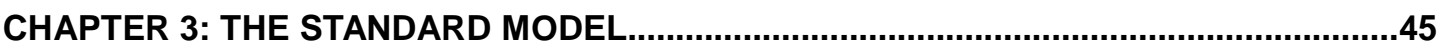

3.1. Implicit versus Explicit Religious Representations.....................................................46

3.2.1. Pascal Boyer: Religion as a cognitive by-product.......................................48

3.2.2. Minimally Counterintuitive Concepts......................................................50

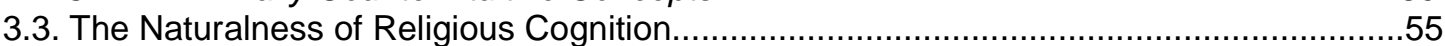

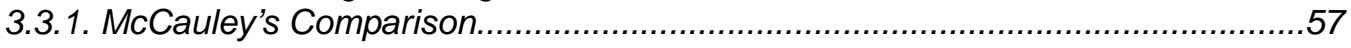

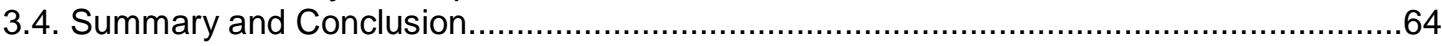

CHAPTER 4: CO-EVOLUTIONARY MODELS.............................................................67

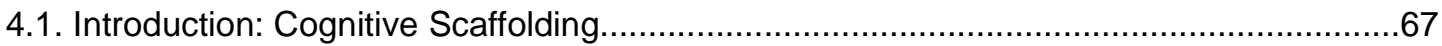

4.1.1. Dumb Brains, Smart Environments: Wideware Cognition....................................70

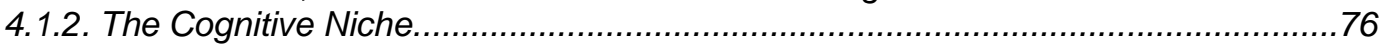

4.2. The Co-Evolution of Human Intelligence: Evidence from neuro-psychology.....................81

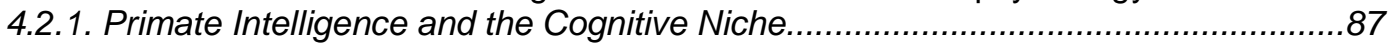

4.2.2. Towards a Co-Evolutionary model for Hominines................................................89

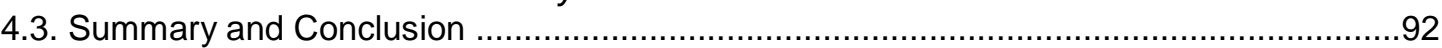

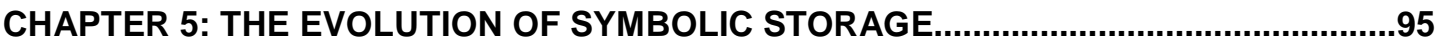

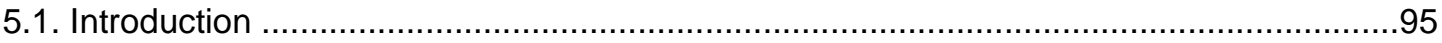

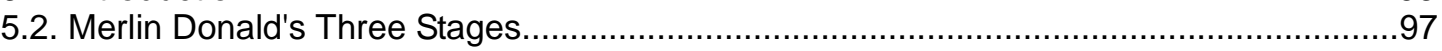

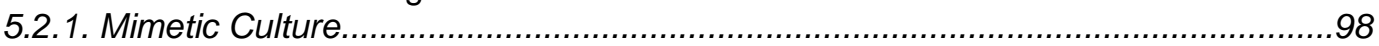

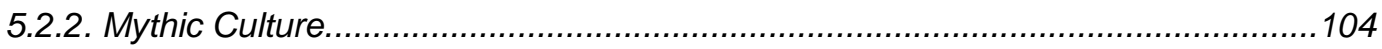

5.2.3. External Storage and Theoretical Culture …...................................................112

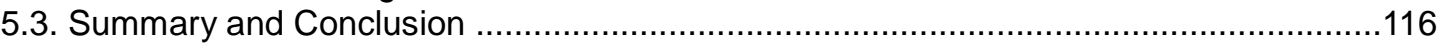

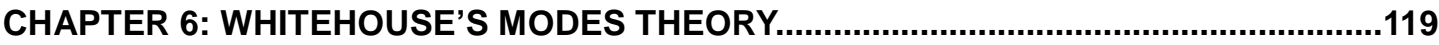

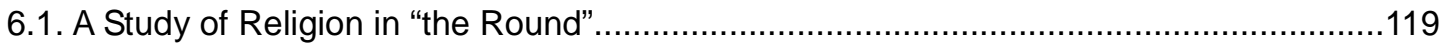

6.1.1. "Open" versus "Closed" Behavioural Programs...........................................123

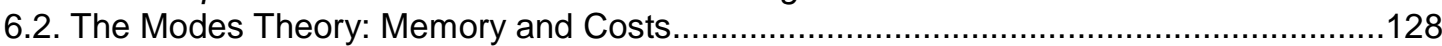

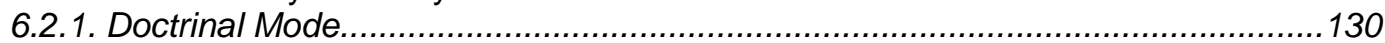

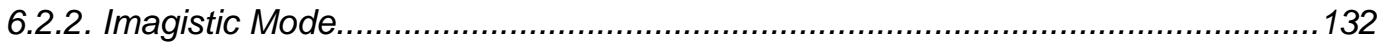

6.3. Socio-Ecological Engineering and The Modes Theory................................................134

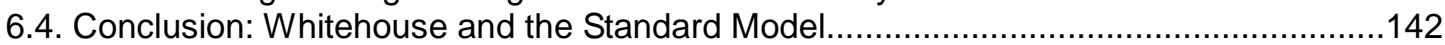

CHAPTER 7: TOWARDS A WIDEWARE MODEL OF RELIGIOUS COGNITION...............146

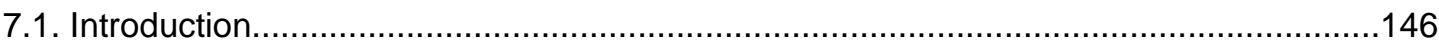

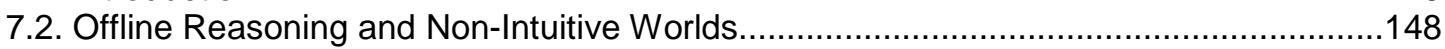

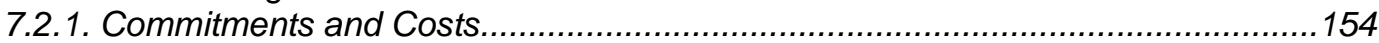

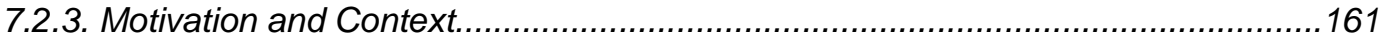


7.3. The Religious Cognitive Niche

7.3.1. Cognitive Archaeology and the Religious Niche.............................................173

7.3.2. The Emergence of Symbolic-Religious Behaviour...........................................183

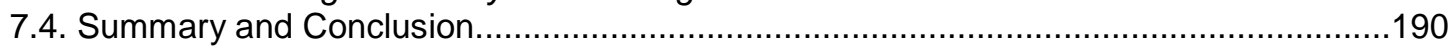

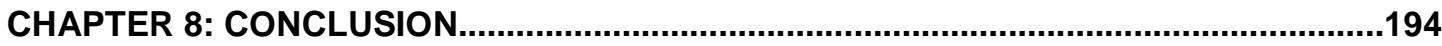

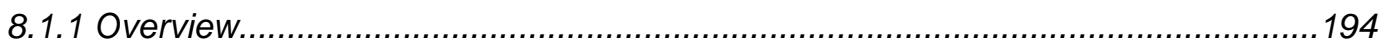

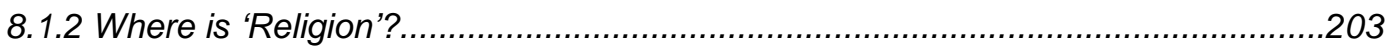

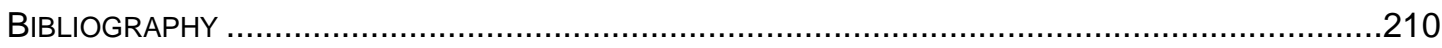




\section{Chapter 1: Introduction}

\subsubsection{Religious Cognition and Material Culture}

This thesis is about how our cultural practices and material artefacts function as a support for human cognitive processes. Humans operate within an environment whereby material culture enables both learning and problem-solving. Most of us employ pen and paper to record ideas, events or grocery lists; geographical maps provide direction, while arithmetic offers more efficient methods for tracking the quantity of units. Learning development, in conjunction with cultural devices, presents an abundance of solutions to everyday problems and constitutes the technological basis of humankind. Cultural environments - on this view - are comprised of properties consisting of artefacts and external support for cognitive development. Non-biological tools augment our biological limitations and make us smarter.

How is it that non-biological artefacts have evolved to support religious beliefs and activities? Many of the approaches within the cognitive science of religion (while being valuable to understanding the psychological motivations underlying religious behaviour), emphasizes the internal cognitive systems which form religious representations, while excluding the external properties which shape the religious mind. This thesis argues that internal cognitive systems 
are an insufficient explanation for the motivational salience of religious beliefs and activities. Instead I argue that a considerable portion of human cognitive expertise is dependent upon both material culture and the cooperative transmission of information. More specifically then, I will examine the intersection between human cognition and the cultural artefacts of religion.

'Religion' is a difficult term to define. Religious beliefs and activities can be broken down into a variety of parts consisting of rituals, supernatural representations, cosmologies, institutions, social norms (the list goes on). Space is limited, so this thesis does not seek to offer a rigorous definition of religion. However, I will say that literature on religious cognition often neglects this point, focusing instead upon just a single feature of religious behaviour without acknowledging the larger network of traits which constitute (what we often call) 'religion'. When using the term religion then, it is difficult to discern the focus of analysis without an operational definition. For the sake of clarity however this thesis will keep the 'question of definition' relatively open. Indeed, the problem of classification - as I will show later remains a relevant obstacle for most models within cognitive science of religion.

What I will argue however is that the various facets which constitute 'religion' are in large part the product of a unique coevolutionary process between culture and human cognition (if these 
two domains can indeed be discussed separately). Other models of religious cognition however have focused on internal psychological traits such as 'evolved predispositions' for religion, or 'cognitive biases' to religious ideas. Alternatively, I argue that the concepts and activities that motivate religious behaviour are especially difficult to develop without the evolution and use of material culture. Sacred cosmologies, rich theological bodies of knowledge, and elaborate rituals are aspects of religious behaviour which employ symbolic representations. Much like complex mathematical knowledge is difficult to comprehend without the use of symbols and artefacts, so too is complex supernatural reasoning unlikely without the use of culturally evolved technologies (Day 2005). Ritual, material symbols and artefacts make these complex religious worlds cognitively manageable. It takes the right kind of artefacts to transmit religion, but it also takes the right kind of brain also, a cognitive architecture that is almost dependent upon developmental learning and culturally evolved tools.

The cultural developmental thesis is important because it recognises first that religious cognition is not a biologically evolved disposition. In other words, the brain is not well-suited for processing religious representations. Human cultures have constructed environments which support the transmission of religious representations. The point of interest here is that external properties which produce supernatural thinking and actions are a sought after 
commodity within societies. This thesis argues that humans have constructed these 'sacred technologies' for a reason: the complex conceptual reasoning associated with religious thought augment cognitive abilities. By engaging in the higher level thinking associated with abstract representations, humans have devised techniques to extend the boundaries of thought, while further developing what Merlin Donald has called a 'theoretic culture' (Donald 1991; 2001). Through the manipulation of symbols, Donald argues that humans have established a way of extending the boundaries of memory and thought. Yet complex representations - in turn - act back upon the manipulator in a kind of cognitive feedback loop. The development of external symbolic representations is but one aspect of a theoretic culture, as it takes a particular type of cultural intelligence to engage with these cultural devices.

It could be said then that the rich symbolic and theological dialogue associated with religious thought have augmented cognitive abilities in much the same way that mathematical symbols have enabled computation and problem-solving. The abstract significance consigned to sacred objects and locales, for instance, has enabled the development of a collective representational system of value. The intrinsic value assigned to sacred objects is an unusual cognitive ability, yet it is also a highly motivational force in cultures (Renfrew $2001 ; 2008)$. The symbolic value that individuals place upon sacred objects may not have any real utilitarian worth, but these public 
representations can be effective social organisers for regulating collective behaviour (e.g. such as in the case of collective ritual and doctrinal social norms based around sacred objects). An external symbolic system of value (i.e. the sacred) is but one of the representational abilities that has developed alongside religious reasoning. But the complexity of religious theology shows that this class of knowledge poses cognitive and computational demands. The high-informational load that constitutes religious beliefs and activities maybe without explanation - if only for its seemingly non-utilitarian corollaries - but it is something that will be examined further in this thesis.

\subsubsection{The Standard Model}

Firstly, I will present a critical evaluation of the methodologies employed with the cognitive science of religion, particularly what is sometimes called the Standard Model and the obvious strengths and limitations therein (for definition of Standard Model see Boyer 2005; see also Day 2005; Whitehouse 2005). Secondly, I will present some of the theoretical and methodological challenges Standard Model within the cognitive science of religion. By way of these departures, I wish to offer an alternative to this Standard Model that encompasses externalist models of human cognition (i.e. extended mind, hybrid mind and cognitive niche construction hypotheses). Religious reasoning is not just distinguished by neurological mechanisms, but 
by the parity between internal and external cognitive properties (Clark 1997; Clark and Chalmers 1997).

The Standard Model argues that religion, whatever its classification (and a fair classification has yet to be determined I believe), remains ubiquitous within cultures because our innate psychological systems are naturally susceptible to supernatural ideas. According to the Standard Model 'religion' appears to be a universal feature within human cultures. In addition, a premise upon which the cognitive sciences rest is the biological invariable that humans are endowed with the same evolved cognitive hardware. Thus, perhaps there exists some perennial feature of our mental faculty which predisposes us to religious ideas and activities. Indeed many within the cognitive science of religion - particularly within the Standard Model - regard the capacity to conceive of the supernatural as a 'habit of the mind' (McCauley 2000). Religion comes 'naturally' to us because we have the kinds of brains that we do as biological endowment ${ }^{1}$.

What sets the Standard Model apart from other methodologies within the cognitive science of religion is that: Firstly, it assumes that a number of our psychological systems are genetically endowed and

\footnotetext{
${ }^{1}$ For a good introduction to some of the theories and methods within the Standard Model of cognitive science of religion, refer to: Scott Atran's "In Gods We Trust: The Evolutionary Landscape of Religion" (Oxford University Press, New York, 2002); Justin Barrett's "Exploring the Natural Foundations of Religion," (in Trends in Cognitive Sciences, 4: 29-34, 2000); Pascal Boyer's "Religion explained: The evolutionary origins of religious thought" (Basic Books, 2001); E Thomas Lawson, and Robert McCauley's "Rethinking Religion: Connecting Cognition and Culture" (Cambridge University Press, 1990); Robert McCauley's 'The Naturalness of Religion and the Unnaturalness of Science' (in F.C.Keil and R.A.Wilson [eds], Explanation and Cognition, Cambridge University Press, Cambridge, pp. 61-85, 2000).
} 
for obvious reasons have been subject to evolutionary processes. For the Standard Model, then, religion is conspicuous only because it persists as a universal feature within human cultures. Consequently the Standard Model posits that are correlations between universal features of our cognitive architecture and universal features of culture. The regular features of our cultural milieu are thus the result of internal cognitive regularities. Yet despite this phenomenon religious representations are not necessarily assumed to be standard features (i.e. inbuilt neurological characteristics) of our evolved cognitive systems (Boyer 1994, 2001). Explicit religious representations (e.g. ghosts, goblins, faeries and the gods) are instead viewed as a natural by-product of the cognitive mechanisms that generate these concepts (Boyer 2001). Thus the same cognitive processors that deal with practical (adaptive) behaviour are also the same processors that deal with the gods.

Secondly, explicit representations (such as Buddha, Christ, demons, ghosts etc), aren't studied comparatively as is so common within the anthropological approaches to religion. Instead the focal point of study is the implicit motivations underpinning the cultural production of these religious representations. Hence an understanding of the cognitive systems that constrain religious/cultural properties remains central to the study of religious behaviour - despite the diversity of religions reflected in the ethnographic literature. Thus on the Standard Model view there 
appears to be present in human culture a limited catalogue of supernatural representations that conform to very specific set of regularities defined by features of our cognitive architecture (Boyer 2001):

"Religious notions are products of the supernatural imagination. To some extent, they owe their salience (likelihood of activation) and transmission potential to features that they share with other supernatural concepts, such as found in dreams, fantasy, folktales and legends. This might be why one finds recurrent templates in religion despite many variations between cultures" (Boyer 2003:119).

Thirdly the Standard Model argues that humans are susceptible to these supernatural concepts precisely because we come equipped with a specific type of cognitive system (Boyer 1994; 2001). We are religious because our brains are vulnerable to religious ideas. Researchers within the 'Standard Model' recognise this fact that human brains seem to be well suited to religious concepts.

Supernatural representations are therefore relatively user-friendly, requiring precious little processing requirements. Like language, religion is deemed a naturally re-occurring, pan-human trait (Barrett 2000; Bulbulia 2005). Certainly, it seems as if religion is a lot like language in that it is an intuitively easy thing to be competent with:

"Humans (especially pre-pubescent humans) readily acquire and use natural languages... By better 
understanding how the particulars of our languageprocessing systems handle information, we have been able to better understand why human languages take the forms that they do. Cognition informs and constrains linguistic expression. Analogously, many different mental tools inform and constrain religious expression" (Barrett 2007: 769)

Consequently defenders of the Standard Model argue that, like language, religion requires precious little cultural inputs to enable its expression. Religion then is constituted by "non-reflective habits of mind" (McCauley 2000:63). Thus in-order to think religiously all we have to do is to think "naturally" (Barrett 2000). Consequently if the cognitive sciences can identify certain native traits (i.e. innate behavioural dispositions) then it can also inform us of why religions look the way they do. Subsequently it can also inform us of why humans engage so readily in supernatural beliefs and practices.

\subsubsection{Problems with the Standard Model}

The Standard Model assumes that the cognitive apparatus that generates religious representations is context-independent. In other words, it views the mind as being isolated from the socio-ecological context into which it is embedded. Alternatively this thesis will demonstrate that religion is constituted by a distributed cognitive system comprised of not only minds, but bodies, nervous systems and the properties of material culture (Day 2004b, 2005; Whitehouse 
2004). Within a distributed cognitive model the emphasis is placed upon agents and the environment. Thus material artefacts shouldn't be viewed then as disconnected components, but as "cognitive properties in their own right" (Hutchins 1995b:266). Artefacts facilitate human cognitive expertise.

Thus religion is enabled by distributed cognition - by agents engaged with features of their environment. The integration of brains and environment might constitute what cognitive neuroscientist Merlin Donald calls 'hybrid intelligence' (Donald 1991; 2001). Alternatively we could view this engagement between artefacts and brains as a multi-modal interactive dynamic, or what Andy Clark (2001) calls a 'wideware' cognitive system (whereby the mind is 'extended' into our environment). Similarly I argue that human cognition is something more than individual cognitive processes. Consequently, religious artefacts are just as important to an understanding of the 'religious mind' than that of our internal cognitive architecture.

My second concern is with the Standard Model's assumptions about the motivational machinery that drives religious behavioural outputs. Hence within the Standard Model, intuitive inferences constrain religious representations first-and-foremost. However, Harvey Whitehouse and others observe that the socio-political arrangements associated with a religious belief system seem to be designed to override normal intuitive inferences (Whitehouse 2004; 
Bulbulia 2004; Day 2004a, 2004b; Sosis and Alcorta 2003). Though it appears to the Standard Model that religious representations are engaged with by agents with a relative ease (this is the user-friendly argument for religion), the literature seems to ignore the demands required by religion, particularly the cognitive and physical costs of membership.

Religious reasoning cannot merely be a 'habit of the mind'. Agents within a religious belief system expend a considerable level of time, energy and resources to ensure that a complex system of beliefs and practices is sustained. Our intuitions aren't guided by these costs. On the contrary, the cognitive and physical demands of religions are sometimes harrowing ordeals requiring time, resources and energy (intuitively I would prefer to eat rather than fast; or build a home rather than a temple). Theological complexity, particularly the costs in learning and transmitting religious knowledge, is but one of the many informational costs - notable only because a lot of religious information is not necessarily adaptive (i.e. if you compare the adaptive value of foraging techniques to the value of prayer). Consequently, a problem for the Standard Model then is offering an explanation for why societies perpetuate these non-intuitive demands.

There are indeed cognitive constraints that enable and affect the processing of religious information. Memory systems for instance limit 
the storage and transmission of information. However these systems are conjunct with features of a particular context. I will argue that artefacts and the developmental environments of a belief system should be viewed as the external cognitive resources which support religious reasoning. Religious intelligence is enabled by the niche environments which humans have constructed in order to support the transmission of their beliefs and practices. I argue that without the capacity to shape our developmental environments then the transmission of religious concepts would be improbable given the limits of our neurological resources.

This notion that human enterprises consist of modifying their environment to benefit cognitive development is not new. There is a popular impression that natural selection favors organisms that adapt to environmental pressures. This is undoubtedly true. However, organisms have also countered these pressures by modifying their environment to suit their own ends and this biological activity is called niche construction (Laland \& Odling-Smee 2000a). Earth worms regulate the chemical properties of soil, spiders spin webs and termites build mounds. There are many examples in the animal kingdom of organisms engaging in environmental modification. As ecological engineers, humans have surpassed other organisms for their innovativeness in developing an environment which not only provides them with a fitness advantage, but makes them smarter too. A pen and parchment, in conjunction with one another, function as 
cognitive tools for both communication and memory. A message may be passed on; or a record of deeds kept. In both cases, individual mental processes access and interact with cultural artefacts which enable a wider cognitive interface.

So we inhabit a system of distributed knowledge - a niche constituted by information and tools for managing day-to-day living. Within the distributed cognition model, aspects of human intelligence aren't limited to just the individual's internal cognitive processes (Hutchins 1995a; 1995b). Instead, knowledge is 'distributed' by the attributing of cognitive properties to tools, artefacts and other individuals in the environment. Thus knowledge is stored and shared within human environments. Agents learn and teach others' how to utilize cognitive tools for living. We are creatures of technology, because we are also highly pro-social as well and without the cooperation associated with human sociality this mode of transmission would be impossible (Sterelny 2007).

\subsubsection{Minds and Artefacts}

Religious information is not just the product of basic brain activity. Instead religious information is actively external within the cultural environment. By this I mean that information is both external and actively utilized in the transmission of beliefs and practices. As Matthew Day (2004b) points out: if we view cognition as purely 
'internal' phenomena we subsequently overlook a rather crucial feature to the study of the religious mind, that the rich array of religious belief systems benefit significantly from external/cultural material properties also. Thus if we can additionally embrace the notion that brains are extended into the world, we must also perhaps ask why and how minds work in conjunction with material artefacts. The distinguishing of minds from the cultural network where minds take cognizance can be tricky especially if only fragmentary explanations can be offered in which differing levels of organization within a biological system take precedence over others. As Karola Stotz and Paul Griffiths (2000) observes:

"Part of the rationale of the traditional idea of human nature was to isolate features that do not depend on culture. These 'biological' features represent our true nature - the naked ape stripped of its cultural clothes. It seems to us that this traditional project is as misguided as seeking to investigate the true nature of an ant by removing the distorting influence of the nest! Human beings and their cultures have co-evolved as surely as ants and hives or dogs and packs" (Griffiths and Stotz 2000:4445).

Similarly, the cognitive science of religion should seek explanations for religious behaviour at differing levels of organization. However, this includes recognising: a) cognitive flexibility in human development; and b) how artefacts extend religious reasoning and thought. In order to gain a more comprehensive view of religious 
cognition we additionally have to take into account why it is that "many species-typical features of human psychology may depend critically on stably replicated features of human culture" (Griffiths and Gray 1994:302). Thus, a comprehensive picture requires a multi-level explanation for religious cognition.

Harvey Whitehouse argues that in order to establish more robust explanations within the cognitive science of religion we must first be able to carve the "... subject matter at the joints" thus laying bare "the mechanisms that shape religious thinking" (Whitehouse 2004:15). However, the causal explanation offered by the Standard Model depends upon demarcating features that are more relevant than others. For instance, the Standard Model highlights brains over socioecological factors. However Whitehouse proposes that there is still room for a more "rounded" picture within the cognitive sciences incorporating a variety of characteristics - both internal and external which shape the religious mind.

\subsubsection{An Overview:}

In the next chapter I will review some of the more common approaches within the field of Evolutionary Psychology. It is within these theories and methods that the Standard Model has established itself within the cognitive sciences. The evolutionary and biological sciences once applied to the cognitive sciences has made a 
significant impact on the study of religion within the Standard Model and set out a foundation for a research agenda underpinned by what is often dubbed 'nativist' thesis, sometimes also called the massive modularity hypothesis (MMH) (Samuels 1998; Sterelny 2003). Psychological nativism holds that certain behavioural traits or skills are inbuilt as biological endowments.

The 'modularity' thesis holds that there are specialised cognitive and neurological resources devoted to dealing with certain tasks. For instance, cognitive psychologists have identified certain behavioural characteristics that develop in children almost independently of environmental influences such as an innate understanding of physical dynamics such as solidity (Spelke, 1990; 1998), gravitational dynamics such as weight (Schilling \& Clifton 1998) and language-use (Chomsky 1988). Evolutionary Psychology (a particular branch of psychological nativism) has extrapolated this observation in support of a model highlighting evolved psychological traits.

Following an outline of some of the conceptual approaches to an understanding of human cognition within Evolutionary Psychology, the third chapter will review the Standard Model. This will begin with an overview of the theories and methods offered by cognitive anthropologist Pascal Boyer who has been at the forefront within the field of religion and the cognitive sciences. Boyer is notable as the chief purveyor of what is now called the Standard Model. Though 
Boyer recognises that religious behaviour is both a pan-human quality and naturally acquired by individuals, he's convinced that religion is not in our genes per se. We are not biologically endowed with an identifiable religious trait, nor is religious cognition an evolved adaptation. Instead our brains are naturally predisposed - or susceptible - to religious ideas. Thus while being natural to us, religion is certainly not a natural feature of our biological make-up according to Boyer. In addition, I will review Robert McCauley's definition of naturalness and the "naturalness of religion" hypothesis, which argues that religious beliefs and practices are an intuitive behavioural program only because our brains are well suited for accepting 'religious' explanations for worldly phenomena.

The fourth chapter will outline some criticisms against the orthodox conception of Evolutionary Psychology. In particular, this chapter will focus on Merlin Donald's argument against Evolutionary Psychology and its theoretical shortcomings. Here Donald argues that we shouldn't view the neuro-cognitive equipment as a system comprised of specialised domains, instead our brains are far more integrated. Above all our cognitive system should be viewed as being constituted by neurological properties and external/cultural properties also. In addition, the fourth chapter will review some of the relatively new evidence for a wideware model of mind (Clark 2001). It is here that I will present four models which share some commonalities with one another: hybrid mind (Donald 1991; 2001); distributed cognition 
(Hutchins 1995a; 1995b); extended mind (Clark 1997; Clark and Chalmers 1997); and cultural niche construction models (Laland and Odling-Smee 2000a, 2000b; Sterelny 2003). These models all share key conceptual similarities: they view the external environment as a causally relevant factor in the development of cognitive expertise. These models mutually support an understanding of cognition as being a product of internal (neurological) and external (cultural/socioecological) resources. The common features of these models constitute what Andy Clark (2001) has classified as a wideware understanding of cognition.

The fifth chapter introduces the concept of symbolic storage and the evolution for the capacity to extend into the environment symbolic representations in the form of material culture. It is here that Merlin Donald specifies within his hybrid mind model the triadic development of mimetic, mythic and technologically supported culture which has produced - within a co-evolutionary process - 'modern' human cognitive expertise. I will show that the cognitive niche is additionally constituted by symbolic properties which condition agents to their cultural context. Indeed if we view the symbolic world which agents inhabit as - what Chris Knight (1998) calls - a 'collectively perpetuated fiction', then we maybe able to conclude that cultural worldviews (particularly religious worldviews) are additionally shaped by learning development within this symbolic niche. The symbolic worlds in which individuals are embedded shape beliefs and 
practices, but in turn these activities perpetuate a shared context. Development and context thus conditions individuals to function within the reality of these symbolic worldviews.

The sixth chapter will concentrate primarily on Harvey Whitehouse's views. Whitehouse critiques the standard model's conception of the religious mind outside of the context into which agents are embedded. Whitehouse is also critical of the cognitive regularities that the standard model offers as being more significant to the production religious representations. He instead proposes that human religiosity is best explained as produced within a wider cognitive system: the socio-ecological milieu. While Whitehouse offers a cultural selectionist model within the transmission of religious representations, he nevertheless argues that human societies have created beliefs and practices that conform to either what Whitehouse calls imagistic or doctrinal modes of religiosity.

I conclude this chapter by explore some archaeological theories relevant to the emergence of human religiosity. Archaeological data will be a crucial element in the presentation of the theoretical material discussed regarding the wideware approach. The culturally evolved features of human socio-ecologies should be indicative of both the beliefs and activities of cultures, but they should also reveal how the modified socio-ecology influenced the habits of those individuals occupying the niche itself. There are many problems in considering 
this kind of evidence only because interpretations of the scarce data are disputed. But if both Whitehouse's model of human religiosity (and subsequently the wideware model) is to gain any foothold, then it is archaeology that is going to provide us with any clues as to why and how cultural practices and artefacts enable supernatural cognition.

After reviewing these alternatives to the Standard Model, I relate it to the theoretical models discussed in chapters four and five. An important task for my thesis will be identifying compatible features within these models in order to ensure their application. The theological worlds of religious agents are indeed comprised of symbolic properties, but these are by no means a consequence of religious beliefs and activities by the producer and product of a belief system. Religious beliefs and practices are an example of our cognitive capacity to construct complex, interwoven symbolic environments. The relationship between minds and symbolic properties - this cognitive interdependence - thus determines a religious context whereby a system of beliefs is transmitted. The religious niche is arranged in such a way as to pass on information that would not normally be available without the use of external cognitive devices such as material symbols. This understanding of the religious mind being extended into the environment and confluent with the cultural properties of the religious requires a novel approach 
to the evolutionary study of religion - one that is lost on the Standard Model.

Following this I review Steven Mithen's extended mind approach to archaeology (Mithen 1996a, 1996b; 2001). It is in this section of the final chapter that some of the approaches associated with coevolution and the wideware model are illustrated within the study of material culture and the development human cognitive expertise. Again archaeology remains important to the co-evolutionary dynamics that have led to modern human intelligence, because material culture has played a role in how humans think and behave. We not only depend upon the artefacts around us for survival, but artefacts also support our intellectual capabilities also. Mithen states that material culture has acted and currently acts as a cognitive "anchor" enabling a robust interface between a variety of domains, including internal cognitive processes and external cognitive properties. Religious material culture in particular, Mithen argues, has enabled the transmission of supernatural concepts. Supernatural ideas, he suggests, are far too cognitively demanding without the use of symbolic artefacts.

The religious niche exists as an environment comprised of factors encompassing the socio-ecological (e.g. demographic, institutional), and the technological (e.g. symbolic artefacts). Though niche environments do have a profound affect upon the organisms that 
inhabit them, humans are unique in that they modify their own niche environments to meet their purposes (Laland and Odling-Smee 2000a, 2000b). In turn, these environments are inherited by current and future generations who are considerably shaped by their adapted milieu. A cultural (or cognitive) niche significantly influences the beliefs and actions of the agents who inhabit them. If it can be shown that supernatural representations require the use of cultural technologies, then it demonstrates that humans have sought to develop and create a cognitive niche comprised of properties to produce religious beliefs and activities - and consequently these beliefs and activities have shaped the religious niche. Indeed individuals and societies actively engage in the maintenance and construction of religious worldviews via the use of material culture. 


\section{Chapter 2: Evolutionary Psychology: An Overview}

\subsubsection{Introduction}

Supporters of the Standard Model view religion as a product of cultural transmission. Yet characteristics unique to our cognitive machinery serve a regulatory function, particularly with how these cultural representations are processed in the brain. Religious behaviour is seen as a perennial feature of human behaviour. According to the Standard Model, belief systems look similar crossculturally due to universal features within our cognitive architecture. The contents of religious representations are similar in their expression because we uniformly process information about the world via certain innately bound cognitive sub-systems. For example, the prevalence of 'anthropomorphic beings,' within religious belief systems, may be due to certain innate cognitive features of the human mind. Subsequently, identifying the evolved neural correlates which determine the characteristics associated with religious behaviour remains important to this Standard Model.

This chapter will describe a model of mind that has influenced the theories and methods employed by the Standard Model. Psychological nativism looms large in the literature associated with the Standard Model and it is Evolutionary Psychology (EP) which has 
had the most profound influence. Cultural representations are transmitted between brains. Consequently brains regulate the processing of cultural representations in particular ways. EP argues that because our neuro-physiology is the result of evolutionary processes, then the cognitive constraints which regulate the processing of information are due to inferences surrounding natural selection. As a simple example, our general fear of spider's maybe an adaptive trait inherited from our Pleistocene ancestors living in the wild. Further, behavioural trends such as xenophobia maybe due to innate brain function. Beginning with a general overview of cognitive anthropological approaches to cultural transmission, the following will outline what has now become the more orthodox model of EP.

\subsubsection{Cognitive Anthropology}

Dan Sperber (1985) notes that the division between anthropology and the cognitive sciences is commonly founded upon by a disagreement over the facts regarding explanations for and interpretations of cultural phenomenon. Within anthropology the causally relevant features of culture are defined by the collective distribution of external/symbolic properties. Whereas within psychology the causally relevant features for culture available for explanation remain as purely psychological facts. Within anthropology it is assumed that the content and organization of cultural representations should be viewed as an "autonomous level of reality" 
existing as features outside the cognitive processes of the individual mind (Sperber 1985:76). Thus traditional anthropology views any attempt to develop an explanatory psychological model for the causal efficacy of cultural properties as mistaken. On the other hand, psychological explanations have typically argued that it is purely mental properties that make the expression of culture possible. Thus on this view psychological facts are more relevant to an understanding of human cognition than are cultural facts.

However Sperber sees the distinction between the external and internal processes that constitute cultural representations as misguided. Both brains and environment cannot be assumed as ontologically distinct categories of study. For Sperber, anthropology fails to address minds - whereas psychology fails to address environments. Indeed on Sperber's view: "Cultural phenomena are ecological patterns of psychological phenomena" (Sperber 1985:76). The question for researchers should then be: why is it that we see certain cultural representations as being widely distributed within a population more so than others? Sperber believes an explanation can be found by merging the two disciplines: psychology and anthropology.

The spread of certain cultural representations can be explained on Sperber's view as being caused by an interaction between minds and environment. Sperber argues then that our cognitive architecture is 
likely to be vulnerable to the transmission of certain cultural properties over others. Because psychological facts will inevitably determine the frequency and spread of representations Sperber offers an 'epidemiological' model of culture. Analogous to the spread of viruses, the successful spread representations will inevitably depend upon the mode of transmission i.e. an organism's susceptibility to the contagion and its transmissive frequency within a population will inevitably determine its virulence.

However, Sperber distinguishes between two forms of cognitive processing: mental representations that are intra-subjective and internal to the information processing device; and public representations - which are inter-subjective and external to the device. Private (mental) representations are hence dependent upon only thought and memory. Whereas public representations require individuals to actively modify their environment in order for them to become shared. Once representations are public they can be inferred by others and made available for abstraction.

However there is no guarantee that the cognitive processes which enable the transmission of cultural representations from agent-toagent will ever be accurate. A mental representation of publicly represented data will more than likely be subject to the cognitive "filters" which normally regulate psychological inferences. Thus the successful spread of a public representation will depend upon the 
capacity to induce a particular type of mental representation that is accurate enough in order for it to be transmittable. Indeed on Sperber's view inferential processes inevitably shape the cultural topology of a collective. Consequently the transmissive frequency of a cultural representation remains dependent upon information being able to be processed successfully and passed on. This thesis supports the core (and mainly correct) features of this model, particularly the notion that minds do indeed regulate the processing of information.

\subsection{Evolutionary Psychology}

On Sperber's view, the stability of culture and the universal features underlying cultural features can be explained primarily by an appeal to our cognitive architecture (Sperber and Hirschfeld 2004). As an anthropologist, Sperber is obliged to recognise the range and variation of cultural traditions as reflected in the ethnographic literature, while as a cognitive scientist Sperber pays adequate recognition to the nativist position, which holds that certain behavioural traits are inbuilt as biological endowments. Arguably though - on the surface -there appears to be a great deal of diversity within societies and cultures, yet the constraints that cognition provides ensures that a considerable number of cultural categories remain comparable in their expression. Thus Sperber acknowledges the importance that our evolved cognition plays in the transmission of 
cultural traits. Indeed Sperber remains the first of many evolutionary psychologists whose examination of culture has been notably shaped by the Fodorian modularity thesis ${ }^{2}$ (Fodor 1983).

Within Evolutionary Psychology [EP] however the brain is viewed as being comprised of domain-specific psychological mechanisms that have evolved to solve adaptive problems faced by ancestral lineage. Hence, cultural representations will inevitably be constrained to some degree by innate, adapted features of mind. This framework for EP however is founded upon the observation that a great deal of our "learning" is non-empirical (i.e. not learnt via first-hand exposure to the appropriate developmental resources). This is mainly an explanatory problem, as EP has observed that a significant number of our behavioural traits are underdetermined by social and cultural learning. Hence there is a 'poverty of the stimulus' in learning development which explains the existence of dedicated learning systems - dedicated learning systems which have genetically inherited.

The argument for the poverty of the stimulus emerged most prominently by way of Noam Chomsky's (1955 / 1975; 1988) models on linguistic nativism. Chomsky proposed that localized language abilities are functionally independent, comprised of what Chomsky

\footnotetext{
${ }^{2}$ Though it must be recognized first-and-foremost that the Fodorian thesis on Modularity has been quite influential to Evolutionary Psychology it is still quite different in its approach. EP's emphasize 1) the importance that evolution has on selecting for certain traits (psychological mechanisms), whereas Fodor has examined theoretical framework only sparsely. And 2) EP endorses domain specific psychological mechanisms that are modular, whereas Fodor argues for a central processor that integrates these epistemically bound perceptual systems which are much more encapsulated.
} 
calls a language-specific "mental organ". His argument rests on the notion that a child's cognitive development in regards to languagelearning is supported by an innate universal grammar (or the product of a generative grammar). In regards to language, children command an infinite generative faculty that emerges from informationally impoverished environments incapable of bestowing its rich and subtle intricacy.

Subsequently, researchers within EP suggest that this cognitive "division of labour" should apply on par to all other information processing systems in our cognitive architecture. Consequently, generative grammar which supports the notion of a dedicated faculty for language-learning has influenced a view of the mind as being comprised of other specialised systems which produce tacit behavioural traits. For EP Chomsky's picture of a language faculty should inform an explanatory framework for identifying further contentrich systems in the brain also. Indeed the orthodox models within EP view the mind as being comprised of functionally independent modules.

Committing to the view that natural selection has played a role in our evolved cognitive architecture supporters of EP are additionally committed to the position that these domain-specific processing systems or devices are innate and universal. Thus our brain like any organ in the body is viewed in Evolutionary Psychology as being 
shaped evolutionary processes (See Barkow, Cosmides and Tooby 1992; Pinker 1997 for overview). Domain-specific "modules" are viewed as innately specialized, content-rich psychological mechanisms that are adapted to process and manage specific classes of problem-solving. EP claims that this is why humans especially during early development - display a rich intuitive level of competence in certain behavioural domains over others.

Hence evolutionary psychologists' John Tooby and Leda Cosmides (1992) have proposed that the mind should be seen as a kind of computational juke-box with numerous songs in its repertoire (Tooby and Cosmides 1992:116). Subsequently, any song played at any given time is determined by the location, time and stimuli the jukebox encounters a particular cue in its environment. Agent's who are exposed to certain stimuli will respond in a certain way. This they propose illustrates that:

"[The] generation of this distinctive, culture-like pattern involves no social learning or transmission whatsoever. This pattern is brought about because like humans, the juke boxes (1) share a universal, highly organized architecture that (2) is designed to respond to inputs from the location situation" (Tooby and Cosmides 1992:116).

Hence Cosmides and Tooby argue that generation of "culture-like" patterns "involves no social learning or transmission whatsoever" (Tooby and Cosmides 1992:116). On this view then culture is evoked. 
Further, the argument for domain-specific psychological mechanisms versus a "general purpose" problem-solving system is that human agents tend to be very adept at a restricted class of tasks. A brain with domain specific algorithms would have the luxury of their information processing systems being routinely activated when in range of a specific class of problem. Cosmides and Tooby (1987; 1994) suggest that a domain general system would be too computationally sluggish in any given task domain. A domain-general system would be burdened with having to evaluate all alternatives potentially available to a problem solving class. Indeed a domain general lacks "content", or the in-built knowledge (i.e. algorithms) required to guide an organism within a specific task-solving domain.

\subsubsection{Folk Cognitive Systems}

Thus EP suggests that the reliability and relevance of the information being available to an agent's prediction-and-control system depends on the information-processing system's level of specialization. According to EP the algorithmic processors underpinning certain cognitive traits are going to be more robust than others. For instance our naïve or folk understanding of natural world dynamics EP argues can be attributed to a content-rich system that intuitively registers the world and guides perception. Outlined below are some of the traits associated with this expertise: 
Naïve/Folk Physics: Cognitive psychology has observed that even at a very young age, children understand intuitively the physical dynamics of continuity and solidity (Spelke, 1990; 1998). They also require a minimal amount of learning to understand the physical relationship between gravity and weighted objects (Schilling \& Clifton 1998) and infants can manipulate physical objects quite adeptly in goal oriented tasks (Povinelli 2000) [See above for discussion]. The 'native' algorithm for interpreting the physical world generated by our evolved cognitive architecture puts human agents at an advantage over other non-human primates whose 'physical assumptions [about their world] are grounded in [their] perceptual generalizations' (Boyer 2005:109). Daniel Povinelli (2000) and colleagues have demonstrated the clear advantage that human infants have over chimpanzees in solving tool-use problems. Their experiments show that human infants were not only capable of intuitively acting on their conception of the physical world successfully, but they were also much more flexible when the problems faced were modified.

Naïve/Folk Biology: Children at an early age can recognize the ontological difference between biological and non-biological objects and can rationalize in distinctly different ways about them (Keil 1986). Moreover, Scott Atran (1998) has suggested that we possess the capacity at an early age to distinguish various taxonomic categories: "Humans everywhere think about plants and animals in highly structured ways. People have similar folk biological taxonomies composed of essence-based, species-like groups and the ranking of species into lower- and higher-order groups. Such taxonomies are not as arbitrary in structure and content, nor as variable across cultures... These structures are routine products of our 'habits of mind,' which may in part be naturally selected to grasp relevant and recurrent "habits of the world"' (Atran 1998:547). Thus there is a 
folk sense attribute to living and non-living things. Our intuitive understanding of biological categories is "essence" driven to the extent that humans can intuitively distinguish between natural categories - an intuitive taxonomic categorization system. Humans by default organize living things into identifiable order and ranking groups i.e. plants and animals are living things, but they are naturally categorized as different groups of living things.

Naïve/Folk Psychology: False-belief tests have demonstrated that children after the age of four reliably understand others as possessing belief and desires (Leslie 1987; Leslie and Frith 1987). False belief tests usually assess the ability of children to distinguish the epistemic differences between their own beliefs and the beliefs of others' ${ }^{\prime 3}$. Children natural attribute immaterial substances, beliefs and desires, to the minds of those around them. They understand that others may be mistaken about the world, and develop a robust distinction between private and public knowledge. For example, as false-belief tasks have illustrated seemingly universal constraints in the development of our interpretive capacities (Scholl and Leslie 1999; Baron-Cohen, et al 2000; Perner, Leekam, \& Wimmer 1987). Moreover Deborah Keleman has shown that young children understand the world in a teleological mode constituted by intentionality (Keleman 2004). Indeed the developmental data points clearly in the direction of rich innately structured knowledge of other minds. (BaronCohen, et al 1997). Put simply, mature humans deploy tacit assumptions about the inner- cognitive states of others in real-

\footnotetext{
${ }^{3}$ Versions of false belief tests differ, but the most common one consists of a child observer watching someone (let's call her Mary), putting a doll inside of a box. When Mary leaves the room, however, someone enters and moves the doll from the box to the cupboard. When Mary re-enters the room the child observer is asked where they think Mary thinks the doll is. Children under the age of 4 usually answer that Mary thinks the doll is where it was moved to last - the cupboard. More mature children between the ages of 4-5 recognise that Mary thinks the doll is still in the box. They know that Mary has a 'false-belief' regarding the whereabouts of the doll.
} 
time and accurately because we possess the information processors necessary to do so (Pinker 1997: 329-333).

\subsection{The Disembodied Computer}

The EP paradigm has significantly shaped the cognitive science of religion. Religiosity is currently studied via the evolutionary sciences as a uniquely human cognitive trait, especially since it appears to be ubiquitous to all cultures. Yet before providing a broad overview of the cognitive science of religion it would be prudent to sum-up some of the principles associated with EP: Firstly, EP sets out to identify the unique selection pressures which have caused the unique cognitive traits that Homo Sapiens possess. EP highlights those particular cognitive traits that supported the inclusive fitness of the hominine ancestral lineage, thus evolutionary processes should be viewed as fundamental to our understanding of human nature and culture.

Secondly, thought processes and culture is evoked. In other words, cultural representations are a product of the information processor that has evolved to respond to a variety of environmental cues. For instance, according the Standard Model cultural artefacts don't extend the capacity for religious thought. Instead they are used to trigger certain innate mental inferences: "More generally, religious concepts too constitute salient cognitive artefacts, whose successful cultural transmission depends on the way they activate our inference systems in particular ways" (Boyer 2002:153-154). Lastly, the cognitive 
modules which have evolved to respond to these cues are adapted to specific task-solving domains. Consequently, certain behavioural traits are reducible and considered universal cross-culturally.

It is important to recognise then that because EP views the brain as being suited to a particular environment of evolutionary adaptedness [the EEA] that the information processor that has evolved remains preset to this statistically probable time and location. Thus, despite the human cognitive architecture's operation in a variety of contexts, this 'disembodied computer' remains a priori to the agent's current socio-ecology. This paradigm is sometimes view as a representationalist theory of mind, as it tries to explain "how disembodied 'internal' ideas can represent 'external' physical objects and events" (Johnson and Rohrer 2006:17). In other words, its explanatory target is driven by an understanding of how brains (the inner world) regulate representations of the external world.

Though this is not necessarily a mistaken view of human cognition it does however tend to advocate a Cartesian understanding of brain and body. Indeed the brain-as-computer remains disembodied within its cultural setting. These dualist models often view the properties associated with cognition as ontologically distinct from the environment where the agent is situated (see Johnson and Rohrer 2006 for overview of debate). Thus the brain-as-computer is viewed as operating within a set of "universal logical rules that govern the 
manipulation of 'internal' mental symbols, symbols that are supposedly capable of representing states of affairs in the 'external' world" (Johnson and Rohrer 2007:19). As a result it is often assumed that the cognitive processes which govern "manipulation of internal symbols" can be regarded as detached from the environment/context where cognition emerges.

The computational metaphor has underpinned EP models. It is proposed then by EP that the cognitive system that has been shaped by the EEA functions under a set of logical rules which are genetically embedded. Thus the behavioural output of agents is adapted to a specific time and place in our evolutionary history. The predetermined nature of our cognitive capabilities are seen by supporters of EP then as detached from the current set of circumstances that human beings find themselves in. This is only partly true and what follows will be an evaluation of this premise. I will argue in chapters 4 and 5 that the scope and nature of cognitive development is not only functionally plastic, but dependent on the recruitment of non-genetic resources (i.e. cultural artefacts) for mental reasoning and thought. Indeed culture is not just epiphenomena - nor is it evoked. Instead the neurological resources - that are genetically inherited - are additionally supported by culturally inherited resources.

But before proceeding with this evaluation of EP (in Chapter 4), I will first introduce the reader (in Chapter 3 ) to what is regarded as the 
Standard Model within the cognitive science of religion (see Boyer 2005; see also Day 2005; Whitehouse 2005). So far I have focused on some of the key concepts upon which the Standard Model is founded. For instance, the theoretical concepts highlighted by the authors in the next chapter converge with Cognitive Anthropology and Evolutionary Psychology primarily in two ways: Firstly, cultural representations are transmitted between agents. Nevertheless these representations are subject to the regularities of our native cognitive processes. Hence, a causal explanation for the recognition of persistent cultural patterns will involve identifying features of our cognitive hardware that determines cultural selection.

Secondly, humans are equipped with unique cognitive systems which allow us to track the world in a particular way. These specialised "folk" systems have evolved as adaptations and constitute our perception of the world, yet they also influence and determine the transmissive frequency of cultural representations. Standard Model supporters argue that while religions across the world may appear to be differentiated, they are nevertheless comprised of common, underlying features. These universal patterns of culture can be explained, it is argued, with an appeal to innate cognitive systems. 


\section{Chapter 3: The Standard Model}

\subsection{Implicit versus Explicit Religious Representations}

The Standard Model (SM) of religious cognition is partly motivated by an explanatory concern. The phenomenological facts that constitute religious representations cannot necessarily inform how those representations are processed in the minds of individuals. Thus explicit representations (i.e. the kind of "god" one believes in) are not so much the focus of explanation as the cognitive machinery that generates them. Though a religious agent maybe able to rationalize their belief or experiences in a certain way, it is assumed that this rationalization is the result of certain cognitive rules governing the expression of religious representations. Implicit or intuitive constraints regulate explicit forms of religious reasoning.

For instance, in experiments conducted by Justin Barrett and Frank C. Keil (1996) participants were read a narrative whereby a deity responded to the prayers of a person in need. They were also asked if this particular deity was omniscient and omnipresent. Those who answered 'yes' to this question also recounted the story in a way that contradicted their conception of an omniscient being. An example of one of the narratives (in short form) went like this: God is playing scrabble with an angel. A woman in Brazil is in trouble and she prays to god for help. God stops playing scrabble with the angel and 
answers the woman's prayer, then returns to the game of scrabble. In explicit reports of god's actions during the narrative it was made clear to Barrett and Keil that individuals - despite believing in god's omniscience - tended to recall that god made two actions. He stopped playing scrabble then answered the woman's prayers. Consequently Barrett and Keil found that religious representations are processed by employing the normal cognitive resources associated with everyday (or folk) inferences online. Though god maybe viewed as having supernatural qualities, the participants nevertheless processed the narrative version of god as having natural properties: the 'god' character conformed to a spatial and temporal location.

Studies such as these have been used to illustrate that despite a believer's conceptual understanding of a supernatural being (say their omniscience), there nevertheless exists an inferential constraint on how agents relate cognitively with their perceived belief in a god or gods. Hence agents still tend to conceive of omniscient gods with anthropomorphic properties for instance (i.e. as though they are human agents) (Barrett and Keil 1996). Barrett argues then that theological concepts - such as a god's omniscience, or afterlife concepts - are often too computationally difficult to manage online, despite our capacity to communicate an explicit understanding of a god's properties in a symbolic sense (see Barrett 1999). 
This perspective suggests that religious reasoning operates at two different levels of cognitive processing: implicit and explicit. Though individuals express their beliefs and practices in a variety of ways, underlying this behaviour are specific psychological regularities. Explicit forms appear to be ruled by implicit constraints. The Barrett and Keil experiments show that religious reasoning is constrained by default inferences. Though a belief in an omniscient god may be due to the relevant exposure to during development, religious agents nevertheless intuitively recall the properties of their god or gods as conforming to natural rules. Essential to the SM view is that there exist innate cognitive systems which regulate religious representations. Subsequently, underlying religions cross-culturally are universal characteristics which are constant.

The SM model not only agrees that there are recognisable consistencies within religions, but the SM has also set out to offer a causal explanation for these patterns. For instance, why are the majority of gods, deities, spirits, demons etc anthropomorphic in their appearance? Why do they often possess 'super'-natural powers? Why do supernatural beliefs persist despite the secularization of modern societies? And fundamentally: are humans predisposed to engage in religious beliefs and practices? The SM is motivated particularly by the last question. Researchers within the cognitive science of religion have often defined the focus of their research by arguing that the reason why we see commonalities across religions is 
because all humans share something in common: biologically endowed features of our cognitive architecture.

3.2.1 Pascal Boyer: religion as a cognitive by-product

One of the leading figures within the SM is Pascal Boyer (1994, $2001)^{4}$. Boyer takes seriously the claim made by EP that human cognitive architecture is comprised of functionally independent systems traditionally called "intuitive" or "folk" domain-specific processors (see above). The cognitively impoverished environment that infants are raised in suggests that the inference engines that guide behavioural output must be to varying degrees innately driven. Subsequently Boyer argues that the learning and absorption of information is regulated via inferential processes governed primarily by our cognitive machinery. In addition, Boyer notably suggests that a universal feature of religious belief systems is that they are comprised of representations that are salient and thus memorable to our native cognitive architecture.

Similarly to his teacher Dan Sperber, Boyer argues that certain representations will be more prevalent than others due to inborn perceptual biases that are regulated by our evolved cognitive architecture. Hence on Boyer's view: "to explain religion is to explain a particular type of epidemic" (Boyer 2001:53). Indeed Boyer asserts

\footnotetext{
${ }^{4}$ For a comprehensive yet straightforward introduction to Boyer's theoretical approach see Religion Explained: The Human Instincts That Fashion Gods, Spirits and Ancestors [2001]) which establishes itself within a cognitive anthropological model and EP [see above]).
} 
that human cognitive architecture is highly susceptible to religious ideas and beliefs and establishes his epidemiological model of religious cognition with an appeal to EP's examination of our innate folk perceptual biases (i.e. folk psychology, folk biology, folk physics [see above]). Our cognitive architecture is comprised of domain specific algorithms that guide and direct our interpretations of the world.

For Boyer, then, supernatural concepts appear relevant and exciting to our cognitive architecture precisely because religious ideas normally run "counter" to any real-world data that our folk systems have evolved to track. Indeed Boyer assumes that our natural inference engines constitute a robust foundation for the epidemiology of religious representations. It is because religious ideas stimulate a number of innate ontological categories that they possess a selective advantage over other competing cultural representations. Accordingly, because religious representations are "counterintuitive" to our common-sense perception of the world their cross-cultural success is explained by our mind's propensity to be aroused by these "exciting" concepts.

\subsubsection{Minimally Counterintuitive Concepts}

Importantly for Boyer and others who endorse the 'Standard Model' [SM] there is no natural place for religion in the brain, nor is 
there any dedicated cognitive system for religious behaviour. Instead religious representations are normally viewed as a by-product of other evolved cognitive features (i.e. religion is a mental spandrel ${ }^{5}$ ). Put simply, because religiosity does not serve any adaptive function it is viewed as derivative of other evolved features. For example, my nose is not adapted to holding the glasses I wear upon my face, yet it serves this purpose anyway. For religion however, it is argued, that certain cultural representations are more exciting to our native cognition because they break with certain ontological conventions making them more appealing. Thus it is precisely the counterintuitive features that make religion salient and memorable to us. Indeed Boyer argues that supernatural representations are counterintuitive because they are comprised of conceptual properties that are:

Counterintuitive to folk psychological inferences: The gods are normally interested in what we do and think, thus on Boyer's view the gods are often represented as "Full Access Strategic Agents" (Boyer 2001). Indeed a supernatural entity's "strategic access" runs counter to our understanding of mentality, as our normal intuition or understanding of minds is that they are private and inaccessible.

Counterintuitive to folk biological inferences: Conceptually, supernatural representations run counter to our natural inferences regarding biological categories e.g. the gods are immortal, and can embody sacred animals or people.

${ }^{5}$ See S. J. Gould \& R. Lewontin, "The Spandrels of San Marco and the Panglossian Paradigm: A Critique of the Adaptationist Programme," Proc. Roy. Soc. London B 205 (1979) pp. 581-598 
Counterintuitive to folk physical inferences: Supernatural concepts run "counter" to our natural inferences regarding the physical dynamics of the world e.g. the gods inhabit the sky, or are omniscient.

However, while Boyer recognizes that supernatural concepts are counterintuitive to our inferential machinery they are nevertheless only "minimally" counterintuitive. They violate one or a few tacit assumptions, but not many. This feature enables religious concepts to be cognitively tractable, salient, and memorable. Pile on the violations, and religious concepts will cause a mental gridlock. For Boyer supernatural representations conform to a minimally counterintuitive $[\mathrm{MCl}]$ basin of attraction in two ways:

Firstly, explicit MCI concepts are comprised of minor breaches on intuitive knowledge. Thus ancestor spirits are both people as they possess intentionality (i.e. beliefs and desires) yet they do not conform physically to our intuitive expectation of what people do: the ancestors are invisible and can inhabit sacred places and objects. In addition, the ancestors are alive while being dead, thus violating our intuition regarding properties associated with our folk biological inferences.

Secondly, $\mathrm{MCl}$ concepts are constituted by a transfer between folk domains. Ontologically, the gods can be both people and inanimate objects at once - both a psychological property and a physical property.

In addition physical items (such as sacred alters or totems) can embody counterintuitive properties also. Yet religious artefacts 
additionally conform to the physical constraints that an inanimate

object is naturally subject to. However, a sacred object can appear

intentional in respects to our folk psychological inferences as well.

For example, healing rocks may appear to have an intentional quality

to them - their purpose is to cure sickness. Indeed healing rocks may

conform to an intuitive notion of agency, as they are often perceived

as humanly significant to the lives religious actors ${ }^{6}$.

For Boyer then the epidemiology (or dominant spread) of certain representations are contingent on two properties constituting an $\mathrm{MCl}$ concept. $\mathrm{MCl}$ concepts are a violation of expectations (i.e. the transgression of normal ontological categories), plus $\mathrm{MCl}$ concepts are also constituted by some non-violated assumptions that are tacitly activated (i.e. supernatural concepts remain grounded still in a relevant ontological category). In short, religion is the familiar made strange - but not too strange.

Pascal Boyer and Charles Ramble (2001) have tested this hypothesis on subjects who were asked to read narratives containing

${ }^{6}$ This cross-networking of intuitive domains is currently being supported by research into the learning development of children. Deborah Kelemen (2004) for instance has pointed to evidence that suggests that children from a very early age have a tendency perceive non-biological objects as purposeful and endow them with intentionality. Keleman has demonstrated that children during early development have a "promiscuous teleological" tendency, in that they possess a preference for intentional explanations regarding natural phenomena over-and-above explanations that refer to a autonomous evolutionary process (i.e. natural selection). Keleman observed that children are more likely to define natural phenomena as having a purpose, such as pointy rocks are purposefully designed for animals to scratch there backs with (Kelemen, 1999c; but see Keil, 1992). Further, children possess a preference for explanations that entail a sort of "creationist" or designer bent. She cites studies performed by E. M. Evans on American children both religious and non-religious who mostly preferred creationist explanations for species over and above evolutionary stories (Evans 2000). She suggests that these intuitive tendencies make it more likely that children will be more susceptible to religious concepts from their own culture and hence establish intentional inferences in relation to animate and inanimate properties. 
counterintuitive at differing levels of complexity (i.e. extremely minimal in their content to maximally counterintuitive concepts). In the experiments conducted on the memorable features of certain narratives it was the $\mathrm{MCl}$ concepts that individuals could recollect better over the other counterintuitive concepts. Predominantly when readers were expected to recount the narrative of certain stories, the $\mathrm{MCl}$ concepts were better recalled by participants. From this both Boyer and Ramble (2001) have constructed a model that aims to predict the relative success of religious concepts. Optimal concepts contain:

[1] A pointer to a particular domain or concept

[2] An explicit representation of a violation of intuitive expectations either:

[2a] A breach of relevant expectations for the category, or [2b] A transfer of expectations associated with another category;

[3] A link to (non-violated) default expectations for the category

Hence: [1] can be a pointer category to an agent or artefact. For instance, agents such as ghosts typically run counter to our commonsense assumptions regarding physical properties (they can walk through walls and solid objects). This constitutes a breach of relevant expectations [2a]. Whereas an artefact may exploit our intuitive expectations of embodiment: hence a statue of the Virgin Mary who can hear others' prayers constitutes a transfer of psychological 
agency mapped onto a physical property (i.e. the cross-networking of two distinct ontological categories). Hence artefacts that conform to this mould would typically match [2b].

While there is a counterintuitive element to both these examples, there are properties that still remain grounded within an ontological category. Hence [3]: a ghost conforms, or is grounded in, our folk psychological understanding of other people. However, while an artefact conforms to our understanding of both: a) ordinary physical properties and b) psychological properties, its counterintuitive status is defined by the cross-transference of ontological categories. Thus the "gods" for Boyer appear to conform to an intuitive concept of some ontological category (providing a conceptual comprehension) while transgressing common-sense inferences of the world in other domains (providing it with a mnemonic advantage).

\subsection{The Naturalness of Religious Cognition}

Because our cognitive architecture is seemingly more susceptible to $\mathrm{MCl}$ concepts it is assumed within the SM that religious cognition is consequently a rather natural cognitive bias. Those within the cognitive science of religion who endorse this position tend to reduce this "naturalness thesis" down to a learnability factor (See Barrett 2000; McCauley 2000). Similar to the nativist conception of linguistic competence (or generative grammar), which argues that language 
skills are an inbuilt competence, religiosity is seen as being a natural feature of human development and emerges somewhat independently of learning. However the development of religious behaviour occurs despite the fact that - unlike language abilities religiousness is not viewed by supporters of the SM as being the product of an inbuilt and specialised cognitive system, but as a byproduct of other evolved features in the brain.

Boyer's argues that we should expect counterintuitive concepts to conform to these rules cross-culturally. The basic properties that motivate our intuitive inferences and ensure the efficacy of a god in one cultural setting will in all likelihood make them interesting in another. Thus we should view the transmission religious representations within the SM as being necessitated by a 'cognitive optimum'. On this view, religious representations should have a selective advantage due to their counterintuitive qualities. However supernatural concepts that gain a greater transmissive frequency will also be less computationally intensive. Thus the supernatural concepts prevalent in religions are both "interesting" while being cognitively easy:

"In this framework, a religious idea would be described as cognitive \& optimal if (i) it contains an explicit violation of commonsense thinking and (ii) it makes implicit use of the intuitive principles of commonsense knowledge" (Boyer 1992:45). 
In short, our evolved brain constrains the appearance of cultural traits. Religious belief systems are subject to the cognitive processes that regulate their expression. Again, religions are cross-culturally similar according to this view because our minds remain preset and attracted to certain concepts over others. So despite the required cultural inputs, religious representations are governed by innate cognitive rules that determine their expression. Hence supporters of the SM suggest that religious belief systems - despite their variability - can be reduced to a certain number of properties (see Boyer above). Yet the "naturalness-of-religion" hypothesis proposes that the basic profile of human cognition makes "religion" rather intuitive and easy (Barrett 2000). Like language then human cognitive hardware seems well suited to generating religious ideas. As Justin Barrett (2000) has pointed out:

"Much as language is naturally acquired as a result of cognitive preparedness plus exposure to a typical sociolinguistic environment, ordinary cognition plus exposure to an ordinary environment goes a long way towards explaining religion" (Barrett 2000:29 [emphasis added]).

One may come to the conclusion then that religion is constituted by a 'natural' disposition to view the world religiously (in some respects). Religious information is not only easier to process and comprehend our perceptual bias for religion indicates that it would be difficult for individuals to anything but religious according to this naturalness 
thesis. Thus we are not only predisposed to religious "ideas", the very classification of religion is defined by our innate predispositions and the manner in which it tracks the world.

\subsubsection{McCauley's Comparison}

Similarly, Robert McCauley (2000) makes a comparison between both "natural" and "unnatural" properties of culture by illustrating the differing ontological and epistemic qualities of religion and science. He suggests that we are far better suited for processing religious information than scientific information. Science is difficult to process, requires substantial training and discipline, and contrasts in many ways to our intuitive picture of the world. On the other side, religion is virtually inevitable - a kind of mental plaque. In light of this comparison McCauley raises two methodological questions: Firstly, to what extent are cultural arrangements the cause of the behavioural patterns typical to a particular characteristic; and secondly to what extent we can explain cognitive traits via "normal" cognitive processes independent of cultural factors:

"Some cognitive capacities seem to turn neither on any particular cultural input nor, as in the case of face recognition, on any peculiarly cultural input at all. Children's proclivity to acquire language and nearly all human beings' appreciation of some of the basic physics of solid objects, their assumptions about the mutual exclusivity of taxonomic classes in biology, and their abilities to detect 
and read agents' minds are just some of the proposed candidates for human cognitive capacities that arise independently of any particular cultural input" (McCauley 2000:63).

Whether religion is natural or unnatural for McCauley will depend upon its mode of expression - relative to certain cultural inputs. Indeed McCauley argues that since religion is a historically pervasive phenomenon, whereas as scientific institutions are not, then religion probably requires precious little social, cultural and technological support for its presence:

"In calling religion "natural" and science "unnatural" in this second sense, I am suggesting two things. First, the elaborate cultural institutions surrounding each play a far more integral role in the generation and persistence of science than they do in the case of religion... Second, most of the cognitive activity underlying religion concerns cognitive processes that rely far less on particular cultural input, particular forms of cultural input, or even peculiarly cultural input than is the case with science" (McCauley 2000:64).

Elaborating this claim further, McCauley argues that the institution of science remains potentially fragile to our natural cognitive biases, which are normally inclined to view the world according to predefined frames. Thus, according to McCauley's comparison, science requires a greater level of socio-cultural learning and support in order for it to be sustained. Within scientific enterprises the requirement is that: "(1) 
scientists develop explanatory theories that challenge received views about empirical matters and, (2) their critical assessment of those theories highly values evidence born of empirical tests" (McCauley 2000:69). Yet in order to develop explanatory models not underdetermined by the evidence, scientists must lean heavily on concepts that often run counter-to their normal perceptual biases.

Non-intuitive concepts like scientific ones are always vulnerable to corruption from ideas that are easier to learn and comprehend such as religious explanations for natural phenomena. In order to override the competitive advantage that religious concepts have over scientific ones, McCauley suggests that a greater concentration of institutional presence is required. Hence scientific institutions are unique to a particular historical context precisely because of the selective advantage that religious representations have over scientific concepts. In contrast to science:

"Religion dates from our prehistoric past. Both the archaeological record and the anthropological evidence shows that human religious activities do not depend on keeping chronicles or on inventing writing or even on establishing fixed settlements...Thus neither the birth nor persistence of religion critically depends on any special cultural conditions" (McCauley 2000:74).

Beyond its difficulty, McCauley notes that science searches "for accounts of reality that are more comprehensive and discerning for 
which the production of evidence requires progressively more rarefied circumstances" (McCauley 2000:71). Again, this claim is made in contrast to religion which is less theoretically demanding and less critical of its explanatory concepts: "Religious truths are primarily to be retained and transmitted rather than reflected on and challenged. The crucial point is that neither comprehension nor retention of religious materials requires development or possession of any sort of specialized intellectual skills on which both the acquisition and the progress of science depend" (McCauley 2000:76). Finally, McCauley urges that science is more intellectually demanding of its adherents than religion is:

"The acquisition of scientific knowledge is a painstaking and laborious process... Not only is scientific knowledge not something that humans acquire naturally, its mastery does not guarantee that someone will know how to do science. After four centuries of astonishing accomplishment, science remains an overwhelmingly unfamiliar activity, even to most of the learned public and even in those cultures where its influence is substantial" (McCauley 2000:71).

For McCauley religion is intellectually much easier in comparison to the intensive knowledge-base requirements for scientific enquiry: "Acquiring the knowledge necessary to participate in a religious system is much more like acquiring a natural language than it is like mastering the knowledge and skills necessary to do serious science. Acquiring religious knowledge requires little, if any, explicit 
instruction" (McCauley 2000:80). Thus McCauley echoes Barrett's earlier statement when he says:

"Like natural language religion exploits cognitive dispositions, which seem to arise early in human development. Because so many pivotal religious conceptions have so little theoretical depth, possessing everyday concepts prepares people for the acquisition of religion in a way that does not prepare them for the acquisition of science" (McCauley 2000:80).

On McCauley's view religiosity as a behavioural domain consists of a structured system relative to individuals' commitment towards culturally postulated superhuman (CPS) agents (similarly Boyer calls supernatural beings counterintuitive concepts of agency). Representing and acquiring CPS agents, is a relatively basic ability once one recognizes the innate cognitive mechanisms that produce supernatural concepts. Firstly, by the time we have reached a stage in early childhood we have become seasoned 'anthropomorphizers' (See Guthrie 1993). Even at an early age our default position is to perceive human agency in non-human and inanimate events and artefacts. This natural compulsion to project agency appears to influence our tendency to perceive the gods as person-like despite the theological position to the contrary.

Secondly, our overactive capacity to "mind-read" within a social context (i.e. make inferences about the intentional states of other 
agents) ensures that the attribution of psychology agency - where there is none - remains more likely. Subsequently, humans will tend to make inferences about our natural world as being humanly significant (e.g. a famine is god's punishment for our sins). Thirdly, narratives relative to religious systems are usually imbued with the ontological status of their CPS agents, making them easier to remember and transmit. Narratives concerning the beliefs, desires, thoughts and actions of CPS agents have a "mnemonic advantage" because of their content and structure. But they also provide individuals with the chance to make the theological properties of a CPS agent more relatable to agents. Myths and narratives thus attribute a god or gods' ontological status with a life history, substance and an explanation for their existence and purpose.

Finally, on McCauley's view, religion provides individuals cheaper information, or more particularly, explanations about the world which adhere to our cognitive biases. Religious systems render to individuals seemingly coherent information about the natural world that would normally be inaccessible without extensive cultural support (such as in the case of scientific explanations). It is easier to ascribe intentionality to, say, seasonal change (i.e. the god of winter) than it is to understand that the weather patterns exist independently of our tendency for teleological modes of thought (See Keleman 2004). Thus in contrast to science McCauley asserts that religious information doesn't really challenge our natural intuitions about the 
world. It maps very easily on to our natural perceptual systems and subsequently our default ontological understanding of our world.

McCauley argues that religion is far more natural than other "unnatural" cultural knowledge domains like science. Science, McCauley argues, is more "counter-perceptive" than religion is due to the abstract nature of its concepts. However our competence with religious information requires precious little cultural knowledge, as we appear to be well suited to managing a 'supernatural' dialectic with the world. Yet McCauley's premise adheres to the more pervasive assumption within the SM: that we do not necessarily possess a functionally independent system for processing religious information (i.e. a dedicated religion 'module' if you will).

\subsection{Summary and Conclusion}

I started off this chapter with an explanation for the difference between implicit and explicit religious representations - that there is a distinction between how religious concepts are explicitly represented and how they are represented in the minds of individuals. A lot of religious concepts, it is argued, are too computationally difficult to process (i.e. in the case of god's omniscience). Barrett and Keil's experiments show that the qualities of a particular god or gods, are not always represented in the mind as being supernatural (Barrett and Keil 1996; Barrett 1998). On the contrary, we often represent 
gods as ordinary people within a fixed temporal and/or geographical location. The supernatural qualities of a god seem to be relatively easy to recall when individuals discuss them or rationalize their beliefs, but these supernatural are lost on the subjects they looked at when they were forced to recount them in a narrative.

The SM approaches the psychological processes associated with religiosity by its examination of our evolved psychological architecture. Yet instead of viewing religion as a cognitive adaptation, supporters of the SM categorise religious behaviour as a by-product of other evolutionarily functional cognitive mechanisms. For the SM, religion is a pan-human trait precisely because we have evolved features in the brain which makes us more predisposed to certain cultural representations over others. Pascal Boyer argues that supernatural representations spread and are transmitted more prominently because they excite our native inferences regarding natural categories, because they are counterintuitive. We all come with the same cognitive hardware, it follows that we are all naturally susceptible to religious representations. For Boyer, religious ideas spread in an epidemiological sense because of the kinds of minds we possess. In Darwinian terms, religious representations have a selective advantage over other concepts, thus they are more likely to predominate within cultures due to this cognitive bias. 
However, the SM argues that while religious concepts are in some respects 'counterintuitive', they are only such to the extent that religious concepts meet particular conditions. Supernatural representations cannot be too counterintuitive (i.e. maximally counterintuitive as in the case of Robert McCauley's comparison with scientific concepts [McCauley 2000]), otherwise they would too cognitively difficult to process. Neither can they be under the threshold of what is deemed 'unnatural' otherwise they would not excite normal inferences. They must violate expectations, without causing a cognitive gridlock. A religious concept is constituted then by minimally counterintuitive properties. In other words, religious representations conform to a 'cognitive optimum'. As a result, religious beliefs are viewed by the Standard Model as intuitive and easy.

Robert McCauley holds a similar view: that religious information is cheap only because we appear to be well suited to providing explanations about our world in a 'religious' sense. In McCauley's comparison between religious and scientific concepts he showed that - historically - religious ideas have persisted and been more prevalent than scientific ones because we have a cognitive bias for religious worldviews. McCauley puts this down to the fact that science requires a considerable level of enculturation and cultural knowledge in order to be established, whereas religion does not. Both Boyer and McCauley then subscribe to a 'naturalness-of-religion' hypothesis: the 
view that the supernatural persists in cultures because our minds require little or no cultural learning to understand these ideas. Thus the epistemic load required to be religious is not all together burdensome. All that is required to be religious are the standard features of our cognitive hardware. 


\section{Chapter 4: Co-Evolutionary Models}

\subsection{Introduction: Cognitive Scaffolding}

The broader focus for researchers within the SM has been identifying pan-human regularities which govern the expression of religious belief. From what we know about the functional properties of the human brain, plus what we know about 'religion', researchers can form an explanation for why religion seems to be a cultural habit. In order to establish how human behavioural habits arise and persist, many have focused on the intersection between psychology and evolutionary biology. Evolutionary Psychology (EP), for instance, has chosen to focus on a probable time and location in hominin history during which these evolved traits became genetically entrenched (i.e. the Environment of Evolutionary Adaptedness, or EEA). Consequently, the properties of human cognition can be explained, according to EP by looking at the selection pressures which it subsequently adapted to. According to this view we possess a suite of specialised systems which have evolved to solve specific adaptive problems efficiently and effectively.

It will be argued that the domain-specific systems theory is overemphasized in EP. This chapter will present some alternative models of mind which challenge to the more orthodox perspectives within EP. Not only is the notion of genetically inherited psychological 
mechanisms an over-represented concept in cognitive nativism, it also under-represents some of the relevant cognitive processes and properties that are culturally inherited. Though it may be the received view that speech-language faculties are a standard biological feature amongst humans, it doesn't necessarily follow that the same is true for all of our cognitive and behavioural traits.

The received view within the SM is that religious behaviour - while not an adaptation - is a 'natural' disposition nonetheless. This chapter looks at other possible explanations for these cognitive processes and properties. I argue that the cognitive sciences have, for the most part, ignored research which has sought to explain the efficacy of agent-environment interactions. Instead of viewing agents as engaging with external artefacts as cognitive resources, the cognitive science of religion has traditionally sought to focus on representationalist computation. I argue that cognition emerges out of a particular interaction between internal cognitive resources and a modified environment. These modifications, in which agents adapt their environment to suit their own ends is sometimes called cognitive 'scaffolding' (Clark 1997; Sterelny 2003). The term scaffolding is employed within the cognitive sciences to describe the structured interaction between learning agents and the support for learning within their socio-cultural environment. We should view scaffolding as being a component in hybrid cognitive interactions. 
In the same way that a 'scaffold' is considered a supportive mechanism for construction purposes, human cultural environments are structured in a way to support the transmission of information for learning purposes. Indeed on this view agents inhabit a cultural niche comprised of "complex social structures that scaffold the individual by means of artefactual, linguistic and institutional devices" (Griffiths and Stotz 2000:45). For instance, coordinated interaction between agents and an external environment is the salient characteristic in developmental learning. Parents employ language, attentional activities, repetition and other structured teaching methods while educating their children. The same is true for most - if not all - forms of informational transmission.

Our learning environments are thus scaffolded in a sense that they are structured and include support mechanisms for development. For example, reading and arithmetic is a recent cultural invention in human history and is only available under certain environmental conditions. Reading and arithmetic occurs only when a learning environment enables the interaction between the naked brain and cultural practices. Humans are producers, reproducers and are subsequently produced by a scaffolded learning environment, in which the presence of external cognitive tools enables our day-today cognitive processes. The authors discussed below will contest cognitive nativism and endorse a cognitive 'constructivist' paradigm of 
sorts. It is argued that human cognition has co-evolved alongside the properties of culture which support hominine intelligence.

We do not depend upon internal cognitive resources alone. Instead our cognitive functions are enmeshed in a scaffolded learning environment whereby learning is facilitated by the artefacts of culture. We are indeed, as Andy Clark (2003) has stated, cyborg creatures that utilize the tools and devices of culture in order to support cognitive development. Part biological organism, part technology, the capacity to create tools that functionally support and augment our cognitive abilities is apparent in daily routines. My capacity to input encoded symbols into this document is not a genetically inherited trait, but a culturally inherited one. Typing is dependent upon me having the appropriate technological - and developmental learning resources are available.

\subsubsection{Dumb Brains, Smart Environments: Wideware cognition}

Andy Clark (1998; 2001a) has made a considerable impact within philosophy and the cognitive sciences with a representational theory of mind which he calls the 'extended mind' hypothesis. Clark recognises the vital role that external cognitive devices have had in the cumulative cultural evolution of human intelligence. Hence material culture is assumed as having as much parity to minds, as nervous systems have to brains. For Clark, the cognitive processor is 
not locked into the space between our ears, but actively external in the world beyond the cranium. Thus cognition within the extended mind model is seen to be a "continuous with processes in the environment" (Clark and Chalmers 1998:10). Material culture though deemed separate and measurable, is nevertheless actively incorporated with our cognitive expertise. Yet it is not our external world that is "smart" necessarily, but it is our tendency to lean on external media or external tools in order to get by. Thus Clark notes that while it is possible to numerically formulate simple mathematical problems, such as " $1+1=2$ ", most of us when faced with long multiplication problems such as " 8675 x 7694 " revert to cognitive devices such as pen and paper, or a calculator.

Thus the extended mind hypothesis holds that the organism functions within a modified environment that is constantly being innovated to suit the requirements of problem solving. On this view, extended cognition is an emergent property, whereby there are "no ontological gaps between the different levels of an organism's functioning" (Johnson and Rohrer 2006:24). Thus a critical observation made by researchers who adopt this model is that the adaptive responses that are employed by organisms emerge from the interactive coordination between features of the environment and an organism's patterns of behaviour. Hence an extended mind hypothesis assumes no separation of brain, mind and environment necessarily "for it is always a series of bodily activities immersed in 
the ongoing flow of organism-environment interactions that constitutes experience" (Johnson Rohrer 2006:22). Put simply the mind is embedded within the context of its external knowledge-world whereby culturally transmitted information is an interactive property.

The qualities that emerge from this interaction between the properties of what Clark often calls the 'naked brain', bodily movements and external cultural media. Cognition is not in the cranium but a combination of various biological, ecological, cultural and technological features which he considers to be a 'wideware' cognitive system (Clark 1998; 2001a). The best way to think of it is to imagine a computer with its peripherals. The various additions which we attach to our machine enhance its functionality. It has a 'wider' breadth of capabilities as a result:

"Let us coin a term, 'wideware', to refer to states, structures or processes that satisfy two conditions. First, the item in question must be in some intuitive sense environmental: it must not, at any rate, be realized within the biological brain or the central nervous system. Bodily aspects and motions, as well as truly external items such as notebooks and calculators, thus fit the bill. Second, the item (state, structure, process) must play a functional role as part of an extended cognitive process: a process geared to the promotion of adaptation success via the gathering and use of knowledge and information, and one that loops out in some non-trivial way, so as to include and exploit aspects of the local bodily and environmental setting (Clark 1998:273) 
Merlin Donald (1991) anticipated similar views and has offered a unique perspective on the evolution of hominine cognition that reflects Clark's extended mind model. Crucially, human intelligence according to Donald is only partly attributed to 'brain' evolution. Donald argues that hominines have overcome the cognitive limitations beset by their biological constraints, not by content-rich modules, but by the use of external cultural tools such as symbol-use. Donald recognizes the considerable impact that co-evolutionary processes have had in the cognitive development in hominines (i.e. the co-evolving dynamic between cultural environments and biological properties). Thus he disputes the claim that the evolution of human cultural traits remains the result of the biological preconditions endorsed by EP (whereby the evolution of content-rich cognitive architecture preceded hominine cultural intelligence). Instead cultural properties and hominine cognition have had evolved together in an interdependent, or hybrid process.

Donald argues that hominine representational ability, such as the use of symbols, is in all probability the result of a large-scale "integration within the nervous system, and the integration with the cultural environment" (Donald 1995:1093). He suggests that human intelligence then has the capacity to integrate a wider variety of cognitive domains both internally and externally. Donald argues then that Homo Sapiens appears to possess what he calls a 'hybrid mind'. Much like the notion of scaffolded intelligence, and Clark's extended 
mind approach, the hybrid mind hypothesis rests upon the premise that cultural inventions make us smarter. Both Clark and Donald recognise two additional premises to their models: a) the voluntary control of bodily movement has led to greater breadth of learning and development; and b) our learning environments are constructed in order to support cognitive expertise (Donald 1991, 1995; Clark 1998).

Avital Jablonka and Marion J. Lamb (2005) have also shown that we not only pass on language systems that allow for the communication of representations and referents, but we also inherit a comprehensive symbolic system whereby these discrete representations are "self-referential" within a larger whole (Jablonka and Lamb 2005:199). For instance the "religious" worlds that people inhabit are comprised of context-dependent signs and symbols that are situated within a comprehensive structure of meaningful representations. The imagery associated with the Christian Eucharist with its concept of "flesh" and "blood" as being representative of "spiritual" fulfilment is recognizable as such within larger integrated context. Hence Christians normally interpret these rituals within "a shared framework of religious or artistic practices in which they have a role or function" (Jablonka and Lamb 2005). So for Jablonka and Lamb (2005) symbols aren't just token signifiers, mapped on to a referent. Symbols are context-dependent, or self-referential within a larger system of meanings and signs. Yet they are also actively 
engaged with by agents, whose interaction shape and reproduce these representations. As Jablonka and Lamb point out:

"We can say that signs... become symbols by virtue of being a part of a system in which their meaning is dependent upon both the relations they have to the way objects and actions in the world are experienced by humans, and the relations they have to other signs in the cultural system" (Jablonka and Lamb 2005:200).

Thus the meanings of a symbol can be interpreted in a variety of ways, yet the symbol itself has a place in a broader system of knowledge and culture. A picture of the Crucifix may warrant a different interpretation for a Hindu, yet it is still part of a larger integrated system constituted by other symbols and meanings for Christians. Thus we must careful when making the assumption that there exists fixed a nature both materially and symbolically. Symbols are information certainly, however: "...the process of acquiring information is an active one that involves the reconstruction and transformation of the information" (Jablonka and Lamb 2005:205). On this view, Jablonka and Lamb see symbols as mutable structures. Conceptual reasoning is borne out of engaging with ever-changing external symbolic artefacts. But discrete symbolic representations are dependent the symbolic context which supports the meaning-making process. This symbolic environment is, on their view, inherited from one generation to the next. 


\subsubsection{The Cognitive Niche}

Individuals and societies inherit cultural environments that have been built by previous generations. These environments are subsequently improved upon and then passed down (Tomasello 1999; Sterelny 2003). For instance, technologies from previous generations have benefited from innovative improvements, which are then handed on to the next generation (and so forth). This dynamic, within cultural evolution, as been dubbed by Michael Tomasello as a "ratchet effect" which Tomasello offers as an explanation for the process of cumulative cultural evolution (Tomasello 1999). Cultural invention, on his view, is 'racheted up' with each innovation providing a platform for further innovation and transmission (no matter how small), resulting in the accumulation of information and technology over time. Cultural technologies improve our cognitive abilities - take writing and literacy for instance - and subsequently lead to a greater breadth of skill and expertise. Like Clark's notion of wideware, the niches that humans inhabit seem to be cognitive system as well as an ecological one.

Thus such models seem to support a cognitive niche construction hypotheses (Laland and Odling-Smee 2000a; Sterelny 2003). Traditionally within the biological sciences niche construction has been viewed as a form of "ecological engineering" whereby organisms actively modify their environments for fitness advantages. 
While these changes have noticeable effects on the environment and other species (beaver dams, termite mounds and spiders webs are common examples of this), they also have a fitness advantage if organisms can modify the environment to suit their own needs. Kevin Laland and John Odling-Smee (2000a) have described this process more simply as organisms making a living via a symbiotic relationship with their environments. Thus organisms often: “...choose their own habitats, mates and resources, construct important components of their local environments, such as nests, holes, burrows, paths, webs, dams and chemical environments, and choose, protect and provision "nursery" environments for their offspring" (Laland 2004:316). During these processes the environment is altered, further altering the behavioural patterns of the organisms inhabiting these engineered ecologies.

In addition, subsequent generations are able to take advantage of the ecologically engineered environment built by previous generations. For instance, earthworms despite having anatomy and physiology designed for freshwater environments: "are able to survive in soil by physiology, through activities such as choosing the optimal soil horizon, tunnelling, exuding mucus, eliminating calcite, and dragging leaf litter below ground" (Laland 2004:321; Turner 2000). Earthworms inevitably alter their environment making it habitable for themselves in virtue of their behavioural patterns, while creating a heritable living environment for future generations. Thus niche 
construction processes allow for the non-biological (non-genetic) inheritance of ecologically engineered habitats.

But these niches - particularly in the case of humans - are epistemically engineered environments also (i.e. information-rich, or knowledge environments). Thus while material properties are passed on from generation-to-generation, these material properties often embody valuable informational resources. As Daniel Dennett (2000) has observed, a tool has a two-way function: it is both purposeful in its instrumental value and a blueprint for the manufacture of future tools. Hence a tool acts additionally acts as memory device (Dennett 2000), while language and imitation allows for valuable information to be pass on to downstream generations, such as the case in learning skills.

Indeed, the cognitive niche is an important area of focus in the evolution of hominines. A discernible feature of Homo is their exploitation and use of material culture. The success of early Homo was not necessarily dependent upon fitter genetic traits, but additionally dependent upon our capacity to construct and reconstruct fitter environments. Yet Donald and others (Clark 1997; Donald 1991; Mithen 1996a; Sterelny 2003; Tomasello 1999) infer a connection between external and internal cognitive worlds. Again, a hybrid mind facilitates the cross-networking of cognitive domains (both internal and external). Yet these domains can mutually reinforce the 
development of more sophisticated cognitive traits such as social intelligence (see Sterelny 2003), symbolic abilities (Deacon 1997; Donald 1991), moral decision-making (Clark 1996), or even religious cognition (Day 2004; Mithen 1996a, 1996b).

Learning and development is greatly enhanced by the cognitive properties of our cultural niche. Kim Sterelny (2003) posits that Homo Sapiens do indeed inherit more basic perceptual modules as standard features of our cognitive architecture. However, these modules (previously linked to stereotyped action typical to our primate ancestry [see Cheney and Seyfarth 1992]) operate within a developmental learning environment coactively. And so sensitive is this developmental plasticity to environmental learning that our computationally limited "basic brain" can be enhanced "and ratcheted up to greater precision" (Sterelny 2003:223; See Tomasello 1999). Indeed within the wideware model of cognition human intelligence is not solely composed of genetically entrenched informational resources. On the contrary - hominine intelligence has evolved to a degree whereby our cognitive expertise is supported within a "cognitive niche" comprised of non-genetic informational resources. Thus, the repertoire of skills available to an agent often depends upon a dialectical relationship between brains and environment ${ }^{7}$. Humans

\footnotetext{
${ }^{7}$ By 'dialectical' it is assumed that agents are informed by their social-cultural environments, and by interacting with that information agents thus transform or modify their circumstances - further modifying the epistemic environment of others. Richard Lewontin, et al (1984) describe the dialectical approach to behaviour: "Dialectical explanations... do not abstract properties of parts in isolation from their associations in wholes but see the properties of parts as arising out of their associations. That is, according to the dialectical view, the properties of parts and wholes codetermine each other... It follows, then, that dialectical explanation contrasts with cultural or dualistic modes of explanation that
} 
are unique among primates because our dependence upon sociotechnological resources remains crucial to our survival.

Researchers have inferred then that hominines have co-evolved with the niche-constructed environment they have created. Within coevolutionary models cultural processes and properties are viewed as having a significant selective pressure on the evolution of hominine intelligence. ${ }^{8}$ This alternative view To EP suggests then that culture has played a "co-active" role in the evolution and development of modern anatomical Homo. Paul Griffiths and Karola Stotz (2000) describe a similar co-evolutionary scenario:

\footnotetext{
"... Modern humans owe a good part of their capacity to develop cognitive powers to the successful replication of earlier developmental systems which included a range of social and cultural resources. Humans are born into intentional surrounding as part of a lineage which has coevolved with environments in which intentionality and representation exist in other subjects and in objects and the context-of-use of these objects" [Emphasis added] (Griffiths and Stotz 2000:45)
}

separate the world into different types of phenomena - culture and biology, mind and body - which are to be explained in quite different and non-overlapping ways" (Lewontin et al 1984:11).

${ }^{8}$ The Baldwin effect offers a theoretical approach to ontogenetic biological processes, whereby prolonged behaviour has a noticeable effect on the evolution of certain traits. Instead of genetically entrenched biological features, Baldwinian selection is enabled by more open learning and developmental flexibility whereby new skills are learnt which have a salient effect on the inclusive fitness of organisms. See also, material on Baldwinian evolutionary processes: M. J Baldwin, A New Factor in Evolution. The American Naturalist, Vol. 30, No. 354 (Jun., 1896), 441-451. And also for a good overview of the debate see: K. Sterelny (2004) The Baldwin Effect and Its Significance: A Review of Bruce Weber and David Depew (eds) Evolution and Learning: The Baldwin Effect Reconsidered; MIT Press, Cambridge, Mass 2003, pp x, 341. To appear in: Evolution and Development. 
Cognitive niche construction and co-evolutionary models challenge the view that hominines have been purely passive observers in their own evolution. The inferences surrounding the ancestral history of humans seem to suggest that we have been co-actively shaping the environment to suit our own ends. However, this inherited environment has had noticeable effects upon downstream generations leading to dramatic neuro-physiological changes also.

4.2. The Co-Evolution of Human Intelligence: Evidence from neuropsychology

As I mentioned Merlin Donald (1991; 1995) offers an examination of human cognition within the scope of neuroanatomical research, focusing on the evolution and enlargement of the pre-frontal cortex in hominines. Importantly Donald suggests that EP is underdetermined by the neuropsychological data. Indeed neurophysiologists would expect to find the most recent developments in human cognition (i.e. those modules that made us more intelligent) to be canalized and content-rich. But Donald says this is notably untrue of the human brain:

"The newest parts of the brain do not constitute a fixed instrument whose adult functional arrangement is static and largely innate, but rather a dynamic system in constant flux, with shifting boundaries and malleable internal linkages" (Donald 1995:1093). 
Indeed the most recent neural developments in Homo Sapiens evolution are within the frontal region of the brain (particularly the tertiary cortex), which "by definition less fixed in the functional linkages of inputs and outputs than primary cortical regions" (Donald 1995:1093). Having no immediate functional linkages to these areas, the most recent neo-cortical regions (particularly the tertiary regions) remain functionally plastic. Hence while there may be modular cognitive properties, these modules are hierarchically organized and highly dynamic in their connectivity. According to Donald then this capacity to representational such a way is more than likely due to increased neural plasticity in hominin evolution, not specialised cognitive systems. Instead, our capacity to learn skills through the voluntary coordination of our actions ensures a greater breadth of responses to a variety of cues (Sterelny 2003).

Similarly others such as Kim Sterelny (2003), Clark (1997), Terrence Deacon (1997) and Steven Mithen (1996a; 2001) have endorsed a comparable approach to Donald's. For instance, Sterelny (2003) says that what makes Homo Sapiens an exceptional species is our capacity for "higher-order" thinking - such as the ability to think outside of the immediate context (Sterelny 2003) $)^{9}$. Sterelny observes that humans

\footnotetext{
${ }^{9}$ Through the co-regulated repetition of tasks between teacher and learner, infants begin to adopt the self-regulatory movements required for autonomous, decoupled action (Clark and Grush 1995). Thus, eventually, instead of imitating directly the movements of others, infants become more adept at watching (visual stimuli) and subordinating that visual cue into other motor productions. Thus a child might watch how to kick a ball (input), and then attempt to do the same task by controlling and regulating the bodily movements necessary to achieve this. But the child while learning is constantly monitoring and regulating the output necessary to achieve this imitative task. Once this skill is learned, however, a child can then apply the same movements to other contexts or situations (kicking leaves or sand for instance). Thus the cortical control of movement allows for the higherorder of associative relationships of learning whereby one learned task can be subordinated to
} 
possess the ability to "decouple" cues from stereotyped actions (i.e. actions that aren't typical to single-cue, single responses). For instance, humans are highly flexible when it comes to learning and voluntary movement. The complex processing associated with planning, preparing, and subsequently acting autonomously requires a certain class of information processing effectively disengaged from any immediate cues in the environment. Sterelny calls this capacity to think ahead and act based upon the information provided - "decoupled representations" [Sterelny 2003:95-96]). Our representational ability is thus not cued to any single connection in the world. Instead humans function with a remarkable level of self-control over the motor nervous system.

Stereotyped action within the biological sciences is usually seen as being cued to a particular type of detection system in the animal, thus actions in response to contingencies often remain limited to single-cue / single-response actions (Sterelny 2003). Most herbivores such as sheep or cows are predisposed to evade certain proximate cues such as an approaching movement. Humans, however, make decisions and act upon them based upon the information provided. Subsequently if agents manage to track their world accurately (i.e. generate adaptive decisions) then decoupled representations become, what Sterelny and Peter Godfrey-Smith, call "fuels for success" (Sterelny 2003; Godfrey-Smith 1996). In light of this examination, it is often criticized that the 'fast and automatic' system implied by EP (see 'juke-box' analogy above in 
Chapter 2) is perhaps an insufficient explanation in light of this capacity ${ }^{10}$. Indeed Sterelny proposes that cognitive constraints would be impractical for enabling such cognitive flexibility. Information, plus cognitive flexibility allows for a greater breadth of action. Consequently our cognitive expertise remains dependent upon our learning development.

Donald (1997), Clark (1997) and Deacon (1997) have observed that a significant level of human cognitive development is "activitydependent". That is, learning development is not solely derived from normal/biological maturation processes alone, but by an active engagement with the cognitive world of 'externalised' culture. As a result the modular view of the brain as being comprised of contentrich "mental organs" has been called into question. The cognitive development of human beings is seen now as being shaped by environmental influences, which subsequently affects the structure of our neural-circuitry. Put simply, there is a great deal of interdependence between our functionally 'plastic' neurological resources and the cognitive resources of our cultural environment.

Neuroscientist Steven Quartz (Quartz 1999; Quartz and Sejnowski 1997) has presented similar research (mainly as an alternative view to the EP model of domain-specific intelligence). Instead of the brain as being as massively modular - with sophisticated genetically

\footnotetext{
${ }^{10}$ Although for an update on EP's approach to open developmental programs see: L. Cosmides \& J. Tooby. (2000). Consider the source: The evolution of adaptations for decoupling and metarepresentation. In D. Sperber (ed). Metarepresentations: A multidisciplinary perspective. New York: Oxford University Press.
} 
entrenched traits - Quartz endorses "hierarchical" model of human neural and cognitive development. Quartz argues that despite their being primary sensory and motor cortical areas which mature earlier and are more activity-independent, a large number of neural networks additionally participate in "the postnatal construction of neural circuits" (Quartz 1999:51). Quartz's “constructivist” view of neuropsychological development posits that the brain interacts with the learning environment thus structuring the actual neural circuitry required for learning. This includes the growth and number of synaptic connections, axonal processes and dendritic arbors which become progressively denser as learning development increases. Yet this does not indicate a greater capacity to process informational inputs. What it indicates - according to Quartz - is a kind of developmental/canalization process that embeds neural devices required for learning.

This research agenda is not new. Karmiloff-Smith (1992) has previously endorsed a similar approach to Quartz in which she elaborates on the processes of automated cognitive routines, with modularization being considerably determined by learning development. Karmiloff-Smith suggests that during learning development knowledge becomes applicable for non-specialized purposes across different motor routines. She calls this process 'representational rediscription' whereby cognitive traits while becoming automated and more entrenched overtime, subsequently 
are made available to other task-solving cognitive regions. Indeed learning becomes decoupled from context-dependent situations. For human primate cognition, learning how to carve bone into a spear for instance means that such a skill can potentially be subordinated to other contexts also (e.g. a symbolic context - by applying the same technical skill to carving symbolic representations or stylised motifs).

This suggests to Karmiloff-Smith that we can employ stably learnt skills and establish "perceptual links" to other behavioural outputs. Thus while earlier more primary cortical areas - such as basic sensory and motor areas such as bodily coordination and attention appear to be mature at birth, the protracted length of cortical development, coupled with an extended juvenile dependency, suggests that these areas of the brain develop via epigenetic processes, of which these cortical structures appear to be the most evolutionarily recent addition to the human brain. In addition, the prefrontal cortex (PFC) in particular is the most malleable. The PFC lacks any direct peripheral connections to other cortical regions facilitating the capacity for its integration with other areas of the brain $^{11}$.

Because the prefrontal areas develop later in maturity, the region's dependence upon environmental stimuli suggests its inherent

${ }^{11}$ Earl Miller (2000) sums up the function of the prefrontal cortex well in his review of research into this debated region of the brain: "The prefrontal cortex is an interconnected set of neocortical areas that have a unique, but overlapping, pattern of connectivity with virtually all sensory neocortical and motor systems and a wide range of subcortical structures. This provides an ideal infrastructure for synthesizing the diverse range of information needed for complex behaviour" (Miller 2000:59) 
plasticity. Put simply, the prefrontal cortex is able to accommodate input pathways while being functionally integrative of other cortical areas. As Terrence Deacon (1997) has observed, the prefrontal cortex is potentially larger than the motor and pre-motor cortical areas relative to our body size, meaning that the prefrontal area is far less operationally uniform, and subsequently less determined by primaryperipheral connections (See Quartz 1999:54). Thus humans have developed the capacity to employ a greater breadth of attentional control to areas associated with primary sensory and motor cortical areas.

\subsubsection{Primate Intelligence and the Cognitive Niche}

This process of learning for facilitating other, more complex cognitive skills has been illustrated in research on Bonobo chimpanzees' symbolic associations using lexigram keyboards. In experiments by Savage-Rumbaugh and colleagues (1986; 1994), a chimp named Kanzi and two other chimpanzees were trained in the use of visual lexigrams comprised of visual pictures of objects associated with actual words. What they found was that not only could the chimps pick out symbols on the lexigram keyboard and communicate to the researchers (e.g. "I want food" by pointing to a picture of an apple), but Kanzi in particular was able to hear a spoken word and accurately choose the corresponding symbol on the 
lexigram board. Kanzi's success rate was 100\% without any explicit prompting.

Savage-Rumbaugh and colleagues reported that the cross-modal and sensory-modal integration of auditory cues to symbolic visual correspondences thus were not only limited to the human species, but to bonobo chimps also via cognitive devices. Yet this required a specific type of enculturation similar to the human cognitive niche. Yet the reason why Kanzi was a particularly unique case in comparison to the other chimps (Sherman and Austin) was that Kanzi appeared to have an implicit knowledge of symbol-use at a younger age. SavageRumbaugh et al (1994) attributes this more advanced capacity to Kanzi's implicit training via the mother's lexigram training (who continued to struggle with learning the rules of communication through this tool). Kanzi instead, by just being exposed to his mother's learning at an early age, had a superior facility with symboluse (See Deacon 1997:124-127).

Unusually Kanzi possessed the ability to voluntarily concentrate his attention within the context of communication with the lexigram board, while other chimps had to be told what to pay attention to (Deacon 1997:127). This illustrated Kanzi's ability to cortically control his movements, his intentions and attention to symbolic representations. Thus Kanzi began to use the lexigram board to signal his intention to others, but also use his body as a 
representational device for indicating his intentions (i.e. with gestures and pointing etc). What the Kanzi example shows, however, is that encultured chimps are more capable of imitation than mother-reared chimps (Tomasello, Savage-Rumbaugh and Kruger 1993). Put simply culture can release in Bonobos their "latent communicative potential" (Donald 1995:1097).

\subsubsection{Towards a Co-Evolutionary Model for Hominines}

Despite Kanzi's ability, however, it is improbable that Kanzi would ever develop the capacity to use a complex system of language comparable to humans (Donald 1991:136). Though Kanzi could initiate novel combinations of representations including gesturing, he never went beyond two-word, sometimes three word combinations. Yet Deacon (1997) and Donald (1995) argue that Kanzi's capacity for symbolic behaviour is an indication of proto-language skills, a capability that our Australopithecus ancestors may have possessed. According to Deacon this proto-language ability had almost certainly developed into a more sophisticated system of communication by the time Homo Erectus arrived on the scene (See Deacon 1997:340349).

Thus the accumulative processes associated with cultural transmission fuelled physiological changes in the hominine lineage which inevitably fuelled further cultural development, which in turn 
selected for further cognitive elaboration. Somewhere in our ancestry (Deacon cannot really infer when or how) the transition from Australopithecus to Homo Symbolicus was first initiated by externalized representations and artefacts enabling hominines to cross the threshold from a cultureless species to a cultured species.

The socio-ecological pressures then that led to hominines adopting tools and symbolic representations further selected for neurophysiological changes. Thus as verbal language slowly became a more efficient means for communication selection acted on the cortical control of the mouth, jaw, lips and tongue for speaking including the unusually low descent of the larynx in Homo Sapiens. Deacon (See Deacon 1991:439-365) argues that this has resulted from a co-evolutionary process involving a hybrid progression from non-verbal symbolic behaviour i.e. gestures and bodily control of movements, to eventual vocalization.

Deacon (1997) and Donald (1991) argue that the PFC is essential to symbolic learning and higher-order representations. Likewise both see symbolic behaviour and language as the "prime mover" in a coevolutionary process between the encephalization of the pre-frontal region and the external properties associated with symbolic representation (Deacon 1997:45). Deacon proposes a more Baldwinian process: as symbolic behaviour became more complex, selection acted on regions in the brain that were able to cope better 
with this emerging complexity. Yet this model doesn't view natural selection as acting on a specific region or module in the brain devoted to language acquisition. Instead selection was "cheaper" "dirtier" and more efficient in its utilization of existing neural structures in the brain. Thus selection may not have selected for something as elegant as a language "mental organ" for instance. Instead selection opts for neural plasticity which is crucial in the interaction between agents and the external properties culture (Clark 2001).

\subsection{Summary and Conclusion}

This chapter presented a measure of theoretical challenges to the concepts offered by EP in chapter 2. Merlin Donald suggested that EP needs to readdress some of the correlations between its concept of domain specific systems and the neurophysiological evidence. He stated clearly that the modularity thesis is undetermined by the data. Donald argued that there are strong connection properties which exist linking our internal cognitive machinery and external cognitive resources. And it appears as if our brain has evolved to rely on these resources. Cultural properties not only support or 'scaffold' the computational limitations of our biological brains, but Donald proposes that genes and culture have co-evolved in a mutual interdependence. EP Donald argues, undervalues this integrated dynamic. Further, neurological evidence suggests that the most recent development in hominine cognitive evolution has been in the prefrontal cortex - which is functionally plastic, not content-rich and 
specialized. Thus it is this 'hybrid' integration, between brains and culture, which has determined the evolution of human intelligence.

An examination of the cognitive niche that we inhabit can be considered a 'wideware' model. Cognition isn't limited to the 'space between our ears' (as offered by EP's 'hardware' model). Subsequently cognitive capital shouldn't be viewed as just simply neurological resources alone. The cognitive niche is comprised of a variety of non-genetic resources which supports our intelligence. Our ecologically engineered environment sustains the coordinated and cooperative transmission of information to current and downstream generations for the purpose of learning and innovation. As in the Kanzi example, it is the presence of cognitive tools, coupled with enculturation that established its symbolic ability. Kanzi was raised in a cognitive niche where the transmission of cognitive skills were coordinated and supported by human agents and instituted learning tools and techniques.

The same is true for religious learning. The concepts associated with theological information are difficult to transmit and learn without the use of symbolic-cognitive tools and social learning. Religion - far from being 'natural' - is a culturally transmitted complex. The religious cognitive niche consists of an interwoven complex structured for the transmission of theological knowledge. And as shall be shown in the following two chapters, the religious cognitive niche exploits 
unique features of our cognitive architecture in order to support the transmissive frequency religious representations. Symbolic learning remains a crucial feature of human intelligence, but it is the storage and transmission of these symbols which is so fundamental to cultural inheritance. I will further show that without this form of cultural technology, humans would not be able to develop the complex bodies of theological knowledge and concepts which constitute religious beliefs and practices. 


\section{Chapter 5: The Evolution of Symbolic Storage}

\subsection{Introduction}

A conspicuous feature of human cognition is our capacity to employ and manipulate symbols. Unlike chimpanzees humans are well-equipped to engage socially and communicate using gestures, speech and artefacts. Kanzi was able to respond to cues and employ basic symbolic signs, while humans have the capacity to not only use symbols to pass on information, but also to develop complex 'higher order' symbolic associations such as metaphors and analogy. We also have the capacity to construct densely interwoven symbolic worlds comprised of theological concepts which we feel meaningfully connected to. Symbols of prestige, for instance, differ from culture to culture. There may maybe asymmetrical relationships between religious adherents and the religious elite (e.g. priesthoods, or elders). Yet this status may hold little sway in Western secular societies where you find titles and prestige connected to commercial success. Hierarchy and symbols of prestige evolve out of a particular cultural context.

Religious beliefs and practices are an example of the human capacity to organise and arrange a symbolic system whereby value is assigned to particular objects, places and individuals. But how and why did this come about? How did humans evolve the capacity to 
inhabit this symbolic niche? This chapter will look at the wideware model discussed in the preceding chapter, but focus specifically on the relationship between the evolution of symbolic intelligence and the cognitive niche. Donald's co-evolutionary model for hominine intelligence will be further expanded upon, which divides cognitive evolution into three major transitional periods in our ancestral lineage. On this view it is hominine activity that has determined the development of symbolic intelligence. It has been employment of symbolic communication within a socio-cultural context that has driven hominine cognitive evolution in its unique direction towards complex representational ability.

However, human beings have the ability to manipulate and store symbolic representations in our external environment. While physical gesture and speech have been important steps in the development of Homo-Symbolicus, it is the creation and use of symbolic artefacts which have defined our cultural evolution and thought. Indeed symbolic artefacts, on this view, are seen as 'thinking tools' which support the processing of cognitively difficult concepts. Thus the 'symbolic storage' of representations has supported what Donald calls a 'theoretic culture,' constituted by abstract forms of communication and worldviews. As I will show further in this thesis, theological knowledge not only depends upon symbolic thinking tools for transmission, but the complexity of this knowledge has also 
affected our capacity for thought.

\subsection{Merlin Donald's Three Stages}

Merlin Donald (1991) endorses a triadic process within symbolic learning as a key component to the co-evolution of hominine cognition. Donald proposes that humans have evolved the capacity for learning symbolic associations by way of mimetic, mythic and technology supported culture. According to Donald the engine of human cognitive evolution has been fuelled by the emergence of a set of adaptations for representational ability, but more primarily by voluntary physical coordination, self-awareness within a social context and cognitive flexibility. Donald argues that this has occurred at these three transitional stages in hominine evolution. Donald argues that a co-evolutionary feedback loop between brains and culture has occurred in the development of human representational abilities.

Donald argues that while the mimetic and mythic transitions were the result of major genetic changes in human cognitive evolution, a 'theoretic stage' evolved later and was the result of humans employing non-biological resources (i.e. such as material symbols) in order to support higher representational abilities. For Donald the hominine ability for external symbolic storage enabled individuals to 
modify their environment. ${ }^{12}$ Importantly human beings are not only the product of their environments but have also been a major force in creating the environment that has produced their own representational abilities. Symbolic communication, he argues, has been extended into the environment via the invention of symbolic storage and influenced by a radical shift in enculturation that led to a kind of cognitive-cultural engineering capacity amongst humans (1998:15). With the invention and use of external symbols, Donald argues, humans have been able to cultivate modes of thought that would not normally be possible without this technology. Theoretic skills such as complex mathematical thought or scientific reasoning would be impossible without external symbolic control. I argue later that this theoretic skill and complex conceptual reasoning (enabled by a technologically supported culture) remains crucial to our understanding of religious beliefs and activities.

\subsubsection{Mimetic Culture}

Mimetic skill Donald defines as an evolutionary benchmark in Hominine cognitive development. Once early Homo achieved the capacity for the cortical control of their bodily movement, Hominines adopted the capacity to utilize their bodies as representational devices in a public-social setting (see Donald 1991). What it required

${ }^{12}$ Donald never formally uses this term, nor does he make explicit reference to niche construction. However, Donald's understanding of 'symbolic storage' has obvious correlations with a niche construction model. Similarly, the cognitive niche construction model holds that organisms actively modify their environments in order to enhance cognitive expertise. Donald's model too proposes that the active manipulation of external media more-than-likely first supported the computational limitations of memory (see Donald 1991: Chapter 8). 
however was the voluntary manipulation of their physical presence in the form of gesture and movement. Donald $(1991,1995)$ proposes that mimetic skill made it easier for hominines to manage complex social-coordination problems. By being able to voluntarily coordinate one's action in accordance with the actions of others, groups could respond to a variety of socio-ecological problems that only a coordinated collective could overcome (think about a division of labour i.e. hunting and foraging and the kind of problems faced by organizing these social institutions).

But mimetic skill also laid the foundation for verbal language acquisition and skill. Donald notes that despite the necessary physiological preconditions for language (e.g. the descent of the larynx etc) human language: "still emerges from a rich non-verbal communicative background that is held together by pointing, repetition, imitation, gesture, facial and vocal expression, mutual gaze, body language, mutually-directed attention, self-reminding, and coordinated group expressions" (Donald 1995:1098). Thus it is important to recognize still, despite the neural and physiological requirements for verbal speech, that language's utility and learning emerges from a non-verbal context involving the voluntary and coregulatory structuring of physical/bodily associations.

Yet the structuring of a mental representation, while being relatable via a linguistic interpretation of the experience, is nonetheless non- 
verbal in its retrieval. For Donald the self-triggering or auto-retrieval of memory, which allows for a voluntary access to certain images or representations, maybe derived from the mental rehearsal of a particular experience associated with motor activity, or what Donald calls "mimetic" skill. For example, when someone asks "Describe your bedroom", there is the intuitive conception that a centralized "self" has the capacity to access a representation of the space that is your bedroom. Yet, Donald says this capacity is derived from our “kinematic imagination." Thus: "to conjure up one's bedroom, the best strategy is to imagine the motor activity of 'getting up' or 'walking across the room"' (Donald 1995:1091). Put simply, the 'mental rehearsal' of a particular experience has physical and bodily correlates connected to the representation.

The 'mimetic' recall of an explicit memory is essentially a procedure that is associated with the sensory-motor experience of the body. Prior to being able to express experiences linguistically, hominines first had to acquire the capacity for auto-cuing memory retrieval independently of environmental cues. Thus memory retrieval is autonomously self-organizational in the reconstruction of mental imagery, yet not dependent upon language. Hominines were first able to model their bodily activity both as a physical representation (i.e. imitation or gesturing) and as a mental representation (i.e. memory) before the evolution of complex verbal communication (and it is pertinent to note that mimetic skill facilitated the evolution of the 
meme - or the imitative learning of cultural representations [Dawkins 1976]).

The recoding of knowledge is the human capacity to construct symbolic representations of experience. Thus symbolic representations can produce pathways for the retrieval of memory. So bodily-rehearsals in the form of ritualized-mimetic activity were probably the first forms of symbolic representation. The bodilyrehearsal of a certain experience, for instance, is a purposive endeavour involving repetition and the self-modelling of a conscious representation (Donald 1991, 1993). The body became a source for symbolic representations.

The mental rehearsal of certain activities is derived from the crossmodal perception of the experience (i.e. visual cues say coupled to the cognitive organization of sensory-motor dynamics). Likewise, for Donald, the recalling of certain experiences involves the rehearsal of the sensory-motor activity via mimetic skill. Thus hominines are unique, he says, in their capacity to evoke certain sensory stimuli as representations; to recall and "symbolize" a past action or experience. Thus the neuro-physical composition of hominines is constituted by a highly integrated nervous system that can self-organize and crossnetwork different modes of representations. In symbolic thinking we can self-organise and express a variety of novel representations because of this integration. Metaphorical associations such as: 'It's 
raining cats and dogs' can be employed to describe a particular weather condition.

According to Donald then firstly we had to acquire the capacity for voluntary communication. Publicly represented bodily movement thus constituted the first complex social form of communicative abilities. By controlling the output of physical representations, we were able express meaning through voluntary action-patterns. This, for Donald, illustrates a "supramodal" adaptation for communicative purposes. By supramodal it is assumed that we were able to employ physical movement and action within the realm of higher order associations i.e. deliberate representations endowed with meaning. This mimetic skill capacity would have required the "conscious scrutiny and improvement" of actions (Donald 1995:1096) that only auto-voluntary action can provide. Through this capacity to mimic others' behaviour agents can model actions and assume the intentionality of a representation within the social-public realm.

Thus agents had to have an awareness of "self" as intentional actor yet this only arises out an awareness that the actor itself is a "public representational device" (Donald 1995:1094). Thus the consciously repeated act of a physical representation is "in effect representing itself, to both the actor and the audience" (Donald $1995: 1096)$. Out of this the actor begins to form a representation of self as intentional. Agents become aware via mimetic skill that they 
are a meaning-making apparatus that is constantly being interpreted by others. Thus the cortical control of the physical-representational output becomes crucial to the actor as a result:

"Representational invention on this level raises the question of large-scale integration within the nervous system, and its interaction with cultural environment; once public representations started to evolve, human cognition acquired a culturally driven dimension" (Donald 1995:1093).

Hominines began to inhabit a symbolic world that is transmitted and reinforced culturally. Further, this new dimension of collective communication and representations begin to "transcend" the hominine brain as representations become distributed within the social-cognitive niche. A collectively shared set of representations are thus extended and learned within a cultural milieu.

On this view ritualized activity becomes important for establishing the coordinated activities of a collective (Teske 2001). Bodies can be used as representational devices for social communication while the social environment itself appears as an intentional structure comprised of these representational devices (Dautenhahn 1997). Thus ritual communication becomes an important feature driving the evolution of cooperative strategies. More importantly it also allows for the distribution of shared knowledge within a collective. Human agents have the capacity to "lean-on" the knowledge and skills of 
others, while the transmission of information becomes easier once the voluntary control of bodily movement ensures that people can monitor the outputs of communication and learning. It is also the stage in which signalling behaviour became more complex and representational.

According to Donald then mimetic skill would have enabled the formalization of collective ritual particularly since ritual coordination requires the copying of others' with the intention of simulating a particular behavioural output - for religious purposes or otherwise. Arguably then Donald suggests that the ritualized communication associated with religious custom would have preceded lexical ability and spoken language. Next I will show that the second and third stages of hominine development (both mythic and theoretic) would have been crucial to evolution of the traits associated with religious cognition.

\subsubsection{Mythic Culture}

Donald (1991) suggests that the next step in the evolutionary development of symbolic behaviour came in the form of Mythic culture (see Donald 1991: Chapter 7). The foundation was laid by capacities for the acquisition of lexical skill and speech. Thus while mimetic skill served as a precondition for linguistic competence, hominine lexical ability enabled the capacity to communicate 
representations that were outside the immediate context. With the advent of speech however, individuals could discuss things that weren't in their immediate presence. Thus with the advent of speech we began to transcend our immediate environment by constructing early meaningful associations within a larger discourse.

Donald proposes then that lexical skill and language evolved not only as a social device for communicative purposes, but as means to model reality. With the advent of a semiotic niche there arose a representational capacity used primarily as a means to construct "conceptual 'models' of the human universe" (Donald 1991:213). Thus underlying the structure of language itself, and embedded within a system of symbolic associations, there remains an implicit frame of reference more broadly defined with what Donald calls a mythic understanding of the world:

"The primary objectives of language and speech are thematic; their most salient achievements are discourse and symbolic thought. Words and sentences, lexicons and grammars, would have become necessary evils, tools that had to be invented to achieve this higher representational goal. In this view, language would have represented not an end unto itself but an adaptation that met specific cognitive and cultural needs, that is, ultimately for the formalization of thought and knowledge" (Donald 1991:216).

For Donald (as stated above) the engine in the evolution of language was not the need for social communication. Instead, 
embedded within a system symbolic communication is a model of reality constituted by a semiotic oriented context. For Donald human language systems have a currency within a kind of discursive narrative. Hence it was a need to formulate and conceptually orient a shared frame-of-reference (i.e. a mythic world) that co-actively drove the evolution of symbolic behaviour and lexical invention. Language established a unified mode of thinking about the world within a collectively perpetuated mythic culture. This he suggests is the reason why language systems typically reveal a culturally encoded source of meaning for its users comprised of myths, narratives, beliefs and worldviews that are shared implicitly within the sociocultural context.

This cognitive dialectic between minds and symbolic properties thus constitutes a collectively perpetuated system of beliefs. Yet accepting the structured network of meaning that comprises a social body of shared symbols and knowledge requires a capacity to commit to the reality of those representations. Thus critical to this view are the preconditions necessary for the evolution of a symbolic network; a symbolic network that structures minds and collectives. Chris Knight (1998) quotes archaeologist Philip Chase (1994) when he says:

"Symbolic culture... requires the invention of a whole new kind of things, things that have no existence in the "real" world but exist entirely in the symbolic realm. Examples are concepts such as good and evil, mythical 
inventions such as gods and underworlds, and social constructs such as promises and football games." (See Knight 1998:69).

Linguistic terms, he says, aren't just functional devices that discretely map references to objects in the world. Instead linguistic terms are also set against the backdrop of a more pervasive structure: a communal construct of representations 'established in the universe of discourse' (Knight 1998:69). Hence linguistic terms do not function independently of the network of assumed social discourses constituted by rituals, norms, beliefs and other 'symbolic experiences'.

A symbolic culture is a collective deception according to Knight, as humans engage in 'a world of patent fictions' as a reliable context (Knight 1998:76). Symbols, and their arbitrary relationship to realworld properties, provide us with a frame-of-reference in which to function. Myths and metaphors are appealing while being instructive. Knight argues then that, as symbolic creatures, we adhere to a 'collusion in deception' and the 'maintenance of fictions which have social support' (Knight 1998:76). The human cognitive niche is constituted by a realm of 'collective deceits' (Knight 1995:77 [Sperber 1975:93-5]). Religious beliefs and activities require the complicit support for intangible or 'non-verifiable' representations by all adherents. Thus in order to refer to the representation itself, all 
individuals must 'inhabit the same imaginary world' (Knight 1995:77). Religious systems provide worldviews as a reliable context.

But for Donald and Knight a mythic culture constitutes a socially transparent environment only if individuals are able to communicate within the same fictional world. Interests certainly never converge uniformly. However, they rarely need to if all cultural agents are employing the same communicative resources for representational purposes. On Knight's view, strong reciprocation is assumed as a precondition for communication enabled by the evolution of conspecific signalling. But uniquely, the evolution of hominine signalling is underpinned by an instinctual low-resistant trust of signals. At the outset of his model, Knight cites Krebs and Dawkins (1984), noting that signals in the animal world are frequently high-cost spectacles that are only reliable indicators of an organism's fitness (See Zahavi 1987). They are merely informative in a narrower sense as animals invest a greater level of energy into more elaborate displays for the signal to be recognised.

Subtle signals of communication do not develop especially since readers of signals will not be motivated to risk investing the energy required in interpreting quieter, less costly displays of communication. They avoid such a risk because subtler signals can be plausibly utilized in the deception of others for the signaller's own self-interest (i.e. purposeful epistemic pollution). As a consequence of these 
conflicting interests then animals develop a 'sales-resistance' to these signals, a default position which circumvents potentially coercive signals. Thus, according to Krebs and Dawkins, organisms produce louder displays because (a) they contravene the 'sales resistance' threshold, when they are more conspicuous to the observer, and (b) loud and costly displays are a clear measure of the animal's capacity to meet the fitness costs required for the display.

However Krebs and Dawkins (1984) observe that in cases: "[w]here interests converge... this dynamic is set into reverse" (Knight 1998:71). In exchanges where mutual objectives are fostered amongst conspecifics, there is a greater level of trust and commitment to the signals being shared. Receivers are no-longer constrained by computationally intractable cue-based deductions. Calculating which signal is reliable against those that aren't is a costly process - especially if guided responses generate a higher ratio of false-negatives. As a rule, agents should be predisposed to not cooperate with other agents if the calculative load is too high, relative to the potentially high-cost returns. But the calculative burden of interpretation is lowered if trust or cooperation is tacitly recognized or assumed. As a result signallers need only exert the level of energy required to communicate. Signals needn't be so bold. Thus they become quieter and more concealable. But this also means that signallers can ensure that the energetic burdens generated by highcost displays can be unloaded onto receivers. As receivers are 
motivated to invest more time in the interpretation of signs, due to the expected content of a sign (i.e. signs are assumed to convey valuable information in cooperative social exchanges), then signals become quieter and more nuanced. Krebs and Dawkins calls this communication dynamic 'conspiratorial whispering' (again see Knight for discussion 1998:71-72).

Similarly to Donald, Knight suggests that colluded deceptions are not just the product language abilities, but a prerequisite for language abilities. More broadly however the emergence of a mythic culture in human societies would have enabled: "the production of collective, standardized narratives in mythology and religion, and a narrative frame of governance [for] pre-existing mimetic institutions" (Teske 2001:101). By establishing coordinated activities comprised of customs and routines, cultures could further institute a communal system of knowledge providing meaning and purpose to these activities. Coordination is thus derived from beliefs and practices, much in the same way that Emile Durkheim proposed in his definition of religion, whereby social cohesion is formed through societies organising themselves around the sacred aspects of their culture (Durkheim 1963 / 1915) Mimetic skill allows for simple, episodic representations language and metaphor enables a connection between representations forming an overarching narrative inherent within the context of communication. 
The capacity for myth, on Donald's view, became a means for integrating representations into an easily manageable modelling of reality. Standardized mimetic gestures and rituals could now be merged within a collective network of interwoven symbols. This integration enabled the capacity for small-scale communities to develop a theological understanding of themselves and their world. While religious activities would have been associated previously with rituals that exploited episodic memory events, with the emergence of a mythic culture societies would have been able to embed rituals and customs associated within a communal context constituted by a larger collective of individuals. A society's rituals and customs would thus be underpinned by a sense of relevance and purpose passed-on inter-generationally. And this capacity to endow social relations and activity with meaning would have exerted a greater pressure on memory for transmitting information. A mythic culture and the ability to store this collective information in narratives would have eventually required an easier and more memorable format for communicating a system of beliefs.

\subsubsection{External Storage and Theoretical Culture}

Within the third stage in Donald's co-evolutionary model is the capacity for active-externalism culminating in the "external symbolic storage" of representations (see Donald 1991: Chapter 8). Donald views the external symbolic storage of representations as a crucial 
development in the evolution of hominine cognition. Unlike mimetic and lexical skill which depended primarily upon the individual's capacity for recall, external symbols enabled Homo Sapiens to offload some of the computational burden associated within the processing of cognitively difficult representations. Hence the utilization of external mind tools for Donald is an epistemic act. Artefacts have subsequently become cognitive features that expand the computational capacities of our naked brains. While the ability to model reality through lexical invention enabled mythic culture, the external symbolic storage of cultural models became necessary when representational networks of information became more complex.

It is at this point that Donald suggests that cultures proceeded from mythic structures dependent upon just lexical communication, to a theoretic culture scaffolded by external memory devices. With the utilization of external media or exograms as Donald calls them, societies could engage in the storage of ideas with cognitive technologies further augmenting working memory. Through the capacity for symbolic storage communication became constituted by the offloading of information into the environment. More importantly however the active manipulation of external media enables individuals to direct and organize their thinking.

For example an artist utilises a pen and paper to sketch ideas; to form representations that would not normally be available to the bare 
brain. During the active manipulation of external media agents monitor their outputs and mediate their action in an intentional manner to shape novel representations that were previously unavailable to an individual's brain. Hence language is certainly a prime mover in hominine representational abilities, but it is certainly not sufficient for a theoretic culture. With the active manipulation of external media agents could subsequently produce novel representations thus generating more complex theories and concepts.

In addition, with the advent of external symbolic storage agents could widen, what Donald calls, the 'external memory field' available to them (Donald 1991: 354); further enhancing what may be described as the symbolic invention of cognitive capacities such as conceptual models of reality (i.e. calendars, time measuring devices and texts). Modifiable forms of external media facilitate complex problem-solving and aid in developing possible representational forms outside the limits of the basic brain. As stated, mathematical invention and the development of writing would have not only complemented memory, but widened the theoretical boundaries of what was normally intractable. It could be argued that religious systems depend heavily upon the cognitive technologies required to develop complex theologies and subsequently store knowledge within these symbolic artefacts. 
The modelling of reality facilitated by lexical invention and a mythic culture was subsequently augmented by the capacity to store and modify information in the environment. Robust theological concepts represented in symbolic external media (e.g. alters, statues, paintings etc) can be stored in the environment and act as cognitive properties for religious belief systems directing action and organising modes of conscious experience. Religious adherents, once in the presence of external symbolic media, may utilise an artefact's representational qualities for processing cognitively difficult theological concepts. A theoretic culture scaffolded by cultural technologies such as material symbols can relieve the epistemic burden of processing theological concepts. Indeed the symbolic storage of information may be viewed then as religious 'mind tools' ensuring that the theoretical frameworks represented in a symbolic structure is processed and communicated more effectively.

Donald argues that a crucial feature of human cognition has been the evolved integration between mimetic, narrative and theoretic stages hominine evolution. Thus while these traits have merged successfully, the domains associated with each stage constitutes the cross-networking of information within our cognitive architecture. For instance, the capacity for ritualized communication - in the form of chanting and movement say - indicates a robust interface between mimetic and lexical skill within a mythic context. The body is both a pre-linguistic representational device, while being self-referential 
within the larger cultural narrative associated with the society's belief system.

The network dynamics that support the transmission of religious representations additionally establish a context-dependent structure for individuals and society. Put simply, there is no semantic or somatic content intrinsic to the representation alone. This presents a problem for SM. If all minimally counterintuitive concepts accumulate around innately driven cognitive regularities, then you would expect a greater motivational salience directed towards representations independent of context. What this demonstrates, according to cognitive anthropologist Harvey Whitehouse (see below), is that memory and commitment to certain representations are always "context bound" (Whitehouse 2004:23). Hence the constraints on memory that determines a context are just as significant to a more comprehensive examination of religious belief. However, memory is not bound to the cranium. Similarly to Donald's examination of hominine co-evolution and symbolic storage the constraints on cognition operate in unison (or "dovetail” to use Andy Clark's terminology [1998]) with the external properties of culture.

\subsection{Summary and Conclusion}

We can surmise that the initial concern for the SM was that it didn't really take seriously the properties of culture and their 
influence. The problem for the EP paradigm is that the features of culture are determined by psychological processes first-and-foremost. Likewise, the SM assumes that religious behaviour can be attributed primarily to the preset nature of our cognitive inferences. However, as the above chapter showed, there is a great deal to learn from how environmental factors influence our behaviour. Human agents are not so much disembodied computers as they are 'cybernetic' organisms who depend upon a niche constructed environment rich with cognitive resources (see Clark 2003).

In religious networks, symbolic structures play a crucial role in not only transmitting information (via rituals and myth for instance), but the external cognitive tools utilised in religious systems facilitate a conceptual understanding of a socio-ecology via the use of myths, sacred spaces and social norms. Anthropologists needn't hesitate in assuming that symbols and their meaning constitute a semiotic environment (Geertz 1973), constituted by a "deep web of symbolic relationships" (Deacon 1997:128). Individuals not only inherit genes and learnt information they also inherit entire networks of symbolic information comprised of languages, belief systems, food preferences, writing skills, bad habits and prejudices (Jablonka and Lamb 2005). All these factors make-up a contextual environment that is both intelligible and coherent for agents embedded within these structures. 
So a hybrid mind model may provide an explanation for the problem of theological complexity (re - last section Chapter 3). In other words, it may bring us closer to understanding the topological divergence in religious belief systems cross-culturally. We have seen that individuals are embedded in a cultural-symbolic system constituted by a collectively perpetuated frame-of-reference. This matters because: "All symbolic systems enable the construction of a shared imagined reality" (Jablonka and Lamb 2005:201). Yet the belief in accepting the structured network of meaning that comprises a social body of shared symbols requires a capacity to grasp to the particular context where these representations have meaning (Deacon 1991; Knight 1998).

Humans are not only enduringly cooperative within the cognitive niche (See Sterelny 2003), but the cognitive niche itself is constituted by an engagement with a collectively perpetuated fiction.

Subsequently, the human cognitive niche is constituted by a symbolic reality of collectively agreed upon representations. The reason why religions look the way they do isn't exclusively due to brain function. Instead a causal explanation for theological complexity can be found by examining how a society has niche constructed their environment in relation to socio-ecological circumstances. 


\section{Chapter 6: Whitehouse's Modes Theory}

\subsection{A Study of Religion in "The Round"}

Some researchers have criticized the SM and there has emerged some alternative research within the biological sciences endorsing functionalist approaches to human religiosity (Bulbulia 2004; Bering and Johnson 2005; Sosis 2000; Sosis and Alcorta 2003; Wilson 2002). While authors like Harvey Whitehouse (2004) and Steven Mithen (1996a; 1996b; 2001), have slowly gathered support for (what I would consider to be) distributed cognition models. Whitehouse in particular appears dissatisfied with the SM's research focus. Whitehouse $(2004 ; 2005)$ concedes that the SM might be able to explain the basic cognitive biases for religion (underpinned by minimally counterintuitive concepts, for example) it remains unable to explain the extent to which religious belief systems exert an intense cognitive demand on believers to learn and adhere to rather complex theological systems of belief. Moreover religions are often comprised of highly complex socio-political structures constituted by hierarchical arrangements and social norms. Indeed, because religions are often highly complex there are subsequently many cognitive and physical costs involved with being "religious".

According to Whitehouse the cognitive optimality thesis, endorsed by the SM, "casts its net rather widely" (Whitehouse 2004:45). If 
religious representations were indeed cognitively optimal then why is it that societies tend to perpetuate the demanding practices associated with their religious practices? Thus Whitehouse argues that the SM renders a rather rudimentary "folk" conception of religion - constituted primarily by "supernatural" representations - while further excluding the range of properties that inevitably determine the complexity and subsequently the costs of religious behaviour. For Whitehouse the complexity and costs problem reflects not just the limitations in the SM's definition of religion, but also their understanding of the cognitive preconditions that necessitate the transmission of a culture's belief system. Whitehouse argues that there has to be alternative model of mind proposed before we can offer a suitable explanation for religious cognition. The disembodied computer on this count cannot be enough. Indeed on Whitehouse's view, culture is not evoked. Instead, cultural properties influence and support religious behaviour.

However Whitehouse is an anthropologist, so perhaps he can be forgiven for his tentative criticisms of the SM and its rather "wide" methodological net. Most anthropologists adopt a comparative approach to ethnography. An ethnographer's observation of a culture - and the effort that is required for interpretation - is seldom an easy process. Those who choose to participate in a society's practices encounter difficulties in explaining those "webs of significance" inherent within any system of symbolic behaviour, such as the subtle 
and meaningful interactions between agents and symbols (Geertz 1973). The range of superficial variation that exists cannot be accounted for via explanatory models alone. Ethnography is inherently an interpretive process.

Whitehouse however concedes that the cognitive sciences still has something valuable to contribute to the anthropological discipline. Like Dan Sperber (1985, 1996; see Chapter 2) Whitehouse recognizes that anthropologists often overlook the regularities that emerge within cultures due to constraints of cognition. Yet like any scientific method Whitehouse concedes that the cognitive science of religion should nevertheless attempt to "carve up" the subject matter at the joints, thus laying bare "the mechanisms that shape religious thinking" (Whitehouse 2004:15). Indeed one cannot establish an explanatory model without a system of measurable processes. Ethnographic research is a hermeneutic process, whereby signs and symbols should be interpreted from the standpoint of the cultural actor (or an understanding of culture as a semiotic environment) [Geertz 1973]). However, cognitive scientists recognize that the anthropological interpretation of "meaning" and symbols doesn't offer an explanation for the causal mechanisms that produce cultural representations.

Whereas Boyer would argue that the interpretation of meaning is a trivial problem. Ethnographic interpretations should be deemed a 
separate division of labor from the cognitive sciences, thus it should employ different methodologies. On Boyer's view the cognitive sciences ought to remain an explanatory enterprise. Yet Whitehouse believes that both ethnography and the cognitive sciences combined provide satisfactory explanations for human religiosity. By reconciling two methodologies - both interpretive and explanatory models researchers may achieve what Whitehouse calls the study of "religion in the round". Researchers are able to achieve a more comprehensive model for the study of religion within the cognitive sciences (Whitehouse 2004). Whitehouse believes that the cognitive science of religion can develop an explanatory model that is both widely applicable while being open to the diversity of cultural properties observed in the ethnographic literature. Whitehouse recognizes that while examining the "micro-dynamics" of religious cognition researchers should not by default discard the "macrodynamics" of religious cognition either (See Day 2005: 97 ). Put simply, researchers should not ignore the socio-ecological circumstances into which agents are embedded.

Similar to the wideware model of cognition, Whitehouse highlights the explanatory limits of studying the "isolated mind" disembodied from the non-genetic resources that constitute patterns of behaviour. For instance, Whitehouse suggests that religious cognitive properties can be widely distributed to varying degrees constituting a collective memory within a population. For example, a stratified theological 
hierarchy comprised of the laity up to an elite body of individuals can act as a collective memory store whereby knowledge is secured at differing levels of organization. Access to theological resources may vary (e.g. between the laity and the elite), but those resources including the cognitive system itself - are nevertheless transmittable within a population and across generations.

\subsubsection{Open versus Closed behavioural programs}

Whitehouse (2005) observes that there are currently two types of theoretical positions within the cognitive science of religion, of both open and closed behavioural programs (See Whitehouse 2005:207 for discussion). Closed behavioural programs may be defined - by what nativists classify - as innately-driven cognitive traits intrinsic to human behaviour. Hence, the behavioural outputs of closed cognitive traits are usually viewed as universal and existing independent of context. Whitehouse identifies closed behavioural programs as involuntary responses to external stimuli (such as flinching, laughing and crying), which are uniformly evoked and comparable crossculturally (Whitehouse 2005: 207-208).

Subsequently, it takes precious little ethnographic research to conclude that laughter and flinching "looks" the same from one society to the next. Contrastingly, Whitehouse (2005) views open behavioural programs as hybrid learning processes constituted by 
brains and culture. Thus language, while appearing to have some remarkable similarities in structure and the manner in which it is learned, is nevertheless expressed in variety of different ways. For Whitehouse then, language exhibits not just "open" and "closed" behavioural programs, but "varying degrees of openness" required for its expression (Whitehouse 2005:207). While a flinch or laugh looks remarkably similar, it is the arbitrary relationship that symbols have to their referents that constitute their 'varying degrees of openness' cross-culturally (Whitehouse 2005).

Whitehouse argues that the SM has been in the former camp of closed behavioural programs. Hence, while religious belief systems vary cross-culturally within the SM the primary concern is examining the cognitive regularities of religious representations within human culture. Thus there is no need to examine the differences between the symbolic structures of society - or why one society believes in a certain god over others. Indeed the SM has to varying degrees attempted to explain the uniformity of expression in religious belief. On this view there is subsequently a reducible catalogue of "gods" underlying religious belief systems. Thus if ethnographers only scratch the surface of religious and cultural complexity they will find that supernatural concepts are remarkably limited - or closed - in their expression. The SM suggests then that despite the apparent differences within religious beliefs and practices, external/cultural structures are only capable of doing just one job: evoking a limited 
catalogue of representations.

According to Whitehouse, however, the study of religion should be viewed as a distributed system of internal and external cognitive properties constituted by a complex range of variables which inevitably places mnemonic demands on its adherents ${ }^{13}$. On Whitehouse's view the transmission of religious representations is often cognitively difficult - not cognitively optimal. As a result of this complexity, Whitehouse argues that religious belief systems often employ socio-technological resources in order to support transmission (i.e. external cultural properties such as rituals, texts, symbols, alters, hierarchies, and social norms etc).

On Whitehouse's view religious belief systems have been successfully transmitted inter and intra-generationally as humans have been able to modify their socio-ecological environments in order to facilitate the transmission of cognitively difficult theologies. Indeed religious agents have actively constructed a religious niche in order to exploit memory constraints. Whitehouse suggest then that the culturally transmitted tools utilized by religious belief systems (i.e. symbols, rituals, texts etc) can sufficiently support the cognitive limitations beset by memory. Consequently socio-technological

${ }^{13}$ Please note that the term 'distributed' cognitive system is never explicitly utilised by Whitehouse, yet Matthew Day interprets Whitehouse's model as such in: Matthew Day "Rethinking Naturalness: The Modes of Religiosity and Religion in the Round," In Harvey Whitehouse and Robert N. McCauley, eds., Mind and Religion: Psychological and Cognitive Foundations of Religion, 2002: 207-232. 
resources can indeed support the transmission of cultural representations which do not conform to the cognitive optimum.

According to Whitehouse then if the memorability of religious representations was dependent upon the cognitive optimum alone then the body of knowledge that constitutes the richness and complexity of religious systems would be too unstable due to the mnemonic frailty of individual minds. Individual minds contribute to a division of labor in the construction and reconstruction of a religious cognitive niche. Yet the stability of transmission according to Whitehouse crucially depends upon the socio-technological properties inherent in the cultural environment. Societies relieve the cognitive burden of memory and transmission by establishing networks and materials that scaffold this process (see discussion on the cognitive niche and scaffolding above). Cultures and belief systems look different precisely because societies are beset by a range variables and constraints, which inevitably determine the topological features of their religious niche.

"The key cannot be found in the cognitive apparatus taken out of its context of operation, because variables cannot be explained in terms of contents. But a solution may emerge if we take into consideration variations in transmissive frequency" (Whitehouse 2004: 23-24).

Whitehouse's concerns echo the same concerns motivating the niche construction model, that: (a) the variability in religious belief 
systems requires an explanation. Hence researchers must attempt to explain why theologically complex systems of knowledge - that normally depart from the cognitive optimum - are important to cultures; and (b) researchers must also account for why religions depend so much on socio-technological properties of culture for transmission. Similar to the researchers examined above within the niche construction model of human cognition, Whitehouse concedes that the religious mind is enabled not only by "varying degrees of openess" (see overview above), but also by socio-ecological engineering as well.

Indeed for Whitehouse religion is a collective phenomenon. The properties associated with any particular belief system will depend upon collective practices for its successful transmission. Religious cognition for Whitehouse is a distributed system that functions and is facilitated by the external properties and processes of culture (Whitehouse 2005:16). And while Whitehouse doesn't deny the cognitive optimum, he does however propose that religion is a 'hybrid' learning process constituted by varying degrees of openness. Hence while the cognitive optimum exerts a rather powerful gravitational pull towards more intuitive concepts, societies nevertheless value and consequently employ more complex bodies of religious knowledge. 
Because these two dimensions of knowledge remain in competition the more complex bodies of knowledge require different modes of transmission in order to gain a selective advantage over the more 'cognitively optimal' representations. Hence a significant according to Whitehouse is that memory constrains the transmission of complex theologies. Indeed the constraints which define the cognitive optimum will inevitably limit the memorability of certain concepts being transmitted.

Hence Whitehouse recognizes that societies utilize more distributed methods of transmission (comprised of rituals, hierarchies, political systems and material artefacts) in order to stabilize the transmissive frequency religious representations. Indeed memory and the transmission of religious representations can be made more manageable if social arrangements utilize cognitive technologies in order to preserve information to current and future generations. Thus a religious cultural environment could be viewed as a distributed cognitive system (Day 2005).

Yet another problem that Whitehouse recognizes is that the SM often assumes that explicit religious representations are invariably the product of more implicit motivations (see Whitehouse 2004:2426). The SM argues that it is primarily the underlying cognitive 
machinery which motivates individuals to adopt their belief in the concept. However Whitehouse argues that people are "highly mindful" of their professed attitudes within specific contexts. It seems then according to Whitehouse that "explicit beliefs can provide a highly reliable guide to motivation" (Whitehouse 2004:25). Further, he notes that religious belief systems are often comprised of rituals and beliefs that conflict with intuitive processes. Religious adherents spend a significant level of time and energy engaging in costly practices which run counter to individual optimal behaviour (See Sosis and Alcorta 2003; Bulbulia 2004).

As Whitehouse points out religious practices often "override intuitive, implicit inference... and apply explicitly formulated (often massively counterintuitive) principles in their stead" (Whitehouse 2004:25). An undeniable feature of religious belief systems is that they are often comprised of rituals, beliefs and social norms that normally runs counter to the "ordinary thoughts and urges" of its practitioners (Whitehouse 2004:25). Thus Whitehouse argues that if religion is a "natural" phenomenon then why do people do "unnatural" things in the name of minimally counterintuitive concepts? It seems instead that a society's religion exerts a great deal of costs centered on maximally counterintuitive beliefs and practices.

Whitehouse shows that the problems associated with memory and costs can be explained by recognizing two modes in which religious 
representations that are "difficult to remember" and "difficult to do" are transmitted within a culture. Societies solve the problem of memory via costs to ensure that the cultural transmission of religious belief systems remain stable over time. Hence Whitehouse identifies both doctrinal and imagistic modes of religiosity as crucial to the successful transmission of the religious cognitive niche. Although Whitehouse attempts to outline an evolutionary model for the transmissive frequency of religious representations, he does not provide a sufficient causal explanation for how human religiosity emerged. Whitehouse instead is primarily interested in how agents interact with the socio-ecological constraints of a cultural milieu and how religious systems are shaped by these contingencies.

\subsubsection{Doctrinal Mode}

The doctrinal mode can be summed up as ritualized behaviours constituted by high frequency practices (i.e. regular and rehearsed) that illicit low arousal responses (i.e. minimal emotional stimulation). Doctrinal ritual could be viewed as being a component of the sociopolitical features of a religious belief system comprised, for example, by social norms in relation to the religious creed. Ritual in the doctrinal mode thus depends upon the gradual learning of semantically encoded facts that accumulate around socially sanctioned knowledge: 
Doctrinal mode rituals - semantic memory: Whitehouse argues that repeated instances or routines get stored in semantic memory further entrenching automated behavioural routines. Thus semantic memory refers to general conceptual knowledge, yet not specific autobiographical memories (or specific instances). Upon reflection, religious representations that emerge from semantic memory stores remain tacit to the believer. Agents are usually unable to discern the causal factors for semantically determined memories. Indeed semantic memories are usually constituted by motor routines that are implicit to a believer and are more than likely the product of years of enculturation and development (i.e. tacit knowledge such as riding a bicycle).

\section{Doctrinal mode - cultural technologies: Whitehouse} observes that the doctrinal mode is predictably the product of conditioned behaviour. Yet socio-ecological features inevitably determine whether or not society adopts more predominantly doctrinal modes over imagistic modes (see below). Large-scale communities that are difficult to manage employ highly politicized institutions comprised of hierarchical relationships. In addition large-scale communities present a problem for the transmission of religious belief within a population as representations become dispersed within a larger population there is a risk that the cultural variants associated with a belief system will become lost or diluted. Yet correspondingly populations that are big enough to retain a division of labor have at their disposal better socio-technological resources in which to secure and transmit more complex bodies of knowledge. Institutionalization (hierarchies, social norms etc), plus symbolic priming (i.e. symbolic markers, texts, rituals and myth etc), thus produces the likelihood that a 
society will establish more doctrinal forms of religious transmission.

\subsubsection{Imagistic Mode}

Imagistic modes describes low frequency rituals (i.e. irregular and uncommon) which illicit high arousal responses. A good example of this can be rites of initiation which often utilize "shock" tactics such as tattooing or ritual scarring in order to render the experience memorable and salient to the ritual participant. Imagistic rituals maybe comprised of high pageantry, or traumatic acts, that are organized to provide vivid autobiographical memories:

Imagistic Mode - episodic memory: episodic memory consists of distinct moments of experience. These experiences are usually arousing and personally significant to adherents constituted by "vivid and enduring" incidents in a person's life (Whitehouse 2005:211). These moments typically stand out as unique - such as the day a person got married - and because they are often infrequent they depend upon the concentrated arousal that comes from emotionally salient rituals. Hence religious knowledge is embedded via the exploitation of episodic memory; this mode of learning religion is like touching a hot stove - it is salient to individual cognition and infrequently performed. Thus imagistic rituals exploit episodic memory experiences which are usually rich in sensory detail.

Imagistic Modes - non-centralized traditions:

Whitehouse observes that ethnographers are more than 
likely to find imagistic modes of ritual in small-scale communities that depend less upon institutionalized sociopolitical arrangements for their functioning.

Whitehouse argues however that both the above modes should be better understood as extra-genetic basins of attraction "around which ritual actions and associated religious beliefs cumulatively tend to congregate" (Whitehouse 2004:213). Subsequently, religious beliefs and practices which do not conform to these modes will typically become selected out. Indeed Whitehouse argues that the basin of attraction defined by the SM is insufficient for understanding how it is that societies manage the complexity of religious knowledge. Certainly inference engines create a basin of attraction. However, Whitehouse argues that religions are not constituted by just supernatural concepts alone. Instead the cognitive load in having to adhere to a particular religious belief a specific socio-ecological context requires more computational effort (especially on memory) than the advocates of the SM acknowledge. Whitehouse's solution to this problem is to argue that the two modes described above can act separately - or in combination - in order to scaffold individual memory constraints.

\subsection{Socio-Ecological Engineering and the Modes theory}

Viewing the 'cognitive processor' then as being embedded within the socio-ecological context, the human cognitive niche should be 
viewed as a causally relevant feature for the cognitive science of religion. Predictably then, a religious cognitive niche should successfully employ socio-cultural properties which facilitate the frequency of certain representations thus reinforcing the "relationship between inputs and memory effects" (Whitehouse 2004:22). By conforming to the majority of representations agents' memories remain not only distributed throughout a population, but also gradually conditioned by their context. Thus the fast and frugal rule - "copy the majority" - remains a powerful mechanism in the evolution and development of culturally determined behaviour (see Boyd and Richerson 1985).

Notice that this mode of transmission breaches memory constraints. Though the doctrinal mode of transmission remains dependent upon a social learning history and frequent repetition, external guides are also distributed within the environment which act as tacit cues for action. For example, a ritual doesn't have to be stored in individual memory if an agent can attend to the ritual cues within a community of adherents. The epistemic burden of recall then is essentially offloaded into the social-cultural environment. For example, the members of a congregation need not draw upon explicit memory stores in order to "remember" when and how to behave during a service if others' are also repeating the ritualized activity. 
Further, the exegetical reflection of religious teachings - or explicit religious concepts - is determined by an agent's social learning history within a particular cultural setting. However, the use of a religious vocabulary or ritual within a specific context requires that agents share a similar frame of reference for communicating theological concepts. For instance, these can be learned via frequent repetition, or monitored by orthodoxy checks, whereby elite members within a religious hierarchy guide and regulate certain patterns of behaviour (i.e. how to behave accordingly within a sacred context). Within a socio-political arrangement, orthodoxy checks can be regulated not just by leaders within a community but also by other members of the laity.

For instance, folk-level monitoring such as scrutiny by individuals within a small-scale community - or even neighborly gossip - can be a powerful policing tool if the laity fears retribution for not monitoring the behaviour of others (especially if supernatural rewards are deemed an important upshot to ratting out your neighbor for practicing witchcraft). Thus by acting in accordance with majority, socio-political arrangements can effectively reinforce the frequency of representations distributed within a population. Yet because imagistic modes of ritual are rarely performed the prediction is that communities with little or no socio-political arrangements will depend upon rituals that are of high-arousal. As a result, small-scale communities which depend upon imagistic modes will tend to be 
exclusive in nature. This additionally requires that a small, cohesive unit of individuals with exclusive membership is established.

Anthropologists recognize the causal significance that cultural practices play in the patterns of human behaviour, while cognitive scientists endorse minds as being more important to an understanding of human behaviour cross-culturally. However Whitehouse views cognitive processes as being extended into the external environment whereby cognitive resources are enabled by cultural transmission. Much like Merlin Donald's understanding of the hybrid mind (see above), and Andy Clark's wideware model (see Chapter 4; Clark 1998; 2001a), Whitehouse does not assume that the evolution of human intelligence and behaviour stems purely from biological endowments.

Recall that the niche-construction model implies that individuals not only engineer their local ecology, but that they also shape their selective environment in a social-cultural sense. Thus social and cultural constraints, such as institutions and social norms, also select for individual behaviour (or at least conditions certain behaviours). Though this does not imply genetic changes or the development of biologically endowed cognitive abilities, it does imply however a greater breadth of knowledge. Information-rich niche environments develop methods for information transmission. Individuals have to learn ecological strategies; build sophisticated weapons, tools and 
shelter; while also navigating an increasingly complex social world constituted by customs, hierarchies and institutions.

What a cooperative society requires however is transparent information about how a community is socially organized in order to solve coordination problems brought about by a division of labor and its management. Agents need to recognize immediately those prearranged expectations constrained by social conventions, while accurately anticipating the behaviour of others. A symbolic system of shared representations ensures that actions are regulated and lower the ‘calculative burden of strong reciprocation' (Sterelny 2007:722). Thus agents imbue these collectively reinforced symbolic systems with a normative value which 'disambiguate a social environment' (Sterelny 2007:722). Behaviour and action once regulated by normgoverned systems of communication, effectively makes public those mutual requirements for cooperation. Entrenched protocols, customs and rituals make violating norm-governed cooperative exchanges more conspicuous and easier to monitor.

So how do these factors affect the development of socialorganizational patterns and symbolic behaviour in particular? Socioecological demands placed upon complex or information-rich societies would have required (a) a division of labor leading specialized technologies in resource extraction and production (b) more efficient means for communication across not only horizontally 
complex associations [i.e. between individuals], but also vertically complex associations i.e. proto-institutions resulting from a division of labor and larger populations (See Sterelny 2007). And consequently (c) highly regulated patterns of behaviour for reducing the cognitive demands for managing social exchanges. The structure of a society is founded upon a network of social, ecological and economic factors. Whitehouse recognizes then that human religiosity is integrated within these socio-ecological features within a society. Religious customs govern economic behaviour and political structures as well as social exchanges.

Literature on the evolution of religious cognition has focused broadly on the social conditions which sustain religiously defined representations. In certain examples religious information is transmitted or evoked to solve social-exchange problems (Irons 2001; Wilson 2002; Sosis 2003; Bulbulia 2004; Bering and Johnson 2005). And even outside these functional explanations we find 'spandrelist' accounts for such traits subsuming intuitive ontological reasoning about agency (Boyer 1994; Mithen 1996a; see Chapter 3). Not to say that these positions aren't compatible with a niche construction model. But they overlook the importance of social organization brought about by ecological demands. A central feature of religious systems is the collectively prescribed norms that govern behaviour and social conventions. Whitehouse argues that norms of behaviour establish a common language within the social world of 
adherents. Thus, on Whitehouse's view, if social organization is a response to ecological pressures, then one may also infer that normative cultural practices (such as religious norms) were established as a response to socio-ecological complexity.

Whitehouse has argued that the doctrinal mode is not constituted solely by supernatural concepts, but additionally religious belief systems are founded primarily upon a shared system of beliefs, practices and norms. On Whitehouse's account, the social organization and structure of a society constrains the network pathways of information that is collectively reinforced within a community. Information is ritually communicated and reinforced by a network of collectively understood norms and practices. A hunter maybe delimited to a certain position in within a community, but he is also symbolically recognized for his level of expertise. Likewise, individuals are conditioned to recognize positions of authority within their cultural milieu. Individuals, groups and sub-groups assume a recognized status within a social system. Whitehouse's doctrinal mode is defined by normative religious codes structured within a symbolic network of communication. And while the archaeological evidence is scarce at best, particularly for tracking the emergence of religious behaviour, Whitehouse has established a model in which to measure the material and cognitive constraints which shape the topology of religious belief systems. 
Again Whitehouse has touched on some significant obstacles for the SM. But he has yet to establish a causal explanation for why religious systems develop along the line that they do. In other words, Whitehouse has failed to explain how psychological factors interact sufficiently with socio-political arrangements (Pyysiäinen 2006). How are these rather complex theological concepts represented in the minds of individuals? It maybe what llkka Pyysiäinen (2006) calls a "theological fallacy" to assume that a belief system is "more or less accurately reflected" in the minds of individuals (Pyysiäinen 2006). Indeed Whitehouse highlights the constraints on memory and the cultural properties which enable religious belief systems. However little is discussed on the representational content of religious concepts.

At this juncture it would be sensible to review some of the conceptual issues which EP attempts to highlight in its explanation for human cognition and behaviour. Certainly our perceptual systems will inevitably constrain how we register the world, and the SM argues that this will almost certainly be true in the case of religious information. On the SM view, religion looks the way it does because of the kinds brains we have. But what kinds of brains do we have exactly? If we can agree with the cognitivist school of thought that the brain is a representational device - an information processor if you will - then the SM's account for religion is accurate. However if it is more relevant to view human cognition as not just an internal 
process, but as a hybrid dynamic that integrates both internal and external properties, then the SM offers only a partial explanation for religious cognition.

As Sterelny (2003) notes: why assume a "single connection property" between the informational character of the environment and how and agent represents that information, when there are (most notably of human primates) a medley of connection properties both innate and learned which help agents track the world reliably (Sterelny 2003:17; See also Chapter 11.5). Indeed on this view our perceptual systems have been shaped by an inherited epistemic environment. How agents register the world then depends upon not just on internal cognitive processes alone, but also on the informational resources that support the development of our cognitive expertise in a co-evolutionary dynamic between brains and culture.

\subsection{Conclusion: Whitehouse and the Standard Model}

Whitehouse observes that religions around the world often differentiate between cognitively optimal concepts, and "teachings and revelations that carry a heavier conceptual load" (Whitehouse 2004; 2005 Barrett 1999; Slone 2004). In fact, a lot of religious traditions embrace concepts that vary in complexity and depart significantly from the cognitive optimum. Theologically-rich beliefs and practices are conceptually difficult in comparison to the "closed" 
behavioural understanding of god concepts. Hence Whitehouse suggests that the transmission of explicit religious representations cannot be explained by the cognitive constraints that generate simpler supernatural concepts. As Sperber (1996) has pointed out, often symbolic representations are comprised of multi-layered representations dependent upon both dissimilar beliefs/mental states operating in unison, coupled with an interwoven understanding or interpretation of these integrated representations (i.e. metarepresentations).

More significantly however, if the cognitive optimum is the only basin of attraction then the real "elephant in the room" requires an explanation: why the costs of religion? (Atran 2002; Bulbulia 2004; Whitehouse 2004; Dennett 2006). Why do people exert incredible about of time and energy learning these conceptually difficult theological concepts? If the cognitive optimum is correct then it has a long way to go towards explaining why people commit so heavily to certain religious representations with societies investing so much time and resources in the transmission of cognitively costly beliefs.

If the SM consistently utilizes the representationalist paradigm, it is missing something in its examination of human culture and religion. This is the primary concern that Whitehouse has with the SM: their neglect in recognizing the socio-ecological context and the cognitive properties of culture. Whitehouse has recognized that whether a 
society employs ritual modes (imagistic or doctrinal) will depend more upon socio-ecological constraints in addition to the cognitive constraints that the SM underscores as being more efficacious to religious belief. Hence there is an implicit correlation between Whitehouse's modes theory and the niche construction model. Both Whitehouse and niche construction highlight a circuitous dynamic between material constraints (organisms and physical conditions) and culturally evolved traits and conditions.

Whitehouse highlights three significant points in his examination of religious behaviour. Firstly, context matters. Cultural properties should not be viewed merely as an expression of the regularities of religious cognition, but a causally relevant feature in an explanation for religious behaviour. Thus, an explanation for the diversity of religions may be found by looking at the socio-technological factors which support the transmission of beliefs and practices. Secondly, the transmission of religious beliefs and practices involves memory demands and costly rituals. This, Whitehouse argues, maybe difficult to reconcile with the cognitive optimality thesis (constituted by minimally counterintuitive beliefs and practices). Instead theological information is often maximally counterintuitive involving physical and cognitive demands.

For Whitehouse then memory and costs are both the cause and solution to theological complexity. Thirdly, Whitehouse suggests that 
an underlying theme within religious belief systems is that societies tend to adopt beliefs and practices that congregate around either imagistic or doctrinal modes of transmission. Though, cultures often utilise a combination of the two ritual modes, some societies will tend to utilise one more than the other depending on the variables inherent within the socio-ecological environment (see above). Whitehouse supports an extended mind model for religious cognition. The transmission of religious artefacts, practices, institutions and information remains subject to external/cultural contingencies, not just internal/psychological regularities. 


\section{Chapter 7: Towards a Wideware Model of Religious Cognition}

\subsection{Introduction}

I have argued that religious information is not just processed and transmitted via internal cognitive systems, but religious information is also stored and communicated externally. Firstly, human cognition depends upon the non-neurological resources within our cognitive niche. Consequently, human environments are modified in such a way as to preserve religious beliefs and practices (the external cognitive tools which make us 'smarter' also support the transmission of religious beliefs and practices). Secondly, if the information-load in religious transmission was low then the epidemiological theory for the spread of supernatural concepts offered by the SM would be true. However, the processing of religious information is far from effortless. The theological concepts which support the content of religious ideas are highly complex and costly to transmit. The following will show that these two strands mutually support a wideware model for religious cognition. This implies that the external world as a cognitive system functions in conjunction with individual brains to support human religiosity (Clark 1998; 2001a).

In exploring this connection I review the work of archaeologist Steven Mithen (1996) who has made similar observations. Mithen 
argues that material culture not as just an adaptation to ecological pressures but as a form of 'cognitive technology'. A tool can indeed be utilized in the utilitarian sense (e.g. for extracting resources), but it is also carries valuable information in its construction. A tool is utilitarian in the sense that it can act as a blueprint for others' to copy and manufacture other devices. However, a utilitarian device can also be used in a non-utilitarian sense to denote an individual's prestige within a group. As a symbol, a valuable tool may gain a social currency also. Humans he argues are unique because they can cross-network these domains. The technological (i.e. a tool for instance) can become a social device. What is valuable in one domain, can be valuable in another.

Mithen notices that religious belief systems are often comprised of non-utilitarian material items. In addition, Mithen argues that supernatural concepts are too computationally difficult to transmit and process without the cultural evolution of the use of symbolic artefacts. Religious concepts, he argues, remains highly dependent upon a material representation for transmission. Religion is not in the brain. Instead it is enabled by external cultural technologies. Mithen argues that it is 'cognitive fluidity' which has allowed for our capacity to conceive of the arcane beliefs and practices found within religious systems (Mithen 1996:217). The 'fluid' interface between intrainternal and inter-external cognitive domains has facilitated the 
creative development of our symbolic intelligence and consequently religious cognition.

\subsubsection{Offline Reasoning and Non-Intuitive Worlds}

In Chapter 3 I reviewed the experiments conducted by Justin Barrett and Frank C. Keil which showed that individuals could rationalize theological concepts explicitly, but always employ default inferences implicitly while doing so (Barrett and Keil 1996). Though religious adherents could indeed explain that god was omniscient, during experiments where they had to recount a narrative by memory god had a fixed temporal and physical location. Barrett has suggested then that there exists two contrasting yet corresponding psychological kinds operate in unison when reasoning about religious concepts (Barrett and Keil 1996; Barrett 1999; 2000). He argues that people adopt theologically correct positions when reasoning on an explicit-level about their beliefs offline (e.g. when communicating god concepts as a public representation with others). Thus a theologically correct position would pertain to circumstances where individuals justify or attempt to rationalize their religious belief (e.g. why they have faith, or why perform a particular ritual etc). Offline reasoning assumes that a cognitive system can form representations decoupled from the immediate context, or disengaged from the here-and-now. 
In Barrett's words supernatural concepts "are conceptualized on at least two different levels: the basic, everyday concept used in realtime processing of information, and the theological level used in discussion of God's properties or activities outside of on-line, realtime processing demands" (Barrett 1998:616). Consequently it is recognized that there are two distinguishable forms of psychological kinds for religious cognition: both folk (or implicit) religious representations and theologically correct (or explicit) forms (Barrett 1999):

a) Folk: Governed by online processing - This is sometimes called folk religious reasoning which constitutes automatic, fast and intuitive inferences that conform to the cognitive optimum;

b) Theologically correct: Supported by offline processing - This is constituted by controlled, reflective inferences that conform to a culturally learned knowledge-base ${ }^{14}$

The concept of an omniscient god (for example) remains quite different to the online percept of how agents are normally represented. Further: "a robust concept of god", Barrett argues, is constituted not just by learning a religious creed, but by the implicit (online) and explicit (offline) reasoning about a supernatural concept working in unison (Barrett 1998:617). Reasoning about supernatural concepts online means that mental representations normally conform

\footnotetext{
${ }^{14}$ See Ilkka Pyysiäinen's “Intuitive and Explicit in Religious Thought." Journal of Cognition and Culture (2004) 4(1):123-150 for conceptual overview.
} 
to the processing constraints of our innate cognitive systems. As the Barrett and Keil experiments show, online mental representations regarding 'god' concepts seems to depart significantly from the 'theologically correct' version of god concepts (Barrett 1999). The capacity for offline reasoning, on the other hand, enables the kind of theological complexity that is explicitly expressed in religions. However, the similarities underlying religious systems can be explained by implicit cognitive constraints. Folk religious representations such as anthropomorphic beings and the living dead can be explained by these default inferences. Barrett argues then that religions are made up of both theologies and cognitively optimal (folk) representations.

One thing to notice about these categories is that folk religious concepts appear to be enabled by 'normal' intuitive inferences, whereas theologically correct beliefs and practices are facilitated by enculturation. Theologically correct beliefs require a variety of cultural resources in order to support their cultural transmission (i.e. such as symbols, myths and rituals). This is notable, as explicit religious concepts are often harder to acquire than, say, folk religious concepts (such as $\mathrm{MCl}$ concepts [see Boyer above]). Thus researchers have recognized that despite the transmission of theologically correct belief structures within the cognitive niche, agents still tend to adopt a "theologically incorrect" position when employing religious inferences (see Slone 2004 for discussion). 
With offline reasoning, human agents possess a unique capacity to 'think ahead' or reason about a variety of potential scenarios independently of their immediate context. Kim Sterelny (2003) has dubbed this capacity decoupled representations. Offline thinking or decoupled representations allows for the cortical control of a broader range of behavioural outputs for agents. Put simply, this is the processing channel for information removed from the 'here and now'. Hence, organisms that possess this capacity aren't constrained stereotyped responses coupled to immediate environmental stimuli (i.e. aren't dependent upon just online, single cue/single response mechanisms). Though intuitive reasoning is governed by online processing, non-intuitive or counterfactual reasoning is governed by offline processes. An explicit religious concept such as an omnipresent god is a counterfactual concept because it breaks with our ontological understanding of agency. The capacity to conceive of a supernatural world is an example of this offline reasoning capacity because it exists outside of an immediate real-world and real-time context.

However, the formulation and transmission of explicit religious concepts, I will argue, is enabled by the evolution of cultural technologies such artefacts and symbol-use. It is one thing to have the 'capacity' to generate religious representations and express theological ideas, but it is another to conceive of these 
representations without the use of external cognitive devices. Consequently, the Barrett and Keil experiments don't detract from wideware model. On the contrary, such evidence supports the notion of wideware intelligence. If the cognitive demands associated with engaging in theologically correct concepts are outside of 'real-time processing demands' then we can conclude that external cognitive tools are a necessary component in the transmission of complex beliefs and practices. And both Barrett and Keil would not deny this fact. It's just that they have yet to explain why agents engage in cognitively demanding religious concepts in the first place. And this is the problem that this thesis has been tackling: if it is indeed easier to process representations which conform to a cognitive optimum, then an explanation for why religions are commonly composed of complex theologies is required. Explicit representations are highly valued and costly to believe in.

Whitehouse offers a similar challenge to the SM. On Whitehouse's account, the socio-political arrangement associated with a religious belief system is structured in a way to condition and regulate patterns of behaviour. Religious belief systems often establish a rigorous membership system comprised of costly rituals and expect their adherents to conform to demanding customs. Further, as Whitehouse argues, theological knowledge often exerts cognitive demands for adherents when learning difficult to remember concepts. Hence a value appears to be assigned to the theologically correct position. For 
Whitehouse then explicit belief concepts seem to be just as motivationally salient as the implicit motivations for religious representations. The counterfactual worlds created by human enterprise are both non-intuitive and motivationally salient.

Indeed what separates the "fairies from the gods" Whitehouse argues is that gods aren't just supernatural beings that conform to the cognitive optimum. Instead explicit representations within religious belief systems are usually highly complex concepts that are also dependent upon a rich understanding of the context into which the 'gods' are embedded (i.e. myths, rituals, alters and temples etc). Thus the difference between the 'fairies' and the 'gods', Whitehouse contends, is that the gods are coupled quite considerably to the cultural milieu into which people are embedded - this runs counter to the SM argument that the gods are reducible to certain universal properties. Fairy concepts maybe cognitively optimal, but the Kabbalah certainly is not. Likewise: ghosts are scary, but not as gruesome as god's wrath. Explicit god representations are collectively valued by cultures to varying degrees of concentration. An ancestor spirit may be venerated quite differently from another deity or god. The value assigned to a particular supernatural representation appears to be differentiated by the culture.

This is perhaps a problem for the cognitive optimum then. Because of the memory demands associated with learning and understanding 
the sacred elements within a cultures - constituted by cognitively difficult theological concepts - agents should predictably prefer socalled "fairy" concepts (the cognitively optimal concept) over god concepts, only because "fairies" are far less complex and less costly to believe in (and subsequently transmit). With that said, it maybe hard to conclude that religion is 'natural,' or that all religious concepts are cognitively easy to process. As Whitehouse has noted, societies invest much more energy and resources (i.e. costs) into their explicit beliefs and practices. The question remains: why are individuals committed to such costs?

\subsubsection{Commitments and Costs}

The capacity to conceive of complex cosmologies and theological beliefs raises another important problem for the cognitive science of religion. Offline reasoning can be useful and certainly plays a significant role in our imaginative ability. We can, as stated, decouple our representational competence from an immediate context and conceive of potential scenarios, which is useful for planning and preparation. This is partly why human agents are so innovative and flexible. As Sterelny (2003) and Peter Godfrey-Smith (1996) have recognized, when decoupled representations track the world accurately they become "fuels for success", especially when socioecological environments can be epistemically polluted by others (or if decision-making is blocked by an inability to navigate a 'translucent' 
environment [Sterelny 2003]). Importantly, within a social context agents can strategically lie and give false information in order to reap the benefits of another's credulity. Thus a false belief about the world can have fatal consequences if acted upon.

A major problem then in the study of religion is to explain why individuals not only entertain supernatural concepts and imagine fictional worlds, but also why they invest a great deal of energy, time and resources into ensuring their transmission (Atran 2002; Bulbulia 2004; Dennett 2006). Religious concepts make the epistemic environment very noisy indeed. Individuals express a deep commitment towards ideas which appear to have no utility. On the contrary, religion reduces fitness levels and increases fitness costs. But also, the belief in something that doesn't exist distorts the accurate tracking of the world. As Chris Knight (1998) states, humans implicitly agree to a 'collusion in deception' by inhabiting a world of collectively agreed upon symbols and concepts (see above Chapter 5; Knight 1998). But while symbolic communication plays a functional role, religious beliefs and practices can result in highly costly motivations. Thus it's puzzling as to why individuals would expend the level of costs they do in order to guarantee the gods are sustained within the cognitive niche (Bulbulia 2008).

Some say that the costs of religion should not be viewed as a puzzle but as a causally relevant feature of religious cognition. 
William Irons $(1996,2001)$ recognizes that the fitness-costs incurred

by religious behaviour are outweighed by the benefits of establishing

low-cost policing for effective cooperation. Similarly Bulbulia (2004)

argues that the cost of religious ritual is linked strongly to the

commitments required to perform them. Commitments to religious

beliefs or actions encourage inter-agent cooperation within religious

groups. Rational agents do not cooperate on this view, but irrational

agents do - especially if they believe that a deity will punish them if

they fail to coordinate their interests with others. Hence it is the

commitment reinforced by the costs of commitment that subsequently

counteracts asymmetric strategies for defection ${ }^{15}$.

The epistemic environment is purposefully noisy on this account.

Defective strategies cannot emerge if self-interested agents are

unable to ascertain the costs and rewards of defection. This is true in

most hostile or socially complex environments. For example, in group

environments where the costs of defection is higher or

computationally intractable (i.e. in hostile environments where the

\footnotetext{
${ }^{15}$ This implies that a game theoretical dynamic like the Prisoner's Dilemma underpins cooperative social exchanges. The Prisoner's Dilemma generally describes a situation in which two captives - let's say their names are Roy and Ted - are being held in separate cells for a crime - say a robbery. The prosecution tells them that they can choose to remain silent or confess. But both Roy and Ted are given the same offer: if one confesses to the robbery and the other remains silent, the person that owns up can walk free while the other gets 10 years. However, if both confess they'll get 5 years each. But if they both remain silent they'll get only 6 months each. Obviously, the ideal outcome would be for both Roy and Ted to stay quiet. Yet there is no guarantee that one won't rat out the other (the sucker's payoff would be staying silent while your partner-in-crime talks), so the optimal solution would be to confess anyway. The payoff matrix demonstrates that both should rationally 'defect' against one another. Thus 'cooperation' (i.e. both remaining silent) is an irrational action according to the Prisoner's Dilemma. In biology the Prisoner's Dilemma is used to show that rational decision-making does not necessarily entail cooperation or pro-social behaviour between agents. In a Darwinian sense, it offers a puzzle as to how and why animals do indeed evolve cooperative tendencies. When there is competition, and relative fitness is increased by 'selfish' strategies, agents shouldn't engage in cooperative social exchanges because it isn't in their best interests to do so. Asymmetric strategies are those strategies in social exchanges where interests do not meet (i.e. defective/non-cooperative behaviour).
} 
threat of predation requires group solidarity for defense), the better option is to anchor one's "fate to collective outcomes" in order to avoid the potential risks of non-cooperation or defection (Bulbulia 2007; Dunbar 1996; Skyrms 1996). Thus, polluted epistemic environments can become fertile ground for establishing cooperation. Indeed credulity surfaces ostensibly as a prudent solution to individual risk.

By ensuring the establishment of trustworthy relationships within the community, via costly religious beliefs, the actual costs of policing cooperative tendencies are lowered (Irons 1996, 2001; Sosis 2003; Alcorta \& Sosis 2005). As opposed to more secular policing methods (which may require establishing a strong legal institutional base further absorbing far greater resources), the establishment of an illusionary belief in an omniscient, all-powerful deity reduces the costs of coordination and punishment (Bering and Johnson 2005; Shariff and Norenzayan 2007). The costs of religion generate establishes social cohesion: it culls individualistic behaviour and ensures the stability of cooperation. Self-regulation thus ensures a more stable cooperative milieu, but it also helps to reduce the calculative burden in agency-prediction (see Bulbulia 2007, 2008). The reinforcement of a "shared sense of reality" via symbolically defined ritual communication makes the environment more transparent. 
The claim for religious activities enabling social cohesion seems to be supported by the ethnographic and sociological literature. For instance, an understanding of 'selfhood' especially in small-scale communities is not often constituted by a distinct understanding of identity operating within a world of other individuals and external signs. On the contrary, individuals are merged into a social reality, 'a merger facilitated through symbols... which encapsulate and express the shared subjective experiences of society's members' (Balfe 1985; See her discussion on Durkheim: 240-241). Individuals develop social identities within the milieu into which they are raised, but they also develop a symbolic understanding of themselves and others. Agents recognize how a society is organized based on the interwoven network of symbolically markings, socio-political hierarchies and institutions. A symbolically marked social world makes it easier to track especially if individuals have been habituated by their cultural environments. The ritual communication of these symbolically marked conventions can signal unambiguous signs regarding the social structure within a niche.

Consequently, the psychological states and experiences of individuals within the social structure can be regulated via their implicit motivation to communicate within their symbolic world. Symbols and social rituals are underpinned by certain meanings and emotions. There are certainly social causes for feelings of guilt and regret - i.e. the breaking of certain norms or customs. But further, 
pro-social bonds between kin and non-kin members are constituted by the social structure and the obligations therein. Sexual norms and conventions are a good example of this:

"Families and kin groups cannot organize sexuality for themselves; the partners and patterns they require are usually rooted in wider communities, where lively traditions of sexual prescription-courting behaviour, ritual prohibitions, sexual socialization and the like are played out" (Ross and Rapp 1981).

Cross-culturally we see the social recognition of sexual maturity through initiation ceremonies aimed at adolescents in a community (See Schlegel et al 1980). In societies where it is crucial that adolescent boys for instance acquire an acute understanding of their role within a sexual division of labor there is prevalence of initiation rituals such as circumcision utilized in defining the sexual role of a male during his transition to adulthood (see Young 1980). In societies where male solidarity is critical to the management of cooperation within a division of labor, young males are typically marked for symbolic purposes. Yet it is important that a young male not only be recognized publicly for his sex-role (i.e. the extrinsic value of symbolic demarcation), but also that the adolescent male recognize an implicit sense of selfhood-as-symbol within a larger codified system of beliefs. Frank Young (1980) better describes this dynamic in his paper "The Function of Male Initiation Ceremonies": 
"Identification requires first that the identifier have sufficient skill in symbolic interaction (usually not acquired until early adolescence) to comprehend the symbolic environment and, second, that he recognize that his society requires him to learn certain specific clusters of social meanings, such as those involved in one's sex role. Strength of identification is determined by the degree to which the identifier co-operates in creating and maintaining the definition of the situation and by the degree of clarity given the social meanings by the group or person generating them" (Young 1962:382).

The male initiate gains an entrenched understanding of his role within a society as a result of these initiation rites. The individual is defined by these cultural norms. Commitment is thus established within a collective via these socially defined norms and roles. In contrast to what the SM researchers have suggested, religiosity may indeed be an adaptive trait at the group level for supporting social cohesion and not merely a cognitive by-product of other adaptive features (Bulbulia 2004; Sosis and Aclorta 2003; Wilson 2004). Indeed religious beliefs and practices act as a social regulating system for groups (Wilson 2002). Individualistic behaviour or behaviour that transgresses group coordination can be culled through the doctrinal modes of conduct.

The individual costs of membership then are outweighed by the benefits received from belonging to a collective. Since trust becomes a critical aspect for forming bonds between individuals commitment is 
established via policing - by others' or by a perceived supernatural entity - or by commitments that are merely implicit via enculturation. Hence the social environment becomes easier to navigate when individuals are conditioned to recognize and operate within the symbolically marked features of a particular context (Sterelny 2006). Social environments are made more transparent if agents can recognize the association between the symbolic and the institution. A symbolically marked social organization offers unambiguous information to those who can read these signs. Consequently, agents can better track their social world.

\subsubsection{Motivation and Context}

In contrast to the signaling tradition, others have argued that religious creeds pollute the epistemic environment far too effectively, whereby cultural practices become incompatible with physical environment constraints - thus leading to maladaptive trends (Boyd and Richerson 1985; Dawkins 1976; Diamond 2005). There is no simple answer to this problem, only because religions do not function uniformly across all cultures and societies (Richerson \& Newson 2008). Indeed religions often appear to develop maladaptive outcomes in circumstances whereby certain cultural practices that were adaptive - such as costly displays of commitment for recognizing membership in a group - can begin a runaway selection 
process akin to sexual selection leading to "exaggerated" trends (Richerson \& Boyd 1989; Richerson \& Newson 2008:77).

Thus if the perceived rewards of a certain cultural practice on a micro-level (i.e. within short-term goals) are seemingly outweighed by the costs, then this can generate disastrous long-term consequences at the macro-level (i.e. at the group level overtime). Groups may engage in costly practices which potentially lead to a reduced level of fitness overall (e.g. in the case of female circumcision or foot-binding customs [Sterelny 2008]; or the Easter Island construction of moai statues which evidently led to their socio-ecological demise). So the reinforcement of a collective fiction as a reliable context is both helpful for establishing group-level coordination, while harmful as a short-range and potentially maladaptive strategy.

Despite these concerns, costly religious practices are still perpetuated in most, if not all cultures. So in light of the costs, why has religion prevailed so? This thesis does not wish to entertain a functionalist explanation for human religiosity within an evolutionary context. However, I do think that it is necessary to identify the informational constraints that govern the expression of costly practices. Certainly it is true that epistemic environments - constituted by religious 'fictions' - could be construed as maladaptive (fictions aren't 'fuels for success'). However these environments, though epistemically "noisy" are nevertheless not maximally costly. As 
Bulbulia (2007) has noted, religious information flow rarely impinges nor pollutes - more practically relevant perceptual states and motivations. Thus religious inferences appear to be informationally encapsulated from our more adaptive, common-sense inferences.

Hence, individuals may believe in falsehoods such as "the gods will provide", yet this will not generate runaway maladaptive trends e.g. a false expectation that the gods will indeed provide the material resources required for survival, such as food (Bulbulia 2006). Indeed a prayer before hunting may bring about a belief for favorable results, but the hunters still proceed in their expedition. It appears to Bulbulia (2007) then that the capacity to engage in "counterfactual" worlds is subject to scope syntactic structures within our cognitive architecture (See also Cosmides and Tooby 2000). These dedicated scope operators thus regulate and manage religious information flow in order to control the "inferential hemorrhaging" of religious representations into other perceptual categories that register the world accurately.

Religious belief and behaviour must be context-dependent motivationally. One cannot act on their beliefs in causally irrelevant domains (e.g. one cannot revert to prayer to kill a pig -blades do a better job; though one may pray to the gods for a successful hunt). Some religious representations appear to be coupled to pro-social domains whereby moralistic norms are prescribed to certain types of 
behaviour. A religious inference appears to generate a specific type of content and motivation in these domains (e.g. be charitable or you will be punished in the afterlife). However, these motivations are nevertheless integrated into a specific cognitive system and become causally relevant for the mediation between agents and specific problem-solving tasks (i.e. such as moralistic problem-solving). Moralistic behaviour within groups enables social cohesion and the motivation for this type of pro-social behaviour is context-dependent. Trading with your enemies maybe a bad strategy; however, social exchanges between members of your own faith is beneficial especially if reciprocity is enforced by the gods.

Thus the motivational salience of religious representations are, to a large extent, coupled to certain contexts. If it were the case that religions were just a simple fiction (i.e. just a belief in imaginary beings), then it wouldn't explain why people endow these fictions with a moralistic content. It appears instead, that the tendency to conceive of a moralizing god remains a highly integrated phenomena comprised from social reasoning and counterfactual properties (i.e. those 'fictions' will punish if you copulate with your neighbor's wife [Johnson 2005]). Religious behaviour is constituted by a crossnetworking of cognitive domains. So, it appears as if humans are well-suited to engage in moralistic behaviour coupled to a religious context. 
The theory of a cognitive optimum fails to explain why religious reasoning remains context-dependent (i.e. couple to moralistic behaviour, for instance). Agents tend to employ a great deal of emotional/subjective confidence to some domains, but not others. For instance agents tend to be motivated to act upon emotionally salient (and potentially costly) behaviours in domains that require a degree of subjective confidence to be 'religious'. However, this subjective confidence does not impinge upon other behavioural domains where it would be irrelevant to behave religiously. It could be assumed then that religious representations are evoked in certain contexts but not in others.

This maybe a potentially difficult problem for the SM to reconcile: because while the SM recognizes that there is no "natural home for religion" in the mind, Bulbulia argues that the evidence suggests that there is. Hence the problems and pressures that arise from being embedded in a complex social world - problems such cooperation and trust amongst conspecifics - can be solved by a dedicated cognitive architecture enabling what may be deemed "religious" behaviour (Schloss 2008). A cognitive system that integrates both a belief in the supernatural and a tendency for moralizing behaviour though costly - can be a useful psychological mechanism for trust and cooperation. A commitment to the gods can generate pro-social commitments also 


\subsection{The Religious Cognitive Niche}

An alternative may be to suggest that these behavioural traits aren't governed by an inbuilt cognitive system at all. Within a wideware mind model our perceptual states and behavioural outputs are additionally "anchored" to the material cognitive properties of culture (Mithen 1996a). External media or external cognitive tools can additionally be utilized to manipulate certain perceptual inferences not otherwise availed to our internal cognitive architecture. Conceptual reasoning can be additionally simulated with the aid of certain sociotechnological resources. For example, the concept of the numerical digit 0 (zero), as a place value, was not utilized consistently until the pervasive use of the Hindu-Arabic numeral system (Cajori 1929). Yet the concept's existence was dependent upon the existence of the symbol itself. "Zero" doesn't embody a substantive reality outside of the symbol itself.

This is true of religious cognition as well: for the conceptual boundaries of thought available can be widened with the use of cognitive devices (such as symbols, rituals, myths, artefacts etc). It may be true that there exists internal - and subsequently universal cognitive constraints governing the expression of religious belief, yet what we also find cross-culturally is the use of material artefacts utilized within a religious context also. A concept of what is "sacred" say - and subsequently what is not - is frequently coordinated by the 
placement and collective value of certain external properties within the cognitive niche. Agents organize their world for the transmission of information via the use of material culture. How we represent the world then is at least partially determined by the socio-technological resources available to us.

Bulbulia elsewhere has supported this claim, that socio-cultural practices involve the active modification of the environment to include religious cues: "Agents produce experiential support to back religious commitment. They do so by manipulating their environments in specific ways" (Bulbulia 2008). On this view, agents construct and pass-on artefacts, rituals, and knowledge specific to religious behaviour. For instance, ritualized patterns of behaviour, such as prayer or meditation, increase the likelihood of experiencing altered states. Cultures and societies store and transmit information relevant to altering mental states and regulating the neuro-chemical composition of the body (Newberg and D'Aquili 2000). Recent studies have shown that prayer stimulates the dopaminergic reward system in the brain (Schjødt et al 2008). We can view religious beliefs and activities then as properties individuals desire to be in their cognitive niche.

Societies modify their learning environments to not only support the transmission of religious belief systems and rituals; they also develop a cosmological (or mythic) understanding of themselves and 
their world through the use of socio-technological properties. Thus the offline reasoning of explicit religious concepts will almost certainly depend upon the complex array of distributed knowledge. Indeed Whitehouse recognizes that the offline theological reasoning more than likely requires the scaffolded support of non-biological properties for their expression. The cognitive constraints for modeling these difficult-to-learn concepts are indeed necessitated by material culture.

Material culture is not just a "painted spandrel" as Matthew Day (2004b) calls it, or just epiphenomena generated via online reasoning about the gods. Instead material culture more than likely acts as a "flying buttress" for scaffolding the computational limits of the basic brain when reasoning about the gods. Day argues that studies such as Barrett and Keil's (see above [1996]) have been utilized to defend a position for nativism, undervalued the material culture as being cognitively relevant. Thus the SM views the "ornaments" of material culture as less relevant to an understanding of how religious concepts are represented in the mind. Indeed the focus of examination as a result should be the hardware constraints which govern the processing of this information. Yet Day (2004) argues that the cognitive properties of material culture are a property of the mind.

Hence, Day (2004) adopts an extended mind view of religious cognition, focusing primarily on Andy Clark's model of embedded or extended cognition (see above). Day like Clark suggests that external 
scaffolded culture can be both "context-fixing" and aid as powerful "mind-tools" that "guide individual and collaborative thought" (Day 2004:114 [see Clark 1996]). Indeed Clark has noted that moral reasoning is unachievable without the socio-technological resources of lexical ability utilized in order to co-ordinate activities with others. Clark's concern with the cognitivist view is that it tends to view moral reasoning as the product of individual cognitive processes (i.e. we are capable of being "moral" in virtue of human primate cognitive traits).

In addition, Clark points out that moral reasoning is often a collaborative process involving collective methods of reasoning. Cognitive tools, such as language, enable individuals to establish a moral context into which they can interface. Participating in a cooperative problem-solving activity such as moral reasoning requires a special kind of "knowing" Clark says (Clark 1996:124-125). It not only requires that individuals have a conception of other agent's and their perspective via language, but also that agents utilize lexical cues to shape the content of a moral discourse in order to establish highly cooperative activities with others.

Likewise, Day suggests that material culture enables the contentfixing of religious cognitive processes. In other words agents employ material artefacts to organize and arrange religious thinking. The 'content' of a religious representation is 'fixed' to a material cue (i.e. artefact). How agents represent their world - religious or otherwise - 
Day says is not solely the product of internal cognitive constraints, but are additionally determined by external representations. The temple or alter, for example, embodies a substantive reality in the minds of religious agents, because it exists as a symbolic structure within a culture. The organization and coordination of material artefacts as mental cues function as mental scaffolding for religious thinking.

Day supports Clark's view that cultural practices and devices help us to think and problem-solve. Day suggests then that external cognitive scaffolding aids in the offline reasoning about religious concepts and supports conceptual problem-solving. Hence it “...could put individual human agents in a better position to better utilize their basic perceptual, motor, and social cognitive skills" in religious and sacred domains (Day 2004:117). Day proposes that our folk religious sense can be dramatically augmented and shaped by the cognitive niche. Similarly, to Kim Sterelny (2003) and Michael Tomasello (1999), Day says that basic perceptual modules can be "ratcheted up" to greater precision, Day suggests that external scaffolding can open-up the conceptual boundaries of religious cognition making 'supernatural' thinking more manageable (Sterelny 2003:223; see also Mithen [1996a]).

Day has made this point clear with a comparison between mathematical knowledge and religious knowledge (Day 2005). He argues religious concepts are similar to mathematical concepts 
because they greatly depend upon the development of a symbolic culture. As a result, Day contends that religion is less like a natural language-use capacity and more like the ability to learn complex mathematical models via the use of symbols. Thus, learning the concept of $\pi$ is comparable to learning the concept of Aum, only because of complexity involved in grasping these concepts. Communicating counterintuitive concepts is difficult precisely because the run counter to our intuitive inferences. Religious concepts, like mathematical concepts are counter-perceptive and are necessitate by culturally evolved practices and invention. While our intuitive reasoning regarding basic supernatural concepts maybe constrained by the cognitive optimum (as suggested by the SM), external culture makes it easier to establish collaborative belief systems supported by theological knowledge.

Similarly the SM doesn't often credit the learning of rituals and practices associated with the sacred. Not only are individuals taught a variety of techniques in order to achieve religious states, these techniques are taught and viewed as a component of the religious belief system itself. Explicit beliefs and practices then have a significant role to play in the construction of the religious cognitive niche. These cannot be reduced to just the implicit motivations that underpin cognitively difficult theologies. Instead individuals and societies additionally seek-out explicit religious concepts and ritual techniques for achieving certain mental states. Religious belief 
systems employ elaborate bodily techniques in order to achieve the variety physiological states associated with their commitment to their system.

A prominent feature of religious belief systems is the active manipulation of body for experiential purposes. Subsequently, the experiences derived from experiential rituals via the manipulation of the body are interpreted within a particular symbolic world. So-called mystical visions, meditative and trance states are usually comprised of visions typical to the individual's symbolically structured system of beliefs (e.g. Christian mystics see Christian visions, not Krishnic ones). Yet despite these subjective symbolic explanations for the experience, the experience itself has a very "real" physiological, sensory-nervous correlates (Newberg and D'Aquili 2000: 251-267) ${ }^{16}$.

However there are epistemic correlates to ritual behaviour also (Krippner 2000). Mircea Eliade (1972/1951) often referred to the "sacred technologies" of ritual (See Krippner 2000:93-118 for discussion). This is enabled by the culturally learned techniques of self-regulating psychological functions usually referred to as

\footnotetext{
${ }^{16}$ For instance Newberg and D' Aquili (Newberg and D'Aquili 2000: 251-267) have examined commonalities in phenomenological and observable physiological explanations associated with religious or mystical experiences. Religious ritual and its effects are invariably described within two dimensions (1) 'intermittent emotional discharges' and (2) 'varying degrees of unitary experience'. Yet the subjective phenomena itself has observable neurophysiological correlates. Individuals or groups engaging in particular form of ritualized behaviour are able to stimulate regions of brain associated with emotion, spatial and temporal awareness. Yet the symbolic structures associated with a religious belief system are often recruited in the conceptual understanding of the experience itself. Thus when a physiological change is initiated by the cognitive inputs associated with ritual (i.e. bodily postures, ceremonial movements, meditation and/or prayer etc), interpretations are arbitrarily communicated or phenomenologically interpreted within a particular contextual frame of reference (e.g. Christian Mystics usually experience Christic visions, not Krishnic ones).
} 
religious/mystical experiences. Customs and ritual are learned and transmitted within a symbolic-cultural niche. Ritualized forms of bodily postures and movements for instance are invariably associated with a culture's symbolic milieu and like other forms of epistemic capital they are usually learned and passed-on from generation to generation. However these learned rituals or techniques further stimulate physiological/psychological states, thus regulating a sensory-somatic experience usually defined as "religious". This interaction comprises the "fuzzy area" where biology and culture meet (Glucklich 2001; See Fuller 2007).

\subsubsection{Cognitive Archaeology and the Religious Niche}

Can these claims for an extended mind hypothesis be convincingly supported by physical facts? Has culturally evolved technologies enabled the evolution of the religious mind? Archaeologist Steven Mithen (1996a) seems to think that there is strong evidence for a coevolution of human intelligence and cultural technologies. Mithen argues that an extended mind hypothesis can provide a robust explanation for the cultural evolution of prehistoric societies and subsequently our cognitive expertise. Mithen suggests that the tools utilized by modern Homo Sapiens functioned not only as utilitarian devices, but also as cognitive technologies in very unique ways. The human mind, Mithen argues, is far more flexible than is assumed by Evolutionary Psychology. In addition, Mithen argues that the human 
cognitive processes are integrated and highly confluent with our cultural environments. In other words, we not only employ tools for subsistence (e.g. resource extraction), but we also employ tools for thinking.

However, for Mithen, establishing his own critique of the modularity thesis endorsed by EP seems to be a crucial aspect to model. Thus while Mithen is sympathetic to the view that human minds are comprised of content-rich cognitive domains (the thesis supported by EP), he argues that these cognitive mechanisms may not be as encapsulated, as is often assumed. Nevertheless Mithen argues that human behaviour appears to be determined by a range of cognitive constraints. More particularly Mithen is agreement with EP regarding human intelligence and some of the inborn competences associated with naïve of folk-domain intelligences (i.e. folk psychology, folk physics, folk biology [see above in Chapter 2]). Yet Mithen splits these innate cognitive competences into three categories which he calls Natural History Intelligence, Technical Intelligence and Social Intelligence:

Natural History Intelligence (likely associated with EP's conception of folk biology): a template that enables agents to respond to certain environmental stimuli connected with plants, animals and other biological categories. Humans engage in their natural physical environment competently 
because they possess an innate understanding of some of its properties;

Technical Intelligence (likely associated with EP's conception of folk physics): a template that enables agents to respond to certain environmental stimuli connected with physical dynamics (such as an awareness of length, height, gravity, density etc). This enables agents to be 'novice engineers' if you will, particularly within tool-making domains;

Social Intelligence (likely associated with EP's conception of folk psychology): a template that enables agents to respond to certain environmental cues connected with social exchanges, agent mentality (such as inferences regarding the belief desire states of others) and pro-social behaviour such as cooperation.

If anything Mithen suggests humans should be proficient and flexible in calculating the demands faced by physical environment constraints and social living. However, Mithen is unconvinced that the content-rich systems endorsed by EP are as functionally independent as they assume. On the contrary, Mithen observes that humans are unique in their capacity to cross-network these content-rich domains 
thus enabling the creative processes and properties associated with human primate intelligence within the cognitive niche ${ }^{17}$.

Mithen has illustrated this class of mental flexibility - which he calls "cognitive fluidity" - in a comparative example between human and Neanderthal intelligence (See Mithen 1996a:134-149). He points out that the archaeological record reveals a notable difference in tool innovation between both Neanderthals and Homo Sapiens. The evidence suggests that Neanderthal technology was characterized by a conservative lack of innovation in tool manufacture. Contrastingly human tools exhibited a wider and more inventive use of the local natural resources and materials for tool manufacture (including bone and antler). Yet despite neighbouring the same geographic region with Homo Sapiens - with access to similar resources - Neanderthal tools remained relatively simple and static for a longer period. Mithen suspects Neanderthals possessed a reasonably in-flexible intelligence in comparison to humans.

Mithen argues that Neanderthal intelligence was probably constituted by strictly encapsulated intelligence domains. On Mithen's view their technical intelligence, social intelligence, and natural history intelligence were uniformly specialized and operated independently of one another. Whereas human intelligence he argues arises from an evolved integration of these encapsulated domains.

\footnotetext{
${ }^{17}$ Hence he is somewhere between the MMH and Karmiloff-Smith's (1992) anti-modularity thesis. See
} Mithen 1996:60-61 for short overview. 
With the advent of what Mithen calls 'cognitive fluidity' in Homo Sapiens content-rich systems which had previously been specialized at responding to information specific to those fields of intelligence, gradually became accessible to one another.

Mithen's view of cognitive fluidity has been influenced quite significantly by Annette Karmiloff-Smith's (1992) notion of Representational Redecoration (see above). Indeed Mithen's approach to human cognition is almost certainly similar to Clark's wideware model. On Mithen's view Homo Sapiens intelligence is unique in that information stores in one cognitive domain can be shifted to another domain and applied creatively for or novel problem solving. For Mithen, because information can be cross-networked across multiple domains, humans possess the capacity to apply the information about a certain property, to another set of problem solving tasks. Thus a tool for digging tubers from the ground can also be employed as a tool for scraping or carving. And more unusually, a tool can be used a prestige device for its bearer within a social domain. The possessor of a handy tool can also find value for attracting status.

It is our understanding of the causal properties of the tool decoupled from its actual usage that drives technological innovation. According to Mithen human creativity and depends greatly on our capacity interface cognitive domains (both externally and internally). 
This merge Mithen argues further generated a wider range of cognitive activity that allowed for greater innovation in correspondence with material culture. Hence Mithen argues that the crucial difference between Neanderthal and human intelligence is defined by our capacity to operate effectively with contingent informational resources in a wider range of environments.

However Mithen's is not an argument against domain-specifity as such. His skepticism with strictly encapsulated domains is derived from the observation of "non-specialized" thinking within human primates, whereas the Swiss-army knife analogy for domain-specifity seems to imply narrow specialization (i.e. specialized "tools" for specialized tasks). For instance, he argues that individuals tend to think of the natural world in technical terms as outlined above with the Neanderthal example (Mithen 1996a:49). Additionally individuals tend to imbue their environment with a humanly significant purpose, for which Mithen says is an indication of social intelligence and natural world intelligence merging.

As stated, humans throughout history have tended to imbue their cultural artefacts with a social currency leading to possessions being valued as sexual ornaments or items of prestige. Subsequently Mithen's concern over the EP project is that domain specifity tends to entail specialization in specific task-domains. Thus on the EP view we should only 'act social' in social domains; or be 'tool-makers' in 
technological domains; and reason about biological categories as natural history intelligence - yet operate within these task domains well as domain-specific "geniuses". But as Mithen points out, human beings conceptualize their worlds in varying, non-specialized ways. So while Mithen doesn't deny that we possess content-rich domains specific to certain tasks, these content-rich processors do not determine specialized behavioural traits. Indeed they are merely a backdrop enabling the integration of powerful representations across a variety of domains for greater flexibility.

Mithen is concerned with the effect that material items (symbols, tools etc) would have had on the environment and the agents that inhabited them. Put simply, the cultural evolution of materiality constitutes a merging between brains and environment. Again Mithen differs to the orthodox view of EP, as it is suggested that human cognitive fluidity facilitates the cross-networking of external and internal cognitive domains. Homo Sapiens has managed to integrate both the content-rich domains of their cognitive architecture with the external properties of their material world.

Mithen argues that this dynamic has facilitated a co-evolutionary process between minds and environments thus producing a human cognitive niche. Thus a spear or pot is not just an extrinsic property in agents' world for utilitarian purposes only (i.e. a hunting implement or container implies a function to the archaeologist). But such devices 
contain epistemic information, or more simply a blueprint for the construction for further spears or pots. In short Mithen argues that material culture would have served a purpose additionally as an external memory device for individuals and societies (Mithen 1996a). Agents do not depend upon the informational load that comes from memorizing how to manufacture these artefacts. The item in the world - coupled with Homo Sapiens content-rich multiple intelligences - establishes a powerful tool for learning and the transmission of valuable knowledge.

Mithen's model for the co-evolution of human cognition remains compelling for it implications alone. Mithen is certain that the intensification of material culture is correlated with Homo Sapiens cognitive expertise. More specifically material culture in the form of cognitive technology (i.e. symbols, tools etc) enabled the capacity to integrate intelligence domains that were once quite inaccessible to each other. Thus social worlds adopted properties from the technological world: for instance the use of something functional, such as a pot as a container, might be deemed a prestige item in the social worlds of agents. Thus when stylistic properties such as pot motifs and designs began to appear on tools, this should additionally indicate to archaeologists that utilitarian items were becoming integrated into a more abstract (non-utilitarian) contexts. 
Archaeologists should then infer that individuals were becoming increasingly aware that inert objects could be endowed with meaning and significance within a social context. An explanation for this reorganization of cognitive-knowledge domains is still unclear according to Mithen. Safe to say however that is was not the result of any major genetic change in human cognitive evolution. Thus a shift from a strict domain-specific intelligence to cognitively fluid intelligence cannot be the result of any momentous physiological change in human evolution. Mithen does say however that a probable explanation for this change in intelligence was significantly influenced by the appearance of material culture itself. Once external material cultural items began to occupy the human cognitive niche, their existence influenced the capacity to conceptualize new and innovative ways of thinking and behaving.

For example, the appearance of certain symbols coupled with human linguistic competence produced the capacity think more abstractly about certain cognitive properties. Thus the presence of symbolic material artefacts would have coincided with the capacity to store and transmit information in a non-biological format (similarly to Donald's view of external symbolic storage [see above]). Hence a coevolutionary process took hold, initiating a shift in the way individuals conceptualized their world. For example, Upper-Paleolithic cave painting in particular images such as the depiction the Bison with human legs found in the Chauvet Cave in France should indicate, 
according to Mithen, an integration between thinking about biological categories (i.e. the non-human animal world) and social intelligence (i.e. the human psychological world), resulting in the capacity symbolize metaphorical associations such as anthropomorphic beings (Mithen 1996a:186-190; Srejovic' 1969).

So for Mithen the external symbolic items of material culture have consequently shaped how the internal dynamics of human thinking is directed and vice versa. By storing an image within the cognitive niche, agents were able to better process difficult concepts such as anthropomorphic symbols. The computational limits of our internal cognitive machinery are thus better supported by material symbols. Thus from what we know about human cognition - plus - how these processes interact with material culture, Mithen hopes that cognitive archaeologists will be better equipped to understand how material culture - and particularly symbolic culture - would have "anchored" the development abstract - or non-intuitive - concepts (Mithen 1996a; 2000).

\subsubsection{The Emergence of Symbolic-Religious Behaviour:}

Archaeologists are often careful however to not separate fact from inference. For instance, a guiding rule could be to assume a noncollapsible distinction between the functional material items of a society's economy and the non-functional items of a culture's beliefs 
and practices (see discussion on Hawkes' ladder [Hawkes 1954;

Robb 1998]; Jeffares 2002). Hence the 'visible' facts regarding the function of particular tool (e.g. a hand-axe) is often relatively easier to infer over the 'invisible' facts (e.g. the belief of the bearer). Put simply, the "hard realities" of economy and environment are supposedly much more accessible to the archaeologist than that of symbolic interpretations (Robb 1998:330). 'Meaning' is potentially an inaccessible reality within archaeology, whereas functionality and economy can be measured.

Because of this problem of interpretation, the inferences regarding the meaning of symbols from long extinct cultures have been openly criticized by archaeology. Inferences regarding religious beliefs and practices, for instance, can be subjective and open to a variety of interpretations. Some have criticized this separation between function over meaning however. Archaeologist John Robb (1998), for instance, sees this common prejudice within archaeology as a fundamental bias which eliminates the potential for a more interdisciplinary approach and interpretation:

"The archaeological world is a cultural world, and by dividing into a priori categories of material and symbolic, we deny the degree to which things like economy are fundamentally cultural things like ideas are embodied in cultural practices" (Robb 1998:331). 
Yet archaeologists may argue that these concepts or symbolic structures are just representatives of a social reality that exists prior to their appearance in a cultural setting. Concepts of value and exchange reflect the economic nature of human beings: an extension of a fundamental reality. Hence, symbols merely serve as "tokens" or "instruments of communication" (Robb 1998:332). Thus a set of symbols may be representative of an individual or group's power and prestige. So in the case of a particular type of adornment or burial, an archaeologist can assume the social standing of the individual. On this view the material artefacts of the examined culture has been used to symbolize - or represent - the hierarchical structure inherent within the society. Yet there is a problem with this assumption. One cannot assume the existence of a social context prior to the symbolic concept. As Robb (1998) points out:

"Its most problematic assumption is simply that artefacts, actions, and social relations have a meaning or existence logically prior to their translation into symbols, which serve primarily to represent this perceptual reality" (Robb 1998:333).

Archaeologists and ethnographers should not assume a presymbolic reality underpinning the cultural production of concepts and representations within a human social context. Symbols aren't merely "projections of antecedent concepts, but in its substantive reality [they are] constitutive of the concept" (Renfrew 2001:130). The methodological individualist framework that has pervaded 
archaeology presupposes symbols, representations and - inevitably the complexity of culture, as just a by-product of behavioural traits fundamental to human social/economic living. Yet the context in which symbolic structures are produced requires that humans be in the business of constructing a contextual environment scaffolded by material culture. Consequently, the material culture that is derived from individuals engaging with these symbolic representations reproduces the social practices which constitute a particular worldview relative to the cultural and symbolic environment (Barrett 2001:152).

Thus 'meaning' doesn't exist independently of a cultural and symbolic structure. On this view then symbolic structures are highly embodied, requiring a substantive reality to be present prior to the concept (Renfrew 2001; 2008). As archaeologist Colin Renfrew (2001) points out, though these cultural structures certainly serve a function, the symbol itself shouldn't be viewed as an inactive referent to an already existing concept. Nor should a 'context' be presumed prior to the reality of the symbol. Instead, for Renfrew, social realities are constituted by symbolic structures: the symbol precedes the concept (Renfrew 2001). The symbol of 'wealth' for instance in a particular society is coupled to a substantive reality: someone with lots of stuff. Wealth is enabled by a substantive reality of individuals who are symbolized as wealthy in virtue of their private capital. Likewise, with religious belief we cannot assume the religious nature 
of individuals prior to its expression in a culture. The symbolic structures comprising religious behaviour (e.g. alters, rituals, beliefs etc), constitute the social reality of religion itself.

Renfrew (2008) also notes that societies consign value to objects, materials and locales independently of utilitarian value. He gives the example of the intrinsic value of gold. What is found in the archaeological record, however, is a complete lack of value assigned to this material before 1000 years ago. Renfrew notes an obvious fact, that: "The intrinsic value of gold, is of course, culturally ascribed" (Renfrew 2008:2044). But what is of importance here, Renfrew asserts, is the "very notion of value itself" (2008:2044). In religions, cultures the attribute value to 'sacred' material items and our ability to coordinate our niche into a system of representational properties with intrinsic value is apparent. The intrinsic value 'sacred' materials are not only endowed with meaning, but they are also motivationally salient to adherents.

Mithen (1996a; 1996b) takes a similar approach to Renfrew's with regard to the transmission of religious beliefs. Material artefacts and symbols play "a role similar to that of language in terms of creating networks of minds, disembodying minds, and exponentially increasing the range of conceptual spaces available for exploration and the manner in which this could be undertaken" (Mithen 1996a:181). Mithen suggests then that religious-symbolic structures 
are essentially 'consubstantial' with human cognitive architecture (see also Malafouris 2004). Thus, the existence of religious symbolic structures in a cultural milieu essentially "anchors" the perceptual categories of an agent's social reality in regards to religious belief producing concepts that would not normally be possible without material symbols:

"Hence, rather than having a shared biologically based anchor in our brains that allows for the persistence and transmission of religious ideas, we have adopted material anchors in the form of either abstract or naturalistic depictions. The mind has simply been extended into the material world to extend the range of concepts that it can think about - to explore new conceptual spaces" (Mithen 2001:110).

Thus while Mithen maintains that Boyer's picture of religion is compatible with his picture of the mind being cognitively fluid, Mithen argues that counterintuitive representations are in fact dependent on our capacity for cognitive fluidity (see above), without which human cognition could not deploy such powerful representations. Since cognitive fluidity allows individuals to "[bring] together knowledge and ideas from different cognitive domains" (Mithen 1996b:101)], we can engage in the counterintuitive representations that Boyer deems as important to the transmission of religious beliefs. However Mithen holds that the transmission of religious ideas is considerably dependent upon material culture. Hence, the appeal of 
counterintuitive concepts cannot be explained in virtue of internal resources alone. Neither can their transmission. Environments are difference makers, not merely prompts and triggers.

In addition, because our cognitive architecture is not well-suited to the transmission of these ideas - due to their counter-intuitive features - the spread of religious representations requires a robust concentration of religious material artefacts in the world to make them more memorable. Thus religious counterintuitive representations "do not relate to an evolved feature of [our] mental architecture. In an evolutionary context they do not 'fit' into the domain specific cognitive domains, and in a developmental context they do not 'fit' into a domain of intuitive knowledge" (Mithen 1996b:102). So Mithen is saying that counter-intuitive concepts are far too cognitively intensive to process via our cognitive architecture alone. He argues that religious concepts, in particular, require support from material symbols in order for them to be transmitted.

Thus religious belief is not just 'evoked' necessarily, as Boyer suggests, instead Mithen argues that there exists a natural codependence between both our external cognitive domain (stuff in the world) and the internal one (our content-rich and cognitively fluid hardware). The generation of supernatural cognition is dependent on the cultural niche to 'anchor' it as much as it is dependent on cognitive fluidity. Indeed it requires the existence cues present within 
our external environment in the form of symbolic representations (such as rituals and idols etc).

A 'religiously' imbued symbolic marker thus acts as an anchor for the human mind and supports the biological limitations of our basic brain for cultural transmission. For Mithen then this remains an important development for human cognition in the emergence of religious belief. As stated, without the capacity to merge cognitive domains, with the meta-representational abilities associated with cognitive fluidity Homo Religiosis would not have evolved. It is the substantive reality of symbolic culture within the human cognitive niche that would have initiated this trend towards the supernatural. Put simply the internal dynamics of pan-human cognitive architecture is insufficient to facilitate the spread of religious ideas in the wild.

\subsection{Summary and Conclusion}

A problem for the SM is that not all religious representations conform to a cognitive optimum. Thus (a) societies tend to favor a preference for cognitively costly concepts and rituals: hence the theological complexity embodied by many religious beliefs and practices often exert large demands upon their adherents to secure and transmit. Also (b) the SM is at odds to explain the contextdependent nature of religious representations. If religions are constituted by cognitively optimal supernatural concepts then why do 
the gods not just look, but also behave the way they do. For instance, as many observe, religious representations are often evoked within a pro-social or moralizing context. However while some concepts remain coupled to certain culturally relevant qualities (such as an authoritative agent who governs social norms and moral behaviour), there are also those supernatural concepts that are not revered in a similar way i.e. those "fairy" supernatural concepts, which are more benign. Counterintuitive concepts seem to vary in complexity and costs.

Thus one way to think of the theological "distortion" that pollutes an epistemic environment is to conclude that it is of no concern. Despite the elaborate theological systems that are produced within the cognitive niche, cognitive processes are always regulated by the optimum when reasoning online. Thus despite the propensity to remain "theologically correct" within a religious context, agents always employ folk religious inferences regarding the gods.

However this does not explain the 'costs' involved in believing in some supernatural concepts. Offline theologies are distortions par excellence. People exert a great deal of individual and collective energy in order to commit to them. Thus explicit concepts are just as motivationally salient as implicit inferences regarding the gods. This gap between online and offline reasoning about supernatural concepts may need to be closed if the SM is to make any advances 
in explaining the qualitative differences between folk religious concepts and theological correctness. Indeed as Donald proposed (see above), with the advent of a technologically supported theoretic culture societies often function within a shared code of "theologically correct" belief structures (Barrett 1999; Pyysiäinen 2004). This is where Whitehouse's reading on the processes and properties associated with the doctrinal mode is helpful in interpreting how the distribution of knowledge within a society supports religious transmission.

Social and cultural structures, he argues, enable a robust collective knowledge-base and the interpretation of publicly represented symbols. Because the doctrinal mode exploits semantic memory, according Whitehouse, agents learn and are conditioned to respond tacitly to a variety of external symbolic cues. Yet these tacit responses are learnt, not genetically embedded. Indeed, a Catholic cannot remember how they learned the representational qualities associated with the Crucifixion, yet there remains an unspoken commitment to this symbolic reference. Thus the integration of symbols and its cognitive comprehension remains the product of the deliberate and gradual process characterized by indoctrination.

Cognitive archaeology raises some valid points and Renfrew's argument against the more traditional (or processual) archaeology 
should be iterated ${ }^{18}$. Instead of symbols being the derivative of a fundamental reality (i.e. a general law of human behaviour), they view symbolic material culture as cognitive properties in their own right. Mithen and Renfrew support the view then that symbolic material culture constitutes a specific environment that would have not existed without socio-technological artefacts. In particular, a symbolic reality precedes the conceptual reality of a particular culture. Thus specific worldviews and creeds for example would not have existed independently of these mind tools. The symbolic cognitive niche scaffolds the mythic and theoretic culture of a society. Since religions - particularly their theologies - are founded upon a conceptual reality unique to a specific belief system, then we can also conclude that material symbols support modes of transmission within the religious cognitive niche.

The spread of any theological concepts require socio-technological modes of communication (such as language, writing skills, parchment technology, ink and the invention of the printing press to name just a few) to facilitate their spread. The concepts inherent within any specific religious worldview do not appear to be governed by an instinctive disposition towards a particular belief or concept. Nor can it be assumed that the success of such theological concepts can be facilitated by the content of the representations (i.e. their anthropological properties etc). Thus, the prediction that the cognitive

${ }^{18}$ See Lewis Binford, Archaeology as anthropology. In Contemporary Archaeology, ed by M. Leone, pp. 93-101, 1962; Or Bruce Trigger's A History of Archaeological Thought. Cambridge University Press: New York, 1989 for an excellent overview of the development of archaeological theories. 
optimum enables for the transmissive frequency of minimally counterintuitive beliefs falls short of the ethnographic and archeological data. Cognitive archaeology has set-out to highlight this point also: that cultural transmission, in general, is greatly determined by the epistemic properties of material culture. 


\section{Chapter 8: Conclusion}

\subsubsection{Overview:}

The cognitive science of religion is a multi-disciplinary approach to the study of religion and psychology. However at its core the cognitive science of religion emphasizes the importance of empirical research. A scientific approach has subsequently been applied in order to determine testable methods and theories for examining the causes of a particular cultural phenomenon. The concern that researchers of religion had initially was that more traditional approaches offered poor explanations for the psychological factors surrounding human religious behaviour. Traditional anthropological approaches, for instance, are an interpretive enterprise, thus the methods employed often depend upon non-testable processes of examination. The cognitive science of religion has been concerned more notably with: a) how religious representations are produced; b) how these representations are transmitted; and c) how it is that we have the kind of evolved cognitive architecture capable of doing both a) and b).

However, how religious representations are produced have nothing to do with a particular feature of our brain devoted to religious reasoning. On the contrary, common features of the brain devoted to 
doing other tasks may indeed be susceptible to or generate religious ideas. For instance, according to the Standard Model, religion is a byproduct of a variety of other cognitive systems in the brain specialized for doing other tasks (see Chapter 3). The SM is founded upon the notion that our cognitive systems can instinctively recognize certain ontological types such as physical, psychological and biological categories in the world, consequently the SM argues then that religious ideas hijack our perceptual categories because they are 'counterintuitive' to these ontological types. According to this view religious ideas excite our understanding of what is considered 'natural'. In this case, cognitive systems that are functional in one sense, are non-functional or operate in a non-standard fashion within a 'religious' context.

Chapter 4 offered some of the alternatives to the more orthodox perspectives within Evolutionary Psychology. I began with Merlin Donald's argument against Evolutionary Psychology's notion of domain specific systems in the brain. Human intelligence, Donald argues, can only be partly recognized as the result of genetically evolved traits. Evolutionary Psychology, according to Donald, overlooks the integrated features of our brains with our cultural environment. The neurological evidence he says does not demonstrate the existence of specialised sub-computers in the brain, instead the most recent neurological development in Homo Sapiens is the enlargement of the pre-frontal area which is functionally plastic. In 
addition, the neuro-constructivist account for learning development (Quartz \& Sejnowski 1997; Quartz 1999) - in which the structuring of some neural circuitry is shaped by environmental factors - supports the argument for plasticity also.

Donald proposes that it is this combination of brains and cultural environment that has made us smarter. This dynamic he dubs the 'hybrid mind' which he says has emerged due to co-evolutionary processes. It is this cognitive flexibility that remains a unique feature of human beings, not inbuilt specialized or content-rich systems. It is this connection between our 'internal' cognitive domains and our 'external' cultural domains which constitutes a 'scaffolded' intelligence. Indeed we inhabit a cognitive niche comprised of nongenetic resources that support our cognitive expertise. And because human beings are ecological engineers - in that we shape and reshape our environments - we are niche construction agents who inherit and pass on these scaffolded environments to each generation.

I argued that the hybrid mind, niche construction and extended mind models offer compatible theoretical challenges to the EP model; each shared components which I felt suited Andy Clark's notion of a 'wideware' of human cognitive model (Clark 2001). Clark's wideware model illustrates that cognition is an emergent property arising out of an interface between brain, body and environment. Our everyday 
practices involve the use of 'cognitive technologies' such as language, pens, paper and computers. These are not only artefacts in the world, but our interface with them requires the transmission of socio-cultural practices such as reading, writing and the use of welltrained motor-skills. We are nothing without the voluntary control of our bodily movements, just as the printing press is function-less without general literacy. A cultural environment is crucial for this kind of learning and development, just as it was important in the training of Kanzi the chimpanzee to use symbols to communicate. With the appropriate training, in the right environment, Kanzi learned to make associations between real world cues and symbolic representations. Enculturation is vital to our cognitive expertise and a wideware understanding of human cognition provides an appropriate examination of how we interact with our cultural niche.

More importantly to hominine evolution, however, was the development of symbolic culture. A symbolic culture has enabled the capacity to not only pass-on valuable information from one generation to the next, but it also supported the development for learning 'higherorder' representations such as abstract supernatural concepts. Thus in Chapter 5 Merlin Donald's co-evolutionary model was then further elaborated. I focused on Donald's theory for hominine cognitive evolution, which splits the development of hominine intelligence into three major transitional periods in our cultural evolutionary history (mimetic, mythic and technologically supported culture). It is hominine 
activity (or niche construction as I viewed it) that has driven the evolution of cognitive traits. As hominines shaped their environment, so in turn their environment shaped their intelligence (Sterelny 2003). It has been the capacity to engage in collective communication and the distribution of symbolic cognitive tools which has supported the development of representational thinking in our hominine ancestry. This first began with the ability to utilise the body as a public representational device, which led to a spoken language, further leading to the evolution of a 'technologically supported' symbolic material culture. And it is this third stage of development identified by Donald which has been the focus of this thesis.

Because theological complexity within religious beliefs systems is the cause and result of a symbolic culture, the cognitive demands associated with the transmission of these concepts depends greatly upon the development of technologically supported cultural traits (e.g. religious texts are a good example of this). This actively external dynamic in the evolution of human cognition would have allowed for the processing of cognitively difficult beliefs and practices, particularly with the development of complex theological concepts.

Chapter 6 presented some of the problems surrounding the Standard Model's conception of human cognition and religiosity. The Standard Model researchers covered in this thesis were prepared to categorize certain cognitive traits, which they suggested were stable 
features of human cognition, prior to an examination of religiosity. This I argued was a questionable approach, as the cognitive system itself had only been partially explained on the outset. In addition their operational definition of religion was insufficient. As Harvey Whitehouse illustrated in this section, religious belief systems are constituted by much more than universally identifiable features such as agency (i.e. anthropomorphic representations), or minimally counterintuitive concepts. Belief systems are additionally comprised of socio-political arrangements and ritual norms. Thus the focus on Whitehouse was intended to illustrate a growing dissatisfaction with the SM approach. It was important to examine here Whitehouse's critique of the SM and particularly his argument against the cognitive optimum. Whitehouse was skeptical of the so-called universal properties identified by the SM within religions. In other words, Whitehouse is critical of accounts for which innate features of our cognitive architecture regulate particular religious representations and govern the expression religious behaviour.

Whitehouse urges that religiosity is much more context-dependent than is readily assumed by the SM. The religious mind he argues is constituted additionally by socio-political arrangements and the differing modes of ritual specific to the social, material and ecological constraints of a particular culture. Put simply, a religious belief system is adapted to the circumstances of a particular context. I intended to establish correlations between Whitehouse's modes theory and 
distributed cognition models. Here Whitehouse has offered a unique solution to a troubling question within the cognitive science of religion: why do religions look the way they do? Belief systems, Whitehouse argues, are subject to the selection pressures of particular socioecology. I argued that Whitehouse's theory is similar to a distributed cognition model as religious transmission is shaped by external dynamics not just internal cognitive regularities. The distribution and arrangement of socio-technological properties thus support the flow of religious information within cultures.

In response, I argued that this conjecture supports the evidence for a wideware model, because the transmission of explicit or cognitively difficult religious concepts will subsequently depend upon the development of cultural tools (e.g. symbolic artefacts, texts, ritual etc). Further, I also argued that the cognitive science of religion shouldn't underestimate the commitment that adherents have towards these explicit representations. On the contrary, explicit religious concepts, or 'theologically correct' concepts (though dependent upon cultural learning), have been found to be just as motivationally salient as the innate processes which guide implicit religious inferences. The costs that are required for engaging in these beliefs and practices are apparent and suggest that individual commitment is guided by something more than intuitive inferences. Explicit religious representations have more value to religious 
adherents than the cognitively optimal supernatural concepts identified by the SM.

Though religious concepts congest an epistemic environment, a religious cognitive niche also supports cooperation between agents within in groups. Social transparency then is context-dependent only because those individuals who adhere to a particular belief system can indeed make successful social exchanges via commitment. As stated, religious beliefs and practices can be a powerful culturally evolved mechanism for social cohesion. The pro-social aspect of religiosity in part explains why the religious cognitive niche contains representations that are pro-social or moralizing in their scope. The common pro-social aspects associated with religious behaviour (i.e. such as moralistic norms) however don't imply that there are internal cognitive systems specifically coupled to religious inferences. Instead the content of religious representations - whether they are moralizing or not - relates to the capacity to shape cultural properties within their environment in order to influence the behaviour of its inhabitants. In order to examine religious behaviour a more comprehensive model of the religious mind should focus to the intersection between brains and material culture.

While sympathetic to Mithen's view, I observed that the data remains insufficient. Archaeological 'facts' do not exist independently of interpretation. Evidence is critically dependent upon interpretation 
of scarce material evidence. A potential setback then for the theoretical models supported by Mithen's thesis is the need for more archaeological data. But this of course is a problem for archaeological models of interpretation: a shortage of details. Mithen has avoided this problem however by employing facts regarding our cognitive architecture to the interpretation of archaeological finds. This is a crucial step in the right direction, because archaeologists can at least attempt to make stronger inferences regarding the thoughts and beliefs of agents - i.e. those 'invisible facts' - from these long-lost cultures. By deducing some cognitive regularities and how they function in concert with material culture, Mithen is surmising that we can build a better picture of how material culture influenced our cognitive skills and vice versa. Inferences regarding material culture can be turned upon ancestral thinking and human cognition. Subsequently, facts regarding human cognition can be turned upon inferences regarding material culture.

However, because a wideware model implies a correspondence between 'culturally' evolved traits and 'biologically' evolved traits, it is archaeological data that will be able to offer sound inferences regarding the evolution of the human cognitive niche. There is little that can be said on this matter, not only due to the 'lack of data,' but also because archaeologists have yet to agree upon their 
interpretations ${ }^{19}$. Nevertheless, since the cognitive sciences applied to human religiosity and archaeology is still a relatively new emerging field, there is indeed a great deal that we may be able to understand if we can successfully integrate these fields of research. What we should be looking for - in conjunction with perhaps Whitehouse's doctrinal modes theory - is a greater concentration of religious material culture emerging alongside an increased level of social organization. Social complexity should produces cultural complexity and vice versa. Archaeological interpretations should provide a clue as to whether or not this is accurate in the case of religious activities.

\subsubsection{Where is 'religion'?}

The central point of this thesis has been to explain why religious behaviour is coupled tightly to the cultural properties of the human cognitive niche. Explicit representations within religions are often uniquely associated with cultural learning, while theological concepts are highly valued as costly commodities. Yet the Standard Model predicts a greater prevalence of less-costly representations (those representations which conform to the cognitive optimum), while overlooking the challenge of theological complexity. However, religious information is constituted by conceptual knowledge that is, in large part, learned by cultural actors. While the Standard Model allows for the importance of cultural learning, they labor under an

\footnotetext{
${ }^{19}$ See Ben Jeffares' The Scope and Limits of Biological Explanations in Archaeology (2002) for an excellent overview of this debate.
} 
incomplete picture: that the properties of religious representations are founded upon context-independent causal influences. Though environments are important, for the Standard Model it is our innate cognitive systems that govern the expression of religion. However, as we have seen, theological knowledge is often comprised of concepts that would be cognitively difficult to process without use of symbolic artefacts.

Religious agents seek out this kind of engagement also: they regularly interface with computationally heavy concepts by way of religious material culture. This makes theological complexity interesting because it embodies an informational load that makes transmission difficult. The Standard Model on the other hand argues that human cognitive systems are susceptible to supernatural ideas (especially Boyer and McCauley), when it is not obvious that we are somehow predisposed to religious ideas - especially when they are so costly to acquire. Again, religious information is costly to acquire. Yet, human societies and cultures appear to be satisfied with not only absorbing these costs, but seeking them out also. We exert a great deal of effort in the construction of our religious niches.

My central claim has been that complex bodies of theological knowledge require the use of symbolic artefacts for transmission. By this I mean that conceptual reasoning is enabled by symbolic communication (as Colin Renfrew states: 'the symbol precedes the 
concept' [Renfrew 2001:130]). As concepts become more complexin the case of religious representations - material symbols are required in order to support their transmission. This provides a suitable explanation for why religious belief systems are founded upon external media devices, and why the 'sacred' is characteristically represented in societies in the form of collectively valued material symbols.

However, though it is important to recognize the psychological preconditions for human religiosity - those genetically endowed resources - material culture remains a psychological foundation for religious thought. Further, the material basis of thought requires us to rethink the role of evolution in shaping the religious mind. Societies and cultures adjust and adapt to their religious cognitive niche via the transmission of cultural properties which essentially augment conceptual thinking within a religious context. The 'sacred' then should be viewed as the content-fixing of concepts through the use of religious 'mind tools' (see discussion on Matthew Day and Andy Clark in Chapter 7; also Day 2004b; Clark 1996).

Because explicit conceptual reasoning depends on our capacity for offline thinking, external symbolic media remains crucial to the processing of religious information. Moreover that thinking is affected by contextual as well as developmental factors. Indeed cultural artefacts are essential for collaborating religious collectives as they 
allow for the organization and the transmission of the 'sacred'. Thus explicit beliefs represented in the cognitive niche as artefact should not be overlooked. As Matthew Day points out here:

"By treating cognition as something that happens inside the head, we run the risk of habitually overestimating the biological brain's natural computational prowess and underestimating the consequences of non-neural cognitive resources. More devilishly, we may also end up with a skewed portrait of the mind's evolved design." (Matthew Day 2004b:106)

It is indeed these "non-neural cognitive resources" that this thesis has chosen to focus on here. Moreover, I have highlighted research that offers new approaches within the cognitive science of religion outside of the representationalist paradigm. Symbolic artefacts thus should be viewed here then as non-neural cognitive resources that scaffold the biological limitations of our religious thinking. This subsequently warrants an examination of culture as being causally relevant to an understanding of human cognition.

Contrary to McCauley's claim (see Chapter 3), religion is a lot more like science, than it is a 'natural' cognitive disposition. Like science religious belief systems establish causal explanations for the world and subsequently perpetuate a rich lineage of material cultural artefacts to transmit these worldviews. For the religious practitioner there exists a similar informational load comprised of theoretically 
challenging concepts which exist outside the boundaries of our 'native' intelligence. Religion is not for 'dummies', as so implied by McCauley's comparison. On the contrary, religious belief systems are comprised of highly complex systems of knowledge. I have argued that if the cognitive science of religion is to make any headway it should view the study of culture as a level of organization that affects individual behaviour - particularly our religious beliefs and activities. As religious scholar Gabriel Levy states:

"Since the brain develops in the context of non-cognitive physiological and network processes, and concepts and actions in the world depend on non-cognitive physiological and network processes, we can say that these processes are just as important to understanding religion as those processes that take place in individual heads" (Levy 2005:194).

Certainly the research which has attempted to isolate psychological features - outside of the cultural context - as a causal explanation for religiosity has been helpful. But it shouldn't let us inform our definition of 'religion'. As we have seen, human religiosity is not just a belief in anthropomorphized supernatural beings, nor is it solely rituals. Instead religion (whatever it is), appears to be a highly integrated phenomena encompassing these things plus the institutional, political, cosmological and the material properties of a particular cultural. We can isolate these properties, but it would be foolish to consider them purely the result of our neurological 
characteristics. This thesis has argued that 'human religiosity' (whatever it is) extends beyond the cranium. We can argue then that since cognition functions at differing levels of organization (e.g. brains, body and environment), then human religiosity similarly operates at differing, yet compatible, levels of organization within the cognitive niche. A wideware interpretation of religious behaviour means that there is no simple way to view the larger whole from just its distinguishable parts. Instead, we should begin to identify how the parts interact and are coordinated within a 'religious' network. 


\section{Bibliography:}

Alcorta, C. and Sosis, R., (2005) Ritual, Emotion, and Sacred Symbols: The Evolution of Religion as an Adaptive Complex, Human Nature 16:323-359

Atran, S. (1998) Folk biology and the anthropology of science: cognitive universals and cultural particulars. Behavioural and Brain Sciences, 21: 547609.

Atran, S. (2002) In Gods We Trust: The Evolutionary Landscape of Religion, Oxford University Press, New York.

Baldwin, M. J. (1896) A New Factor in Evolution. The American Naturalist, Vol. 30, No. 354, 441-451.

Balfe, J. H. (1985) Sociology and the Sublime. New Literary History, Vol. 16, No. 2, The Sublime and the Beautiful: Reconsiderations. pp. 237-249.

Barkow, J., Cosmides. L. \& Tooby J. (eds.), (1992) The Adapted Mind: Evolutionary Psychology and the Generation of Culture. Oxford: Oxford University Press.

Baron-Cohen, S., Tooby, J. \& Cosmides, L. (1997) Mindblindness: An essay on autism and theory of mind. Bradford.

Baron-Cohen, S., Tager-Flusberg, H. \& Cohen, D. (2000) Understanding other minds: Perspectives from developmental neuroscience. Oxford University press.

Barrett, J. C. (2001). Agency, the duality of structure, and the problem of the archaeological record. In Hodder, I. (ed.), Archaeological Theory Today, Polity Press, Cambridge, pp. 141-164.

Barrett, J. L. (1998) Cognitive constraints on Hindu concepts of the divine. Journal for Scientific Study of Religion 37:608-19.

Barrett, J. L. (1999). Theological correctness: Cognitive constraints and the study of religion. Method and Theory in the Study of Religion, 11, 325-339.

Barrett J. L. (2000) "Exploring the Natural Foundations of Religion," Trends in Cognitive Sciences, 4: 29-34.

Barrett, J. L. (2007) Cognitive Science of Religion: What Is It and Why Is It? Religion Compass, Vol 1(6): p. 768-786

Barrett, J. L. and Keil, F. C. (1996). Conceptualizing a nonnatural entity: Anthropomorphism in God concepts. Cognitive Psychology, 31:219-247 
Barsalou, L.W., Barbey, A.K., Simmons, W.K., \& Santos, A. (2005).

Embodiment in religious knowledge. Journal of Cognition and Culture, 5, 1457.

Bateson, G. (1973) Steps to an Ecology of Mind. London: Granada.

Bering, J. M. \& Johnson, D. D. P. (2005) "O Lord . . . you perceive my thoughts from afar": Recursiveness and the evolution of supernatural agency. Journal of Cognition and Culture 5:118-42.

Binford, L. (1962) Archaeology as anthropology. In Contemporary Archaeology, (ed.) by M. Leone, pp. 93-101. Southern Illinois University.

Boyd, R., and. Richerson. P. (1985) Culture and the Evolutionary Process. Chicago: University of Chicago Press.

Boyd, R. \& Richerson, P. (2002) Solving the puzzle of human cooperation. In: Levinson, S. (Ed.), Evolution and Culture. MIT Press, Cambridge, MA.

Boyer, P. (1994) The Naturalness of Religious Ideas: A Cognitive Theory of Religion, University of California Press, Berkeley, CA.

Boyer, P. (2001). Religion explained: The evolutionary origins of religious thought. Basic Books.

Boyer P. (2003) Religious thought and behaviour as by-products of brain function. Trends in Cognitive Sciences 7(3): p. 119-124.

Boyer, P. (2005) A reductionistic model of distinct modes of religious transmission. In Harvey Whitehouse \& Robert N. McCauley (eds.), Mind and religion: Psychological and cognitive foundations of religiosity, 3-29. Walnut Creek, CA: AltaMira Press.

Boyer, P. \& Ramble, C. (2001) Cognitive Templates for Religious Concepts: Crosscultural evidence for recall of counter-intuitive representations. Cognitive Science 25:535-64.

Boyer, P., \& Barrett, H.C. (2005) Evolved Intuitive Ontology: Integrating neural, behavioural and developmental aspects of domain-specificity. In Buss, D.M. (ed.). Handbook of evolutionary psychology. New York: Wiley

Bulbulia, J. (2004a) Religious Costs as Adaptations that Signal Altruistic Intention. Evolution and Cognition, 10 (1): 19-38.

Bulbulia, J. (2005) Are There Any Religions? An Evolutionary Exploration Method \& Theory in the Study of Religion, Volume 17, No 2: 71-100 
Bulbulia, J. (2006). Nature's medicine: Religiosity as an adaptation for health and cooperation. In P. McNamara (Ed.), Where God and science meet: Evolution, genes, and the religious brain (Vol. 1, pp. 87-121).

Bulbulia, J. (2007). Evolution and Religion. In R. I. Dunbar \& L. Barrett (Eds.), Oxford Handbook of Evolutionary Psychology (pp. 621-636). New York: Oxford University Press.

Bulbulia, J. (2008). Meme Infection or Religious Niche Construction? An Adapatationist Alternative to The Cultural Maladaptationist Hypothesis. Method and Theory in the Study of Religion, 20, 1-42.

Byrne, R. W. \& Whiten, A. (1988) Machiavellian Intelligence. Social Expertise and the Evolution of Intellect in Monkeys, Apes, and Humans, Oxford University Press, New York

Cajori, F. (1929) A History of Mathematical Notations, Volume II. Open Court Publishing Co., La Salle, Illinois.

Chase, P. G. (1994) On symbols and the palaeolithic. Current Anthropology, 35, 627-629.

Cheney, D. L. \& Seyfarth, R. M. (1992) Precis of How monkeys see the world. Behavioural and Brain Sciences 15:135-182.

Chomsky, N. (1955 / 1975) Logical Structure of Linguistic Theory. MIT Humanities Library. Microfilm. NewYork and London: Plenum Press

Chomsky, N. (1988) Language and Problems of Knowledge. Cambridge MA: MIT Press.

Churchland, P. (1989) Folk Psychology and the Explanation of Human Behaviour. In Churchland, A Neurocomputational Perspective. Cambridge, MA: MIT Press. Pp. 111-128.

Clark, A. (1996) Connectionism, Moral Cognition, and Collaborative Problem Solving, in Minds and Morals: Essays on Cognitive Science and Ethics, edited by Larry May, Marilyn Friedman and Andy Clark. Cambridge: MIT Press.

Clark, A. (1997) Being There: putting brain, body, and world together again. Cambridge, MA: MIT Press.

Clark, A. (1998) Where Brain, Body and World Collide Daedalus: Journal of the American Academy of Arts and Sciences (Special Issue on The Brain) Vol 127 (2) p. 257-280

Clark, A. (2001a) Mindware: An Introduction to the Philosophy of Cognitive Science. Oxford: Oxford University Press 
Clark, A. (2001b). Reasons, Robots, and the Extended Mind. Mind and Language 16, 121-145.

Clark, A. (2003) Natural-Born Cyborgs: minds, technologies, and the future of human intelligence. Oxford: Oxford U.P.

Clark, A. and Chalmers, D. (1998) The Extended Mind. Analysis 58, 7-19.

Clark A. and Grush, R. (1995) Towards a cognitive robotics. Adapt. Behav. 7: 5-16.

Cosmides, L. and Tooby, J. (1987) From Evolution to Behaviour: Evolutionary Psychology as the Missing Link, in J. Dupre, The Latest on the Best, Cambridge, MA, MIT Press, pp. 277-306.

Cosmides, L. and Tooby, J. (1994) Origins of Domain Specificity: the Evolution of Functional Organization, in L. Hirschfeld and S. Gelman (eds), Mapping the Mind, Cambridge: Cambridge University Press, pp. 85-116.

Cosmides, L., \& Tooby, J. (2000) Consider the source: The evolution of adaptations for decoupling and metarepresentation. In D. Sperber (Ed.), Metarepresentations: A multidisciplinary perspective (pp. 53-115). New York: Oxford University Press.

Dautenhahn, K. (1997) I could be you - The phenomenological dimension of social understanding. Cybernetics and Systems, 25(8), 417-453.

Dawkins, R. (1976) The Selfish Gene. Oxford: Oxford University Press.

Dawkins, R. (2003) A Devil's Chaplain. Weidenfeld and Nicolson, London.

Davies, M. \& Stone, T. (eds.) (1995) Folk Psychology. Oxford: Blackwell.

Day, M. (2004a) The Ins and Outs of Religious Cognition, Method and Theory in the Study of Religion. Vol. 16, 3: 241-255.

Day, M. (2004b) Religion, Off-Line Cognition and the Extended Mind, Journal of Cognition and Culture. Vol. 4, 1:101-121.

Day, M. (2005) Rethinking Naturalness: The Modes of Religiosity and Religion in the Round, In Harvey Whitehouse and Robert N. McCauley, eds., Mind and Religion: Psychological and Cognitive Foundations of Religion, 207-232. Walnut Creek, CA: Alta Mira Press.

Deacon, T. W. (1997) The symbolic species: The co-evolution of language and the brain. W. W. Norton.

Dennett, D. (1991). Consciousness Explained. Boston: Little, Brown and Co.

Dennett, D. (1996) Kinds of Minds. New York: Basic Books. 
Dennett, D. (2000) Making Tools for Thinking, in D. Sperber (ed.), Metarepresentations (Oxford U.P.).

Diamond, J. (2005) Collapse: How Societies Choose to Fail or Succeed. Viking, New York.

Donald, Merlin (1991). Origins of the Modern Mind. Cambridge, MA: Harvard U.P.

Donald, M. (1995) The neurobiology of human consciousness: an evolutionary approach. Neuropsychologia, 33:1087-1102.

Donald, Merlin (2001) A Mind So Rare: the evolution of human consciousness. New York: W.W. Norton.

Dunbar, R. (1996) Grooming, gossip and the evolution of language. Faber and Faber.

Durkheim, Emile (1963) [1915] Elementary Forms of the Religious Life. Joseph Swain, trans. London: Allen and Unwin.

Eliade, M. (1972) [1951] Shamanism: Archaic Techniques of Ecstasy.

Princeton: Princeton University Press.

Fodor, J. (1983) The Modularity of Mind. Cambridge, MA: Bradford Books / MIT Press.

Fuller R. C. (2007) Spirituality in the Flesh: The Role of Discrete Emotions in Religious Life. Journal of the American Academy of Religion, Vol. 75, No. 1, pp. 25-51

Galef, B. G. Jr (1996) Social influences on food preferences and feeding behaviours of vertebrates. In: Capaldi ED (ed) Why we eat what we eat. American Psychological Association, pp 207-231

Geertz, C. (1973) Thick Description: Toward an Interpretive Theory of Culture. In The Interpretation of Cultures. New York: Basic Books.

Geertz C.(2005) Shifting Aims, Moving Targets: On the Anthropology of Religion. Journal of the Royal Anthropological Institute, Vol. 11

Glucklich, A. (2001) Sacred Pain. New York: Oxford University Press.

Godfrey-Smith, P. (1996) Complexity and the Function of Mind in Nature. Cambridge University Press: New York.

Griffiths, P. and Gray R. (1994) "Developmental Systems and Evolutionary Explanation", Journal of Philosophy XCI: 277-304. 
Griffiths, P.E., Stotz, K. (2000) How the mind grows: a developmental perspective on the biology of cognition. Synthese 122, 29-51.

Guthrie. S. G. (1993) Faces in the clouds: A new theory of religion. Oxford University Press.

Hayden, B. (2002) Shamans, Sorcerers, and Saints. Washington, D.C.: Smithsonian Institution Books.

Hawkes C. (1954) Archaeological method and theory: some suggestions from the Old World. Am. Anthropol. 56:155.68.

Hutchins, E. (1995a) Cognition in the Wild. MIT Press

Hutchins, E. (1995b) How a cockpit remembers its speeds. Cognitive Science, 19, 265-288.

Irons, W. (1996) "Morality, Religion, and Human Nature." In Religion and Science: History, Method, Dialogue, ed. W. Mark Richardson and Wesley Wildman, 375-99. New York: Routledge.

Irons, W (2001). "Religion as a Hard-to-Fake Signal of Commitment."Chapter 13 in Randolph M. Ness, ed. Evolution and the Capacity for Commitment. New York, Russell Sage Foundations Series on Trust.

Jablonka, E. and Lamb, M. J. (2005). Evolution in Four Dimensions: Genetic, Epigenetic, Behavioural, and Symbolic Variation in the History of Life. Cambridge, MA: MIT Press.

James, W. (1900) Psychology (American Science Series, Briefer Course), New York: Henry Holt and Company.

Jeffares, B. (2002). The Scope and Limits of Biological Explanations in Archaeology. M.A. Philosophy. Wellington, Victoria University of Wellington.

Johnson M. and Rohrer, T. (2006) We Are Live Creatures: Embodiment, American Pragmatism, and the Cognitive Organism. In Body, Language, and Mind, v. 1, 17-54.

Karmiloff-Smith, A. (1992) Beyond modularity: A developmental perspective on cognitive science. MIT Press.

Keil, F. C. (1986) The Acquisition of Natural Kind and Artefact Terms. In Language Learning and Concept Acquisition. W. Demopoulos and A. Marras, eds. Pp. 133-153. Norwood, NJ: Ablex.

Keleman, D. (2004) Are children "intuitive theists"?: Reasoning about purpose and design in nature. Psychological Science 15:295-301. 
Krebs, J. R. \& Dawkins, R. (1984) Animal signals: mind-reading and manipulatiOt In Krebs, J. R. \& Davies, N. B. (eds.) Behavioural Ecology: An Evolutionary Approach. (2nd edition) Oxford: Blackwell Scientific Publications: 380-402.

Knight, C. (1998) Ritual/speech coevolution: A solution to the problem of deception. In: Approaches to the evolution of language, (ed.) J. R. Hurford, M. Studdert- Kennedy, \& C. Knight. Cambridge University Press.

Krippner, S. (2000) The Epistnuology and Technologies of Shamanic States of Consciousness. Journal of Consciousness Studies 7(11-12):93-118.

Laland, K. (2004) Extending the Extended Phenotype, Biology and Philosophy 19: $313-325$

Laland, K. \& Odling-Smee, J. (2000a) Niche Construction, Biological Evolution and Cultural Change. In Behavioural and Brain Sciences 23 (1): 131-175.

Laland, K. N. and Odling-Smee. J. (2000b) The evolution of the meme. In R. A. Aunger, (ed.), Darwinizing culture: The status of memetics as a science. Oxford: Oxford University Press.

Lawson, E.T. \& McCauley, R.N. (1990) Rethinking Religion: Connecting Cognition and Culture. Cambridge University Press

Leslie, A.M., (1987) Pretense and representation: the origins of "Theory of Mind". Psychological Review 94:412-426.

Leslie, A. M., \& Frith, U. (1987) Metarepresentation and autism: How not to lose one's marbles. Cognition, 27, 291-294.

Levy, G. (2005) Religious 'Cognition': Between Integrated Physiology and Network, Epoché: The University of California Journal for the Study of Religion 23:2, 193-224

Lewontin, R.C. (1982) Organism and environment. In Learning, Development, and Culture, H.C. Plotkin, (ed.), 151-170. New York: John Wiley \& Sons.

Lewontin, R. C., Rose, S., \& Kamin, L. J. (1984) Not in our genes. New York: Pantheon.

Mahoney, A. (2008). Theological Expressions as Costly Signals of Religious Commitment. In J. Bulbulia, R. Sosis, R. Genet, E. Harris, K. Wyman \& C. Genet (eds.), The Evolution of Religion: Studies, Theories, and Critiques. Santa Margarita, CA: Collins Foundation Press.

Malafouris L (2004), The Cognitive Basis of Material Engagement: Where Brain, Body and Culture Conflate, in Rethinking Materiality: The Engagement 
of Mind with the Material World, (eds.) E. DeMarrais, C. Gosden, C. Renfrew, Cambridge: McDonald Institute for Archaeological Research: 53-62

McCauley, R.N. (2000) The Naturalness of Religion and the Unnaturalness of Science, in F.C.Keil and R.A.Wilson (eds.), Explanation and Cognition, Cambridge University Press, Cambridge, pp. 61-85.

McBrearty, S. and Brooks, A. S. (2000) The revolution that wasn't: A new interpretation of the origin of modern human behaviour. Journal of Human Evolution 39:453-563.

Miller, E. K. (2000) The prefrontal cortex and cognitive control. Nature Rev. Neurol. 1: 59-65.

Mithen, S. (1996a) The Prehistory of the Mind: A Search for the Origins of Art, Religion, and Science. Thames \& Hudson.

Mithen, S. (1996b) The Supernatural Beings of Prehistory and the External Storage of Religious Ideas. In (eds.) Colin Renfrew and Christopher Scarre, Cognition and Material Culture: 97-106. Cambridge: The McDonald Institute for Archaeological Research, University of Cambridge.

Mithen, S. (2001) Archaeological Theory and Theories of Cognitive Evolution. In Hodder, I. (ed.), Archaeological Theory Today, Polity Press, Cambridge, pp. 98-121.

Newberg, A. B. and d'Aquili E. G. (2000). Neuropsychology of religious and spiritual experience. Journal of Consciousness Studies, 7 (11/12), pp. 251266.

Perner J, Leekam SR, Wimmer H. (1987) Three-year-olds. difficulty with false belief: the case for a conceptual deficit. Br. J. Dev. Psychol. 5:125.37

Pinker, S. (1997) How the Mind Works. New York, W. W. Norton \& Company.

Povinelli, D. J. (2000) Folk physics for apes: The chimpanzee's theory of how the world works. New York: Oxford University Press.

Pyysiäinen, I. (2004) Intuitive and Explicit in Religious Thought. Journal of Cognition and Culture 4(1):123-150

Pyysiäinen, I. (2006) Memories: Religion and Cultural Transmission. Anthropological Quarterly 79.2: 341-353.

Quartz, S.R. and Sejnowski, T. J. (1997) The Neural Basis of Cognitive Development: Constructivist Manifesto, Behavioural and Brain Sciences 20, 537-596.

Quartz, S.R.: 1999, The Constructivist Brain, Trends in Cognitive Sciences 3, 48-57. 
Renfrew, C. (2001) Symbol before concept: material engagement and the early development of society. In Hodder, I. (ed.), Archaeological Theory Today, Polity Press, Cambridge, 122-40.

Renfrew, C. (2008) The factuality of value and the sacred, Phil.Trans. R. Soc. B., 363: 2041-2047

Richerson, P.J. and Boyd, R. (1989) A Darwinian Theory for the Evolution of Symbolic Cultural Traits. In M. Freilich (ed.), The Relevance of Culture, 124147. Bergin and Garvey: Boston, MA

Richerson P.J. and Boyd, R. (2001) The evolution of subjective commitment to groups: A tribal instincts hypothesis. In: R.M. Nesse, (ed.), Evolution and the capacity for commitment. Russell Sage Foundation series on trust Vol. III, Russell Sage Foundation, New York, pp. 186-220.

Richerson, P.J. and Newson, L. (2008) Is religion adaptive? Yes, no, neutral, but mostly we don't know. In J. Bulbulia, R. Sosis, E. Harris, R. Genet, C. Genet \& K. Wyman (Eds.), The Evolution of Religion: Studies, Theories, and Critiques. Santa Margarita, CA: Collins Foundation Press.

Robb J. E. (1998) The archaeology of symbols. Annu. Rev. Anthropol. 27:329-46.

Ross, E. and Rapp, R. (1981) Sex and Society: A Research Note from Social History and Anthropology. Comparative Studies in Society and History 23:5172.

Samuels, R. (1998) Evolutionary psychology and the massive modularity hypothesis. The British Journal for the Philosophy Science 49: 575-602.

Savage-Rambaugh, S. and R. Lewin (1994) Kanzi, Doubleday, London.

Savage-Rambaugh, S. (1986) Ape Language: From Conditioned Response to Symbol, Oxford University Press, Oxford.

Schilling, T., \& Clifton, R. (1998) Nine-month-old infants learn about a physical event in a single session. Cognitive Development, 13, 165-184.

Schloss, J. (2008). He Who Laughs Best: Involuntary Religious Affect as a Solution to Recursive Cooperative Defection. In J. Bulbulia, R. Sosis, E. Harris, R. Genet, C. Genet \& K. Wyman (Eds.), The Evolution of Religion: Studies, Theories, and Critiques. Santa Margarita, CA: Collins Foundation Press.

Scholl B. J. and Leslie A. M. (1999) Modularity, development and 'theory of mind'. Mind and Language. 14:131-153. 
Shariff, A.F. \& Norenzayan, A. (2007). God is watching you: Priming God concepts increases prosocial behaviour in an anonymous economic game. Psychological Science, 18(9), 803-809.

Skyrms, B. (2003) The Stag Hunt and the Evolution of Social Structure. Cambridge Univ. Press, Cambridge, U.K.

Slone, D. J. (2004) Theological incorrectness: Why religious people believe what they shouldn't. Oxford University Press.

Sosis, R. (2000) Religion and intra-group cooperation: Preliminary results of acomparative analysis of utopian communities. Cross-Cultural Research 34:70-87.

Sosis, R., (2003) Why aren't we all Hutterites? Costly signaling theory and religious behaviour, Human Nature 14:91-127

Sosis, R. and C. Alcorta (2003) Signaling, solidarity and the sacred: the evolution of religious behaviour. Evolutionary Anthropology, 12: 264-274.

Sosis, R., Kress, H., \& Boster, J. (2007). Scars for war: Evaluating alternative explanations for cross-cultural variance in ritual costs. Unpublished manuscript, University of Connecticut, Storrs, CT.

Spelke, E. S. (1998) Nativism, empiricism, and the origins of knowledge. Infant Behaviour and Development, 21, 181-200. Wechsler, D. (1974).Wechsler

Spelke, E. S. (1990) Principles of Object Perception. Cognitive Science, 14: 29-56.

Sperber, D. (1985) Anthropology and Psychology: Towards an Epidemiology of Representations, Man, New Series, Vol. 20, No. 1. pp. 73-89.

Sperber, Dan. (1996) Explaining culture: A naturalistic approach. Oxford: Blackwell.

Sperber D. and Hirschfeld, L. A. (2004) The cognitive foundations of cultural stability and diversity. Trends in Cognitive Sciences, 8: 40-46.

Srejovic' D. (1969) Lepenski vir. Nova praistorijska kultura u Podunavlju. SKZ: Belgrade.

Stich, S. \& Nichols, S. (1992): Folk Psychology: Simulation or Tacit Theory?. Mind and Language, 7: 35-71

Sterelny, K. (2003). Thought in a hostile world: The evolution of human cognition. Oxford : Blackwell.

Sterelny, K. (2004) The Baldwin Effect and Its Significance: A Review of Bruce Weber and David Depew (eds) Evolution and Learning: The Baldwin Effect 
Reconsidered; MIT Press, Cambridge, Mass 2003, pp x, 341. To appear in: Evolution and Development.

Sterelny K. (2006) The evolution and evolvability of culture. Mind Lang. 21(2): 137-165.

Sterelny, K (2007) Social intelligence, human intelligence and niche construction. Philosophical Transactions of the Royal Society, Series B. Volume 362

Sterelny, K. (2008) SNAFUS, Biological Theory. Vol. 2, No. 3.

Teske, John A. (2001) The Genesis of Mind and Spirit. Zygon: Journal of Religion and Science, 36:93-104.

Tomasello, M. (1999) The Cultural Origins of Human Cognition. Cambridge, MA: Harvard University Press.

Tomasello, M., Savage-Rumbaugh, E. S. \& Kruger, A. C. (1993) Imitative learning of actions on objects by children, chimpanzees, and enculturated chimpanzees. Child Development 64:1688-1705.

Tooby J. and DeVore, I. (1987) The reconstruction of hominid behavioural evolution through strategic modeling. In: Primate Models of Hominid Behaviour, W. Kinzey (Ed.). New York: SUNY Press

Tooby, J. and Cosmides, L. (1992) The psychological foundations of culture. In J. Barkow, L. Cosmides, and J. Tooby (Eds.), The adapted mind: Evolutionary psychology and the generation of culture. New York: Oxford University Press.

Turner, J.S. (2000)The Extended Organism: The Physiology of Animal-Built Structures, Harvard University Press, Cambridge, MA.

Schjødt, U., Stødkilde-Jørgensen, H., Geertz, A., Roepstorff, A., (2008) Rewarding prayers. Neuroscience Letters, 443:165-168

White, R. (1993). Technological and social dimensions of "Aurignacian Age" body ornaments across Europe. In H. Knecht, A. Pike-Tay, and R. White (Eds.), Before Lascaux (pp. 277-99). Boca Raton, FL: CRC Press.

Whitehouse, H. (2004) Modes of Religiosity: A Cognitive Theory of Religious Transmission. Walnut Creek, CA: AltaMira Press.

Whitehouse, H. (2005) The Cognitive Foundations of Religiosity. In Harvey Whitehouse and Robert N. McCauley, eds., Mind and Religion: Psychological and Cognitive Foundations of Religion, 207-232. Walnut Creek, CA: AltaMira Press.

Wilson, D. S. (2002) Darwin's cathedral. University of Chicago Press. 
Young, F. (1962) The Function of Male Initiation Ceremonies: A Cross-Cultural Test of an Alternative Hypothesis. American Journal of Sociology 67:379-391.

Zahavi, A. (1987). The theory of signal selection and some of its implications. In Delfino, V. P. (ed.) International Symposium of Biological Evolution. Bari Adriatic Editrice: $305-327$. 